\begin{tabular}{|c|l|}
\hline Title & On stability of steady circular flows in a two-dimensional exterior disk \\
\hline Author(s) & Maekawa, Y asunori \\
\hline Citation & Hokkaido University Preprint Series in Mathematics, 1075, 1-75 \\
\hline Issue Date & 2015-8-27 \\
\hline DOI & 10.14943/84219 \\
\hline Doc URL & http:/hdl.handle.net/2115/69879 \\
\hline Type & bulletin (article) \\
\hline File Information & pre1075.pdf \\
\hline
\end{tabular}

Instructions for use 


\title{
On stability of steady circular flows in a two-dimensional exterior disk
}

\author{
Yasunori Maekawa
}

\begin{abstract}
We study the stability of some exact stationary solutions to the two-dimensional Navier-Stokes equations in an exterior domain to the unit disk. These stationary solutions are known as a simple model of the flow around a rotating obstacle, while their stability has been open due to the difficulty arising from their spatial decay in a scale-critical order. In this paper we affirmatively settle this problem for small solutions. That is, we will show that if these exact solutions are small enough then they are asymptotically stable with respect to small $L^{2}$ perturbations.
\end{abstract}

Keywords Navier-Stokes equations - two-dimensional exterior flows - scale-criticality · stability of stationary flows $\cdot$ flow around a rotating obstacle

Mathematics Subject Classification (2000) 35B35 - 35Q30 - 76D05 - 76D17

\section{Introduction}

In this paper we consider the two-dimensional Navier-Stokes equations for viscous incompressible flows

$$
\begin{cases}\partial_{t} u+u \cdot \nabla u=\Delta u-\nabla p, \quad \operatorname{div} u=0, & \text { for } x \in \Omega, \quad t>0, \\ u(x, t)=\alpha x^{\perp}, & \text { for } x \in \partial \Omega, \quad t>0, \\ u(x, 0)=u_{0}(x), & \text { for } x \in \Omega .\end{cases}
$$

Here $u=u(t, x)=\left(u_{1}(t, x), u_{2}(t, x)\right)$ and $p=p(t, x)$ are respectively unknown velocity field and pressure field, and $u_{0}(x)=\left(u_{0,1}(x), u_{0,2}(x)\right)$ is a given initial velocity field. We use the standard notation for derivatives: $\partial_{t}=\frac{\partial}{\partial t}, \partial_{j}=\frac{\partial}{\partial x_{j}}, \Delta=\sum_{j=1}^{2} \partial_{j}^{2}$, div $u=$ $\sum_{j=1}^{2} \partial_{j} u_{j}, u \cdot \nabla u=\sum_{j=1}^{2} u_{j} \partial_{j} u$. The domain $\Omega$ in $\mathbb{R}^{2}$ is assumed to be the exterior domain to the unit disk centered at the origin, i.e., $\Omega=\mathbb{R}^{2} \backslash \overline{B_{1}(0)}$ with $B_{r}(0)=\{x \in$ $\left.\mathbb{R}^{2}|| x \mid<r\right\}$, while $\alpha$ is a given real parameter and $x^{\perp}=\left(-x_{2}, x_{1}\right)$.

The initial-boundary value problem (NS) describes a simple model of the flow around a rotating obstacle. Indeed, the condition $u=\alpha x^{\perp}$ on $\partial \Omega$ represents the no-slip boundary condition of the flow around a rotating disk with a constant angular velocity $\alpha \in \mathbb{R}$, while the fluid domain $\Omega$ does not change under the rotation of the obstacle (disk) due to its radial symmetry. It is well known that (NS) has an explicit stationary solution

$$
\left(\alpha U, \alpha^{2} \nabla P\right) \quad \text { with } \quad U(x)=\frac{x^{\perp}}{|x|^{2}}, \quad P(x)=-\frac{1}{2|x|^{2}} .
$$

Yasunori Maekawa

Mathematical Institute, Tohoku University, 6-3, Aoba, Aramaki, Aoba, Sendai 980-8578, Japan

E-mail: maekawa@m.tohoku.ac.jp 
This exact solution represents a typical circular flow and provides a fundamental object in view of fluid dynamics in two dimensions. On the other hand, it has also an important aspect in view of nonlinear PDEs, for it is invariant under the scaling $U_{\lambda}(x)=\lambda U(\lambda x)$, $P_{\lambda}(x)=\lambda^{2} P(\lambda x), \lambda>0$, which is an invariant scaling for the stationary Navier-Stokes equations in the whole space. Hence, in a quantitative sense the solution (1) lies in the balance between the linearity (the Stokes part) and the nonlinearity (the convective part). It is one of the important themes in nonlinear PDEs to understand the stability property of global solutions possessing such a scale criticality. However, as is explained later, this stability problem is a difficult issue in general especially for the two-dimensional NavierStokes equations, due to the lack of the fundamental tool such as the Hardy-type inequality, which plays a central role in solving the problem in the higher dimensional cases. The aim of this paper is to study the $L^{2}$ stability of the special stationary solution (1), by which we reveal a typical structure of the flow around a rotating obstacle and also contribute to the analysis of the two-dimensional Navier-Stokes equations in a scale-critical framework.

In order to study the stability of the stationary flow (1) we consider the evolution of the perturbations $v=u-\alpha U$ and $p_{v}=p-\alpha^{2} P$, which should satisfy

$$
\partial_{t} v+v \cdot \nabla v=\Delta v-\alpha(U \cdot \nabla) v-\alpha(v \cdot \nabla) U-\nabla p_{v}, \quad \operatorname{div} v=0
$$

for $x \in \Omega$ and $t>0$, while

$$
v(x, t)=0 \quad \text { for } x \in \partial \Omega, t>0,\left.\quad v\right|_{t=0}=u_{0}-\alpha U \quad \text { for } x \in \Omega .
$$

We assume that the initial perturbation $v_{0}=u_{0}-\alpha U$ belongs to $L_{\sigma}^{2}(\Omega)$, where

$$
L_{\sigma}^{2}(\Omega)={\overline{C_{0, \sigma}^{\infty}(\Omega)}}^{\|\cdot\|_{L^{2}(\Omega)}}, \quad C_{0, \sigma}^{\infty}(\Omega)=\left\{f \in C_{0}^{\infty}(\Omega)^{2} \mid \operatorname{div} f=0 \quad \text { in } \Omega\right\} .
$$

Here $C_{0}^{\infty}(\Omega)$ is the set of smooth functions with compact support in $\Omega$. It is well known that the orthogonal projection $\mathbb{P}: L^{2}(\Omega)^{2} \rightarrow L_{\sigma}^{2}(\Omega)$, called the Helmholtz projection, is well-defined and satisfies $\mathbb{P} \nabla p=0$ for any $p \in L_{l o c}^{2}(\Omega)$ such that $\nabla p \in L^{2}(\Omega)^{2}$. Then the Stokes operator $A=-\mathbb{P} \Delta$ with the domain $D_{L_{\sigma}^{2}}(A)=W^{2,2}(\Omega)^{2} \cap W_{0}^{1,2}(\Omega)^{2} \cap L_{\sigma}^{2}(\Omega)$ defines a nonnegative self-adjoint operator in $L_{\sigma}^{2}(\Omega)$; cf. Sohr [44]. A standard way to analyze the system (2) - (3) is to rewrite it in the equivalent form

$$
\frac{\mathrm{d} v}{\mathrm{~d} t}+\mathbb{P}(v \cdot \nabla v)=-A_{\alpha} v, \quad t>0,\left.\quad v\right|_{t=0}=v_{0},
$$

where

$$
\begin{aligned}
D_{L_{\sigma}^{2}}\left(A_{\alpha}\right) & =D_{L_{\sigma}^{2}}(A), \\
A_{\alpha} v & =A v+\alpha \mathbb{P}(U \cdot \nabla v+v \cdot \nabla U), \quad v \in D_{L_{\sigma}^{2}}\left(A_{\alpha}\right) .
\end{aligned}
$$

Since $U$ is smooth and bounded in $\bar{\Omega}$ and $\mathbb{P}(U \cdot \nabla v+v \cdot \nabla U)$ is a lower order term, the standard perturbation theory of sectorial operators implies that the perturbed Stokes operator $-A_{\alpha}$ generates a $C_{0}$-analytic semigroup in $L_{\sigma}^{2}(\Omega)$ for any $\alpha \in \mathbb{R}$. Hence we convert $\left(\mathrm{NS}_{\alpha}\right)$ into the associated integral form

$$
v(t)=e^{-t A_{\alpha}} v_{0}-\int_{0}^{t} e^{-(t-s) A_{\alpha}} \mathbb{P}(v(s) \cdot \nabla v(s)) \mathrm{d} s .
$$

The solution of the integral equations $\left(\mathrm{INS}_{\alpha}\right)$ is called a mild solution to the Navier-Stokes equations (2) - (3). The main result is stated as follows. 
Theorem 1.1 For any sufficiently small $|\alpha|$ there exists a positive constant $\epsilon=\epsilon(\alpha)$ such that if $\left\|v_{0}\right\|_{L^{2}(\Omega)} \leq \epsilon$ then there exists a unique solution $v \in C\left([0, \infty) ; L_{\sigma}^{2}(\Omega)\right) \cap$ $C\left((0, \infty) ; W_{0}^{1,2}(\Omega)^{2}\right)$ to $\left(\mathrm{INS}_{\alpha}\right)$ satisfying $t^{1 / 2} \nabla v(t) \in L^{\infty}\left(0, \infty ; L^{2}(\Omega)\right)$ and

$$
\lim _{t \rightarrow \infty} t^{\frac{k}{2}}\left\|\nabla^{k} v(t)\right\|_{L^{2}(\Omega)}=0, \quad k=0,1 .
$$

Remark 1.2 When $\alpha=0$ the decay estimate (6) is well known; (6) with $k=0$ is proved by Masuda [37] and (6) with $k=1$ is proved by Kozono and Ogawa [30], without any restriction on the size of the initial data in $L_{\sigma}^{2}(\Omega)$. In this paper we are interested in the case $\alpha \neq 0$.

Theorem 1.1 shows that the stationary solution $\left(\alpha U, \alpha^{2} \nabla P\right)$ is asymptotically stable for small $L^{2}$ perturbations if $|\alpha|$ is sufficiently small. In order to prove the nonlinear stability as in Theorem 1.1, we need to establish several estimates for the perturbed Stokes semigroup $\left\{e^{-t A_{\alpha}}\right\}_{t \geq 0}$. The next theorem serves our purpose.

Theorem 1.3 There exists a positive constant $\delta$ such that if $|\alpha| \leq \delta$ then the following statement holds. Let $1<q \leq 2 \leq p<\infty$. Then it follows that

$$
\begin{aligned}
& \left\|e^{-t A_{\alpha}} f\right\|_{L^{p}(\Omega)} \leq C t^{-\frac{1}{q}+\frac{1}{p}}\|f\|_{L^{q}(\Omega)}, \quad t>0, \\
& \left\|\nabla e^{-t A_{\alpha}} f\right\|_{L^{2}(\Omega)} \leq C t^{-\frac{1}{q}}\|f\|_{L^{q}(\Omega)}, \quad t>0,
\end{aligned}
$$

for $f \in L_{\sigma}^{2}(\Omega) \cap L^{q}(\Omega)^{2}$. Here the constant $C$ depends only on $\alpha$, $p$, and $q$.

Remark 1.4 (1) Set $L_{\sigma}^{q}(\Omega)={\overline{C_{0, \sigma}^{\infty}(\Omega)}}^{\|\cdot\|_{L^{q}(\Omega)}}, 1 \leq q<\infty$. Then, by the density argument the estimates (7) and (8) hold for all $f \in L_{\sigma}^{q}(\Omega), 1<q \leq 2$.

(2) The $L^{p}-L^{q}$ estimates for the Stokes semigroup $\left\{e^{-t A}\right\}_{t \geq 0}$ (i.e., $\alpha=0$ ) in twodimensional exterior domains are well known; see Maremonti and Solonnikov [36] and Dan and Shibata [4,5]. We will focus on the case $\alpha \neq 0$ in this paper. In fact, the lower order term of $A_{\alpha}$ in (5) leads to the disappearance of a logarithmic singularity for the resolvent kernel which is present in the case $\alpha=0$. This is considered as a stabilizing effect due to the rotation of the obstacle. We will essentially use this property to obtain the resolvent estimates in Section 3.3. As a result, the constant $C$ in (7) and (8) obtained so far is highly depending on $\alpha \neq 0$ and tends to $\infty$ as $\alpha \rightarrow 0$, although (7) and (8) are known to hold for the limit case $\alpha=0$.

Here let us briefly recall some works related to our results.

(i) Existence and stability of stationary solutions with decay order $O\left(|x|^{-1}\right)$

In the case of three-dimensional (fixed) exterior domains the unique existence of stationary Navier-Stokes flows satisfying the decay estimate $O\left(|x|^{-1}\right)$ (for velocity) as $|x| \rightarrow \infty$ is proved by Finn [12], Galdi-Simader [16], Novotny and Padula [39], and Borchers and Miyakawa [3] under some smallness and decay conditions on given data, and in Korolev and Šverák [29] the asymptotic profile at spatial infinity is shown to be the Landau solution. The existence theory in the Lorenz spaces has been established by Kozono and Yamazaki [33]. The local $L^{r}$ stability of these stationary solutions is established in [3] and Kozono and Yamazaki [32], while the global $L^{2}$ stability is shown in [3]. The reader is referred to recent results by Karch, Pilarczyk, and Schonbek [28] and Hishida and Schonbek [24], where the global $L^{2}$ stability of small global solutions in $L^{\infty}\left(0, \infty ; L_{\sigma}^{3, \infty}\left(\mathbb{R}^{3}\right)\right)$ is obtained. 
When $\Omega \subset \mathbb{R}^{n}$ is an $n$-dimensional exterior domain we also recall that the unique existence of small global solutions in $L^{\infty}\left(0, \infty ; L_{\sigma}^{n, \infty}(\Omega)\right)$ for given small initial data in $L_{\sigma}^{n, \infty}(\Omega)$ is proved by Kozono and Yamzaki [31] for $n \geq 2$, while its local stability in the framework of the Lorentz space is achieved for the case $n \geq 3$ by Yamazaki [45]. Contrary to the higher dimensional cases, less is known so far for the case of two-dimensional exterior domains. Indeed, for the two-dimensional exterior problem the unique existence of stationary flows decaying at spatial infinity has been achieved mainly under some symmetry conditions on both the domain and given data; see Galdi [14], Russo [41], Yamazaki [46], and Pileckas and Russo [40], and see also Nakatsuka [38] for a recent uniqueness result. In particular, Yamazaki [46] obtained the decay order $O\left(|x|^{-1}\right)$ for the stationary solutions constructed there. Recently, Hillairet and Wittwer [19] succeeded in constructing stationary solutions around the exact solution (1) when the domain is an exterior disk and the angular velocity $|\alpha|$ is large. Note that the stationary solution obtained in [19] asymptotically behaves like $\alpha U(x)$ as $|x| \rightarrow \infty$. The argument in [19] is based on the streamfunction-vorticity formulation in the polar coordinates, and our approach in this paper is motivated by their work. In the two-dimensional case the stability of stationary flows mentioned above is widely open, even in the case when both the stationary flows and the initial perturbations are small enough. Related to the stability of scale-critical flows in two-dimensional exterior domains, we note that the global asymptotic stability of small time-dependent solutions in [31], which may behave like forward self-similar solutions, has recently established by the present author [35]. The result of [35] is an extension of the global stability result by Gallay and the author [17] for the Lamb-Oseen vortex in two-dimensional exterior domains; see also Iftimie, Karch, and Lacave [25, 26] for a recent stability result of time-dependent global solutions. The approach used in $[25,17,26,35]$, however, highly relies on a scale-critical temporal decay of small global solutions, and therefore, it is not applied to the stability problem for stationary solutions. As far as the author knows, Theorem 1.1 is the first stability result for the two-dimensional stationary flows decaying in the scale-critical order $O\left(|x|^{-1}\right)$.

(ii) Mathematical analysis of the flow around a rotating obstacle

So far mathematical results have been obtained mainly for the three-dimensional problem, as stated below. As for the nonstationary problem, the existence of global weak solutions is proved by Borchers [2], and the unique existence of time-local regular solutions is shown by Hishida [20] and Geissert, Heck, and Hieber [18], while the global strong solutions for small data are obtained by Galdi and Silvestre [15]. The spectrum of the linear operator arising in this problem is studied by Farwig and Neustupa [10]; see also the linear analysis by Hishida [21]. The existence of steady-state solutions to the associated system is proved in [2], Silvestre [42], Galdi [13], and Farwig and Hishida [7]. In particular, in [13] the steady flows with the decay order $O\left(|x|^{-1}\right)$ are obtained. On th other hand, the asymptotic profile of these steady flows at spatial infinity is studied by Farwig and Hishida [8,9] and Farwig, Galdi, and Kyed [6]. The stability of these steady solutions is proved in [15] and Hishida and Shibata [23] in the $L^{2}$ and $L^{q}$ functional framework, respectively. All results mentioned above are in the three-dimensional case, and there are still few results for the flow around a rotating obstacle in the two-dimensional case. The reader is referred to a recent work by Hishida [22], where the asymptotic behavior of the two-dimensional steady Stokes flow around a rotating obstacle is investigated in details, and it is shown there that the leading profile is given by a constant multiple of the circular flow (1).

The crucial difficulty in obtaining Theorems 1.1 and 1.3 is that the following Hardy 
inequality is not available in the two-dimensional case:

$$
\left\|\frac{f}{|x|}\right\|_{L^{2}(\Omega)} \leq C\|\nabla f\|_{L^{2}(\Omega)}, \quad f \in \dot{W}_{0}^{1,2}(\Omega)^{2} .
$$

The inequality (9) is valid if $\Omega$ is an exterior domain in $\mathbb{R}^{n}$ with $n \geq 3$, and it is the most essential tool in showing the stability of stationary solutions decaying in the scalecritical order $O\left(|x|^{-1}\right)$ as $|x| \rightarrow \infty$ when the dimension $n$ is higher than or equal to three. When $\Omega$ is an exterior domain in $\mathbb{R}^{2}$ the Hardy inequality of the form (9) does not hold unless one imposes some symmetry on both $\Omega$ and $f$, see $[14,38]$, and the absence of the Hardy inequality (9) has been a crucial obstacle to achieve the stability result for stationary solutions decaying like $O\left(|x|^{-1}\right)$, even in the case when both the stationary solution and the perturbation are small enough. We note that, although the domain $\Omega$ is assumed to be exterior to the unit disk in this paper, there is no symmetry condition on the perturbations in Theorem 1.1. In particular, the approach based on the Hardy inequality (9) is not applicable to our problem, and we need to overcome this essential difficulty.

The key step in the proof of Theorems 1.1 and 1.3 is to study the spectral property of the perturbed Stokes operator $A_{\alpha}$ by focusing on the rotational symmetry of the domain and the circular flow (1). As will be seen below, the analysis using the streamfunction-vorticity formulation in the polar coordinates reveals some crucial informations on the spectrum of $A_{\alpha}$ in $L_{\sigma}^{2}(\Omega)$ even when $|\alpha|$ is not necessarily small. To state the result let us introduce the modified Bessel function of second kind of order $\mu$, denoted by $K_{\mu}$, which is defined as

$$
K_{\mu}(z)=\frac{1}{2} \int_{0}^{\infty} e^{-\frac{z}{2}\left(t+\frac{1}{t}\right)} t^{-\mu-1} \mathrm{~d} t, \quad \Re(z)>0 .
$$

Next we set

$$
\mu_{n}(\alpha)=\left(n^{2}+i \alpha n\right)^{\frac{1}{2}}, \quad n \in \mathbb{Z} \backslash\{0\},
$$

where the square root is taken so that $\Re\left(\mu_{n}(\alpha)\right)>0$, and we also set

$$
F_{n}(z ; \alpha)=\int_{1}^{\infty} s^{1-|n|} K_{\mu_{n}(\alpha)}(s z) \mathrm{d} s, \quad \Re(z)>0, \quad n \in \mathbb{Z} \backslash\{0\} .
$$

We denote by $\sigma\left(-A_{\alpha}\right)$ the spectrum of $-A_{\alpha}$ in $L_{\sigma}^{2}(\Omega)$, and by $\sigma_{\text {disc }}\left(-A_{\alpha}\right)$ the set of discrete eigenvalues of $-A_{\alpha}$ with finite algebraic multiplicity. We set $\mathbb{R}_{-}=\{x \in \mathbb{R} \mid x<$ $0\}$ and $\Sigma_{\phi}=\{z \in \mathbb{C} \backslash\{0\}|| \arg (z) \mid<\phi\}$. We use the standard notation for the polar coordinates: $x_{1}=r \cos \theta, x_{2}=r \sin \theta, r=|x|$, and $\theta \in[0,2 \pi)$. For $z \in \mathbb{C} \backslash \overline{\mathbb{R}_{-}}$its square root $\sqrt{z}$ is always taken so that $\Re(\sqrt{z})>0$.

Theorem 1.5 Let $\alpha \in \mathbb{R}$. Then the following statements hold.

(1) $\sigma\left(-A_{\alpha}\right)=\overline{\mathbb{R}_{-}} \cup \sigma_{\text {disc }}\left(-A_{\alpha}\right)$ and

$$
\sigma_{\text {disc }}\left(-A_{\alpha}\right)=\left\{\lambda \in \mathbb{C} \backslash \overline{\mathbb{R}_{-}} \mid F_{n}(\sqrt{\lambda} ; \alpha)=0 \text { for some } n \in \mathbb{Z} \backslash\{0\}\right\} .
$$

(2) The eigenspace $E_{\alpha}(\lambda)$ for the eigenvalue $\lambda \in \sigma_{\text {disc }}\left(-A_{\alpha}\right)$ is spanned by $\left\{V_{n, \lambda, \alpha}\right\}_{n \in Z_{\alpha}(\lambda)}$, where $Z_{\alpha}(\lambda)=\left\{n \in \mathbb{Z} \backslash\{0\} \mid F_{n}(\sqrt{\lambda} ; \alpha)=0\right\}$ and $V_{n, \lambda, \alpha}$ is the velocity field whose vorticity is given as $K_{\mu_{n}(\alpha)}(\sqrt{\lambda} r) e^{i n \theta}$ in the polar coordinates. 
(3) Each $\lambda_{0} \in \sigma_{\text {disc }}\left(-A_{\alpha}\right)$ is a pole of the resolvent $\left(\lambda+A_{\alpha}\right)^{-1}$ of order at most two. Moreover, if $\lambda_{0} \in \sigma_{\text {disc }}\left(-A_{\alpha}\right)$ belongs to the set

$$
\{z \in \mathbb{C}|| \Im(z)|\geq-| \alpha \mid \Re(z)\},
$$

then $\lambda_{0}$ is a pole of $\left(\lambda+A_{\alpha}\right)^{-1}$ of order one, that is, it is a semisimple eigenvalue with multiplicity $\sharp Z_{\alpha}\left(\lambda_{0}\right)$.

(4) For any $\epsilon \in\left(0, \frac{\pi}{2}\right)$ there exists a positive constant $\delta_{\epsilon}$ such that if $|\alpha| \leq \delta_{\epsilon}$ then the sector $\Sigma_{\pi-\epsilon}$ is included in the resolvent set $\rho\left(-A_{\alpha}\right)$.

In the statement (1) of Theorem 1.5 the structure of the spectrum $\sigma\left(-A_{\alpha}\right)=\overline{\mathbb{R}_{-}} \cup$ $\sigma_{\text {disc }}\left(-A_{\alpha}\right)$ itself is a result from the general perturbation theory of linear operators, for $A_{\alpha}$ is a relatively compact perturbation of the Stokes operator $A$ which is nonnegative selfadjoint in $L_{\sigma}^{2}(\Omega)$; see Section 2.4. What is striking here is the characterization (13) of the discrete spectrum as the set of zero points of $F_{n}(\sqrt{\lambda} ; \alpha)$, which is defined as (12) and is analytic in $\mathbb{C} \backslash \overline{\mathbb{R}_{-}}$. In general it is not easy to know the distribution of zero points of $F_{n}(\sqrt{\lambda} ; \alpha)$. Nevertheless, it is possible to determine the eigenspace for each discrete eigenvalue, as is shown in (2) and (3) of Theorem 1.5. The precise definition of the velocity $V_{n, \lambda, \alpha}$ in (2) is stated in Proposition 3.2. As is observed from the representation of the resolvent in Proposition 3.4, the order of poles of $\left(\lambda+A_{\alpha}\right)^{-1}$ in $\mathbb{C} \backslash \overline{\mathbb{R}_{-}}$is related to the order of zero points of $F_{n}(\sqrt{\lambda} ; \alpha)$, which is the key to prove (3). We note that the statements (1), (2), and (3) of Theorem 1.5, which are proved in Sections 3.1 and 3.2, hold without any restriction on the size of $\alpha$, while (4) is obtained so far only in the case of small $|\alpha|$ even when $\epsilon$ is close to $\frac{\pi}{2}$.

Since the continuous spectrum of $-A_{\alpha}$ lies on $\overline{\mathbb{R}_{-}}$, the estimate of $\left\{e^{-t A_{\alpha}}\right\}_{t \geq 0}$ in Theorem 1.3 is not trivial even if the spectral stability as in Theorem 1.5 (4) is proved, and one needs to study the behavior of the resolvent $\left(\lambda+A_{\alpha}\right)^{-1}$ for $|\lambda| \ll 1$. In fact, we will observe in Section 3.5 that the estimate of the resolvent near $\lambda=0$ can be obtained for any size of $\alpha$. As a result, the spectral stability does lead to the estimates of $\left\{e^{-t A_{\alpha}}\right\}_{t \geq 0}$, and Theorem 1.3 can be described in a more general form as follows. We denote by $\bar{\Sigma}_{\phi}$ the closure of the set $\Sigma_{\phi}$ below.

Theorem 1.6 Let $\alpha \in \mathbb{R} \backslash\{0\}$. Let $m_{\alpha}$ be the integer satisfying $|\alpha|<m_{\alpha} \leq|\alpha|+1$ and set $\theta_{\alpha} \in\left(0, \frac{\pi}{2}\right)$ as $\theta_{\alpha}=\operatorname{Arctan} \frac{|\alpha|}{m_{\alpha}-|\alpha|}$. Assume that there is $\epsilon_{0} \in\left(\theta_{\alpha}, \frac{\pi}{2}\right)$ such that

$$
F_{n}(\sqrt{\lambda} ; \alpha) \neq 0 \quad \text { for all } \lambda \in \bar{\Sigma}_{\pi-\epsilon_{0}} \backslash\{0\} \text { and } 1 \leq|n| \leq m_{\alpha} .
$$

Then $\Sigma_{\pi-\epsilon_{0}} \subset \rho\left(-A_{\alpha}\right)$ and the following resolvent estimate holds.

$$
\left\|\left(\lambda+A_{\alpha}\right)^{-1}\right\|_{\mathcal{L}\left(L_{\sigma}^{2}\right)} \leq \frac{C}{|\lambda|} \quad \text { for } \lambda \in \Sigma_{\pi-\epsilon_{0}} .
$$

Here the constant $C$ depends only on $\alpha$ and $\epsilon_{0}$. Moreover, the perturbed Stokes semigroup $\left\{e^{-t A_{\alpha}}\right\}_{t \geq 0}$ satisfies the estimates (7) and (8) for $1<q \leq 2 \leq p<\infty$ with a constant $C$ depending only on $\alpha, p, q$, and $\epsilon_{0}$.

Remark 1.7 (1) Once we have obtained the estimates (7) and (8) for $\left\{e^{-t A_{\alpha}}\right\}_{t \geq 0}$ then it is a routine work to show the nonlinear stability as in Theorem 1.1. Hence, the absence of zero points of $F_{n}(\sqrt{\lambda} ; \alpha)$ as in (15), if it holds, also implies the nonlinear stability of $\alpha U$ with respect to small $L^{2}$ perturbations. 
(2) Let $\epsilon \in\left(0, \frac{\pi}{2}\right)$. It is shown that for any $\alpha \in \mathbb{R}$ there is $r_{\alpha, \epsilon}>0$ such that

$$
\Sigma_{\pi-\epsilon} \cap B_{r_{\alpha, \epsilon}}(0) \subset \rho\left(-A_{\alpha}\right),
$$

see Remark 3.30. Hence, even if the linear instability occurs for some $\alpha$, the curve of the eigenvalue $\lambda(\alpha)$ satisfying $\Re(\lambda(\alpha))=\sup \left\{\Re(\lambda) \mid \lambda \in \sigma_{\text {disc }}\left(-A_{\alpha}\right)\right\}$ never crosses the origin.

Theorem 1.6 enables us to reduce the analysis of the perturbed Stokes operator and the associated semigroup to the study of zero points of the analytic function $F_{n}(z ; \alpha)$ in some sector for a finite number of $n$. This is our key strategy to prove Theorem 1.3. Indeed, in Section 3.6 we will show that for any $\epsilon \in\left(0, \frac{\pi}{2}\right)$ if $|\alpha|$ is sufficiently small then there are no zero points of $F_{n}(\sqrt{\lambda} ; \alpha)$ with $|n|=1$ in the sector $\Sigma_{\pi-\epsilon}$. Theorem 1.3 and the statement (4) of Theorem 1.5 follow from this result and Theorem 1.6. Even for the case $|\alpha| \ll 1$ the nonexistence of zero points of $F_{n}(\sqrt{\lambda} ; \alpha)$ is in fact highly nontrivial, and we need to use the asymptotic estimates of the modified Bessel functions and several identities between special functions.

This paper is organized as follows. In Section 2.1 we collect some basic results on vector fields in the polar coordinates. In particular, the orthogonal projections associated to the Fourier modes with respect to the angular variable are introduced. Then the Biot-Savart law in each Fourier mode is recalled in Section 2.2. The invariant property of the perturbed Stokes operator $A_{\alpha}$ is stated in Section 2.3, which leads to a decomposition of $A_{\alpha}$ into the operators in each Fourier mode. In Section 2.4 we show that $A_{\alpha}$ is a relatively compact perturbation of the Stokes operator in $L_{\sigma}^{2}(\Omega)$. Section 3 is a core of this paper, which is devoted to the spectral analysis (Section 3.1) and the resolvent analysis (Sections 3.2 and 3.3) of $A_{\alpha}$. In Section 3.1 we give a characterization of the discrete spectrum of $A_{\alpha}$, while the key structure of the resolvent is shown in Section 3.2 (see Proposition 3.4). The detailed resolvent estimates are obtained in Section 3.3, where we need a long computation based on the asymptotic estimates of the modified Bessel functions which are collected in the appendix. The main results in Section 3.3 are Theorems 3.19 and 3.23. In Section 3.4 we establish some resolvent estimates but with the standard energy method. Then, collecting the results obtained in the previous sections, we prove Theorem 1.6 in Section 3.5. Section 3.6 is devoted to the linear analysis for the case $|\alpha| \ll 1$, where the main goal is to show the nonexistence of zero points of $F_{n}(\sqrt{\lambda} ; \alpha)$ with $|n|=1$ in a suitable sector. The nonlinear problem is then considered in Section 4, and Theorem 1.1 is proved in this final section.

\section{Preliminaries}

In order to show Theorem 1.5 we analyze the perturbed Stokes operator $A_{\alpha}$ in the polar coordinates

$$
x_{1}=r \cos \theta, \quad x_{2}=r \sin \theta, \quad r=|x| \geq 1, \quad \theta \in[0,2 \pi) .
$$

In this section we state some basic results on the vector fields in the polar coordinates. 


\subsection{Vector fields in the polar coordinates}

We set

$$
\mathbf{e}_{r}=\frac{x}{|x|}, \quad \mathbf{e}_{\theta}=\frac{x^{\perp}}{|x|}=\partial_{\theta} \mathbf{e}_{r}
$$

For $v \in L^{2}(\Omega)^{2}$ we set $v_{r}=v \cdot \mathbf{e}_{r}$ and $v_{\theta}=v \cdot \mathbf{e}_{\theta}$, and thus $v=v_{r} \mathbf{e}_{r}+v_{\theta} \mathbf{e}_{\theta}$. The operators div and rot in the polar coordinates are described as follows.

Lemma 2.1 Let $v \in W^{1,2}(\Omega)^{2}$. Then it follows that

$$
\begin{aligned}
\operatorname{div} v & =\partial_{1} u_{1}+\partial_{2} u_{2}=\frac{1}{r} \partial_{r}\left(r v_{r}\right)+\frac{1}{r} \partial_{\theta} v_{\theta}, \\
\operatorname{rot} v & =\partial_{1} u_{2}-\partial_{2} u_{1}=\frac{1}{r} \partial_{r}\left(r v_{\theta}\right)-\frac{1}{r} \partial_{\theta} v_{r}, \\
|\nabla v|^{2} & =\left|\partial_{r} v_{r}\right|^{2}+\left|\partial_{r} v_{\theta}\right|^{2}+\frac{1}{r^{2}}\left(\left|\partial_{\theta} v_{r}-v_{\theta}\right|^{2}+\left|v_{r}+\partial_{\theta} v_{\theta}\right|^{2}\right) .
\end{aligned}
$$

Proof. The identities (18) and (19) are straightforward and we omit the proof. The identity (20) follows from

$$
\begin{aligned}
\partial_{1} v & =\cos \theta \partial_{r}\left(v_{r} \mathbf{e}_{r}+v_{\theta} \mathbf{e}_{\theta}\right)-\frac{\sin \theta}{r} \partial_{\theta}\left(v_{r} \mathbf{e}_{r}+v_{\theta} \mathbf{e}_{\theta}\right) \\
& =\left(\partial_{r} v_{r} \cos \theta-\left(\partial_{\theta} v_{r}-v_{\theta}\right) \frac{\sin \theta}{r}\right) \mathbf{e}_{r}+\left(\partial_{r} v_{\theta} \cos \theta-\left(\partial_{\theta} v_{\theta}+v_{r}\right) \frac{\sin \theta}{r}\right) \mathbf{e}_{\theta}
\end{aligned}
$$

and

$$
\begin{aligned}
\partial_{2} v & =\sin \theta \partial_{r}\left(v_{r} \mathbf{e}_{r}+v_{\theta} \mathbf{e}_{\theta}\right)+\frac{\cos \theta}{r} \partial_{\theta}\left(v_{r} \mathbf{e}_{r}+v_{\theta} \mathbf{e}_{\theta}\right) \\
& =\left(\partial_{r} v_{r} \sin \theta+\left(\partial_{\theta} v_{r}-v_{\theta}\right) \frac{\cos \theta}{r}\right) \mathbf{e}_{r}+\left(\partial_{r} v_{\theta} \sin \theta+\left(\partial_{\theta} v_{\theta}+v_{r}\right) \frac{\cos \theta}{r}\right) \mathbf{e}_{\theta}
\end{aligned}
$$

The details are omitted here. The proof is complete.

For each $n \in \mathbb{Z}$ we denote by $\mathcal{P}_{n}$ the projection on the Fourier mode $n$ with respect to the angular variable $\theta$, that is,

$$
\mathcal{P}_{n} v=v_{r, n} e^{i n \theta} \mathbf{e}_{r}+v_{\theta, n} e^{i n \theta} \mathbf{e}_{\theta},
$$

where

$$
\begin{aligned}
& v_{r, n}(r)=\frac{1}{2 \pi} \int_{0}^{2 \pi} v_{r}(r \cos \theta, r \sin \theta) e^{-i n \theta} \mathrm{d} \theta, \\
& v_{\theta, n}(r)=\frac{1}{2 \pi} \int_{0}^{2 \pi} v_{\theta}(r \cos \theta, r \sin \theta) e^{-i n \theta} \mathrm{d} \theta .
\end{aligned}
$$

Next we set for $m \in \mathbb{N} \cup\{0\}$,

$$
\mathcal{Q}_{m} v=\sum_{|n|=m+1}^{\infty} \mathcal{P}_{n} v
$$


It is easy to see that the right-hand side of (22) converges in $L^{2}(\Omega)^{2}$ and each of $\mathcal{P}_{n}, \mathcal{Q}_{m}$ : $L^{2}(\Omega)^{2} \rightarrow L^{2}(\Omega)^{2}$ is an orthogonal projection. Moreover, the divergence free condition as well as the no-flux condition $\mathbf{e}_{r} \cdot v=0$ on the boundary $\partial \Omega$ is preserved under the action of $\mathcal{P}_{n}$. More precisely, we have

$$
\begin{aligned}
\mathcal{P}_{n} C_{0, \sigma}^{\infty}(\Omega) \subset C_{0, \sigma}^{\infty}(\Omega), & & \mathcal{Q}_{m} C_{0, \sigma}^{\infty}(\Omega) \subset C_{0, \sigma}^{\infty}(\Omega), \\
\mathcal{P}_{n} \mathbb{P}=\mathbb{P P}_{n}, & & \mathcal{Q}_{m} \mathbb{P}=\mathbb{P} \mathcal{Q}_{m} .
\end{aligned}
$$

Therefore, for each $m \in \mathbb{N} \cup\{0\}$ we have an orthogonal decomposition of $L_{\sigma}^{2}(\Omega)$ as

$$
L_{\sigma}^{2}(\Omega)=L_{\sigma, r a d}^{2}(\Omega) \oplus \mathcal{Q}_{m} L_{\sigma}^{2}(\Omega) \oplus\left(\oplus_{1 \leq|n| \leq m} \mathcal{P}_{n} L_{\sigma}^{2}(\Omega)\right),
$$

where $L_{\sigma, \text { rad }}^{2}(\Omega)=\mathcal{P}_{0} L_{\sigma}^{2}(\Omega)$ is the space of radially symmetric flows in $L_{\sigma}^{2}(\Omega)$.

Next we recall the action of the Laplace operator $-\Delta$ for $v \in W^{2,2}(\Omega)^{2}$ in the polar coordinates. The direct calculation using $v=v_{r} \mathbf{e}_{r}+v_{\theta} \mathbf{e}_{\theta}$ shows that

$$
\begin{aligned}
-\Delta v= & \left(-\partial_{r}\left(\frac{1}{r} \partial_{r}\left(r v_{r}\right)\right)-\frac{1}{r^{2}} \partial_{\theta}^{2} v_{r}+\frac{2}{r^{2}} \partial_{\theta} v_{\theta}\right) \mathbf{e}_{r} \\
& +\left(-\partial_{r}\left(\frac{1}{r} \partial_{r}\left(\left(r v_{\theta}\right)\right)-\frac{1}{r^{2}} \partial_{\theta}^{2} v_{\theta}-\frac{2}{r^{2}} \partial_{\theta} v_{r}\right) \mathbf{e}_{\theta} .\right.
\end{aligned}
$$

Hence we have an invariant relation

$$
\mathcal{P}_{n} \Delta \subset \Delta \mathcal{P}_{n}, \quad \mathcal{Q}_{m} \Delta \subset \Delta \mathcal{Q}_{m}
$$

From (23), (24), and (26) the Stokes operator $A=-\mathbb{P} \Delta$ is diagonalized as

$$
A=\left.\left.A\right|_{L_{\sigma, \text { rad }}^{2}} \oplus A\right|_{\mathcal{Q}_{m} L_{\sigma}^{2}} \oplus\left(\left.\oplus_{1 \leq|n| \leq m} A\right|_{\mathcal{P}_{n} L_{\sigma}^{2}}\right) .
$$

Here, for an invariant subspace $X \subset L_{\sigma}^{2}(\Omega)$ the operator $\left.A\right|_{X}$ is defined as $D\left(\left.A\right|_{X}\right)=$ $D_{L_{\sigma}^{2}}(A) \cap X$ and $\left.A\right|_{X}: D\left(\left.A\right|_{X}\right) \rightarrow X,\left.A\right|_{X} u=A u$ for $u \in D\left(\left.A\right|_{X}\right)$. In Section 2.3 we will see that, due to the radial symmetry of $U$, the perturbed Stokes operator $A_{\alpha}$ is also diagonalized as in (27).

The following lemma is a direct consequence of Lemma 2.1 and the definition of $\mathcal{P}_{n}$.

Lemma 2.2 Let $n \in \mathbb{N} \cup\{0\}$. For any $v \in W^{1,2}(\Omega)^{2}$ it follows that

$$
\begin{aligned}
\|\nabla v\|_{L^{2}(\Omega)}^{2} & =\sum_{n \in \mathbb{Z}}\left\|\nabla \mathcal{P}_{n} v\right\|_{L^{2}(\Omega)}^{2}, \\
\left|\nabla \mathcal{P}_{n} v\right|^{2} & =\left|\partial_{r} v_{r, n}\right|^{2}+\frac{1+n^{2}}{r^{2}}\left|v_{r, n}\right|^{2}+\left|\partial_{r} v_{\theta, n}\right|^{2}+\frac{1+n^{2}}{r^{2}}\left|v_{\theta, n}\right|^{2}-\frac{4 n}{r^{2}} \Im\left(v_{\theta, n} \overline{v_{r, n}}\right) .
\end{aligned}
$$

In particular, we have

$$
\left|\nabla \mathcal{P}_{n} v\right|^{2} \geq\left|\partial_{r} v_{r, n}\right|^{2}+\frac{(|n|-1)^{2}}{r^{2}}\left|v_{r, n}\right|^{2}+\left|\partial_{r} v_{\theta, n}\right|^{2}+\frac{(|n|-1)^{2}}{r^{2}}\left|v_{\theta, n}\right|^{2} .
$$

Remark 2.3 The estimate (30) implies that the Hardy inequality can be violated only in the Fourier mode $|n|=1$. 


\subsection{Biot-Savart law in $\mathcal{P}_{n} L_{\sigma}^{2}(\Omega)$}

We will analyze the operator $A_{\alpha}$ by using the streamfunction - vorticity formulation. For a given scalar field $w$ in $\Omega$ the streamfunction $\psi$ is formally defined as the solution to the Poisson equations: $-\Delta \psi=w$ in $\Omega, \psi=0$ on $\partial \Omega$. For $n \in \mathbb{Z}$ and $w \in L^{2}(\Omega)$ we set

$$
w_{n}(r)=\frac{1}{2 \pi} \int_{0}^{2 \pi} w(r \cos \theta, r \sin \theta) e^{-i n \theta} \mathrm{d} \theta .
$$

By using the Laplace operator in the polar coordinates the Poisson equations for the Fourier mode $n$ (with respect to the angular variable) is described as

$$
-\psi_{n}^{\prime \prime}-\frac{1}{r} \psi_{n}^{\prime}+\frac{n^{2}}{r^{2}} \psi_{n}=w_{n}, \quad r>1, \quad \psi_{n}(1)=0 .
$$

Here $\psi_{n}^{\prime}=\frac{\mathrm{d} \psi_{n}}{\mathrm{~d} r}$ and $\psi_{n}^{\prime \prime}=\frac{\mathrm{d}^{2} \psi_{n}}{\mathrm{~d} r^{2}}$. Let $|n| \geq 1$. Then the solution $\psi_{n}=\psi_{n}\left[w_{n}\right]$ to the ordinary differential equation (32) decaying at spatial infinity is formally given as

$$
\begin{aligned}
\psi_{n}\left[w_{n}\right](r) & =\frac{1}{2|n|}\left(-\frac{d_{n}\left[w_{n}\right]}{r^{|n|}}+\frac{1}{r^{|n|}} \int_{1}^{r} s^{1+|n|} w_{n}(s) \mathrm{d} s+r^{|n|} \int_{r}^{\infty} s^{1-|n|} w_{n}(s) \mathrm{d} s\right), \\
d_{n}\left[w_{n}\right] & =\int_{1}^{\infty} s^{1-|n|} w_{n}(s) \mathrm{d} s .
\end{aligned}
$$

The flow $V_{n}\left[w_{n}\right]$ generated by the vorticity $w_{n}$, called the Biot-Savart law, is then defined as

$$
\begin{aligned}
& V_{n}\left[w_{n}\right]=V_{r, n}\left[w_{n}\right] e^{i n \theta} \mathbf{e}_{r}+V_{\theta, n}\left[w_{n}\right] e^{i n \theta} \mathbf{e}_{\theta}, \\
& V_{r, n}\left[w_{n}\right]=\frac{i n}{r} \psi_{n}\left[w_{n}\right], \quad V_{\theta, n}\left[w_{n}\right]=-\frac{\mathrm{d}}{\mathrm{d} r} \psi_{n}\left[w_{n}\right] .
\end{aligned}
$$

Note that $V_{n}\left[w_{n}\right]$ is well defined at least when $r^{1-|n|} w_{n} \in L^{1}(1, \infty ; \mathbb{C})$, and it is straightforward to see

$$
\begin{aligned}
& \operatorname{div} V_{n}\left[w_{n}\right]=0, \quad \operatorname{rot} V_{n}\left[w_{n}\right]=w_{n} e^{i n \theta} \quad \text { in } \Omega, \\
& \mathbf{e}_{r} \cdot V_{n}\left[w_{n}\right]=0 \quad \text { on } \partial \Omega .
\end{aligned}
$$

Conversely, if the vorticity field of $v \in \mathcal{P}_{n} L_{\sigma}^{2}(\Omega),|n| \geq 1$, decays fast enough as $|x| \rightarrow \infty$ then $v$ is given by the Biot-Savart law. To prove this we first observe

Lemma 2.4 Let $v \in \mathcal{P}_{n} W^{1,2}(\Omega)^{2}$ for some $|n| \geq 1$. Assume in addition that $r^{1-|n|} e^{-i n \theta} \operatorname{rot} v$ belongs to $L^{1}(1, \infty ; \mathbb{C})$. Then $V_{n}\left[w_{n}\right]$ with $w_{n}=e^{-i n \theta} \operatorname{rot} v$ satisfies

$$
\lim _{r \rightarrow \infty}\left(\left|V_{r, n}\left[w_{n}\right](r)\right|+\left|V_{\theta, n}\left[w_{n}\right](r)\right|\right)=0 .
$$

Remark 2.5 If $|n| \geq 2$ then the condition $r^{1-|n|} e^{-i n \theta} \operatorname{rot} v \in L^{1}(1, \infty ; \mathbb{C})$ is automatically satisfied for $v \in \mathcal{P}_{n} W^{1,2}(\Omega)^{2}$.

Proof of Lemma 2.4. Let $v=v_{n}=v_{r, n} e^{i n \theta} \mathbf{e}_{r}+v_{\theta, n} e^{i n \theta} \mathbf{e}_{\theta}$ in the polar coordinates. By Lemma 2.1 we see $w_{n}=\frac{1}{r} \partial_{r}\left(r v_{\theta, n}\right)-\frac{i n}{r} v_{r, n}$, and thus the integration by parts yields

$$
\begin{aligned}
I(r) & :=\frac{1}{r^{1+|n|}} \int_{1}^{r} s^{1+|n|} w_{n}(s) \mathrm{d} s \\
& =v_{\theta, n}(r)-\frac{v_{\theta, n}(1)}{r^{1+|n|}}-\frac{|n|}{r^{1+|n|}} \int_{1}^{r} s^{|n|} v_{\theta, n}(s) \mathrm{d} s-\frac{i n}{r^{1+|n|}} \int_{1}^{r} s^{|n|} v_{r, n}(s) \mathrm{d} s,
\end{aligned}
$$


and

$$
\begin{aligned}
I I(r) & :=r^{|n|-1} \int_{r}^{\infty} s^{1-|n|} w_{n}(s) \mathrm{d} s \\
& =-v_{\theta, n}(r)+|n| r^{|n|-1} \int_{r}^{\infty} s^{-|n|} v_{\theta, n}(s) \mathrm{d} s-i n r^{|n|-1} \int_{r}^{\infty} s^{-|n|} v_{r, n}(s) \mathrm{d} s .
\end{aligned}
$$

We note that $v_{n} \in \mathcal{P}_{n} L^{2}(\Omega)^{2}$ is equivalent with $\int_{1}^{\infty}\left(\left|v_{r, n}\right|^{2}+\left|v_{\theta, n}\right|^{2}\right) r \mathrm{~d} r<\infty$. Hence it is easy to see that if $v_{n} \in \mathcal{P}_{n} W^{1,2}(\Omega)^{2}$ then $|I(r)|$ and $|I I(r)|$ converge to 0 as $r \rightarrow \infty$, which implies (36) by the definition of $V_{n}$ in (34). The proof is complete.

The next proposition verifies the Biot-Savart low for $v \in W^{1,2}(\Omega)^{2} \cap \mathcal{P}_{n} L_{\sigma}^{2}(\Omega)$ in the case $|n| \geq 1$.

Proposition 2.6 Let $v \in W^{1,2}(\Omega)^{2} \cap \mathcal{P}_{n} L_{\sigma}^{2}(\Omega)$ for some $|n| \geq 1$. Assume in addition that $r^{1-|n|} e^{-i n \theta} \operatorname{rot} v$ belongs to $L^{1}(1, \infty ; \mathbb{C})$. Then $v=V_{n}\left[w_{n}\right]$ with $w_{n}=e^{-i n \theta} \operatorname{rot} v$.

Proof. Let $v=v_{n}=v_{r, n} e^{i n \theta} \mathbf{e}_{r}+v_{\theta, n} e^{i n \theta} \mathbf{e}_{\theta}$. Then $w_{n}=e^{-i n \theta} \operatorname{rot} v$ depends only on $r$ and $V_{n}\left[w_{n}\right]$ satisfies (35). Then $u_{n}=v_{n}-V_{n}\left[w_{n}\right]$ satisfies $\operatorname{div} u_{n}=0$ and $\operatorname{rot} u_{n}=0$ in $\Omega$, and $\mathbf{e}_{r} \cdot u_{n}=0$ on $\partial \Omega$. Hence $u_{n}$ is harmonic and smooth in $\Omega$. By writing $u_{n}=$ $u_{r, n} e^{i n \theta} \mathbf{e}_{r}+u_{\theta, n} e^{i n \theta} \mathbf{e}_{\theta}$ and using Lemma 2.1, we see that $u_{r, n}$ satisfies

$$
-u_{r, n}^{\prime \prime}-\frac{3}{r} u_{r, n}^{\prime}+\frac{n^{2}-1}{r^{2}} u_{r, n}=0, \quad r>1,\left.\quad u_{r, n}\right|_{r=1}=0 .
$$

Let $\chi_{R} \in C_{0}^{\infty}([1, \infty)), R \gg 1$, be a cut-off function such that $\chi_{R}=1$ on $[1, R], \chi_{R}=0$ on $[2 R, \infty)$, and $\left\|\left(\chi_{R}\right)^{\prime}\right\|_{L^{\infty}} \leq C / R$. Multiplying both sides with $\chi_{R} \bar{u}_{r, n} r$ and integrating over $(1, \infty)$, we have

$$
\begin{aligned}
& \int_{1}^{\infty}\left|u_{r, n}^{\prime}\right|^{2} \chi_{R} r \mathrm{~d} r+\left(n^{2}-1\right) \int_{1}^{\infty} \frac{\left|u_{r, n}\right|^{2}}{r^{2}} r \mathrm{~d} r \\
& =-\Re \int_{1}^{\infty} u_{r, n}^{\prime} \bar{u}_{r, n} \chi_{R}^{\prime} r \mathrm{~d} r+2 \Re \int_{1}^{\infty} u_{r, n}^{\prime} \bar{u}_{r, n} \chi_{R} \mathrm{~d} r \\
& =\Re \int_{1}^{\infty}\left|u_{r, n}\right|^{2}\left(\frac{1}{2}\left(\chi_{R}^{\prime} r\right)^{\prime}-\chi_{R}^{\prime}\right) \mathrm{d} r .
\end{aligned}
$$

Lemma 2.4 and the assumption $v \in \mathcal{P}_{n} L^{2}(\Omega)$ implies that the right-hand side of (37) converges to 0 as $R \rightarrow \infty$. Hence we conclude that $u_{r, n}=0$, which also implies $u_{\theta, n}=0$ since $0=\operatorname{div} u_{n}=\frac{1}{r}\left(r u_{r, n}\right)^{\prime}+\frac{i n}{r} u_{\theta, n}$ by Lemma 2.1 and $|n| \geq 1$. The proof is complete.

Corollary 2.7 Let $f \in \mathcal{P}_{n} L^{2}(\Omega)^{2}$ for some $|n| \geq 1$. Assume that $\operatorname{rot} f=0$ in $\Omega$ in the sense of distributions. Then $f=\nabla p$ for some $p \in \hat{W}^{1,2}(\Omega)=\left\{q \in L_{\text {loc }}^{2}(\Omega) \mid \nabla q \in\right.$ $\left.L^{2}(\Omega)^{2}\right\}$.

Proof. It suffices to show $\langle f, v\rangle_{L^{2}(\Omega)}=0$ for any $v \in C_{0, \sigma}^{\infty}(\Omega)$. Since $f=\mathcal{P}_{n} f$ by the assumption and since $\mathcal{P}_{n}$ is an orthogonal projection in $L^{2}(\Omega)^{2}$ which satisfies $\mathcal{P}_{n} C_{0, \sigma}^{\infty}(\Omega) \subset C_{0, \sigma}^{\infty}(\Omega)$, we may assume that $v \in \mathcal{P}_{n} C_{0, \sigma}^{\infty}(\Omega)$. By Proposition 2.6 we have $v=V_{n}\left[w_{n}\right]$ for some $w_{n} \in C_{0}^{\infty}(1, \infty)$. Since $v$ belongs to $C_{0, \sigma}^{\infty}(\Omega)$ the streamfunction $\psi$ must satisfy $\psi_{n}(1)=\psi_{n}^{\prime}(1)=0$. Then, the fact $w_{n}$ is identically zero near $r=1$ 
implies that $\psi_{n}$ also vanishes near $r=1$ due to the equation (32). With the notion of $f=f_{n}=f_{r, n} e^{i n \theta} \mathbf{e}_{r}+f_{\theta, n} e^{i n \theta} \mathbf{e}_{\theta}$, we see

$$
\begin{aligned}
\langle f, v\rangle_{L^{2}(\Omega)} & =\int_{1}^{\infty} f_{r, n}(r) \overline{V_{r, n}\left[w_{n}\right](r)} r \mathrm{~d} r+\int_{1}^{\infty} f_{\theta, n}(r) \overline{V_{\theta, n}\left[w_{n}\right](r)} r \mathrm{~d} r \\
& =\int_{1}^{\infty}\left(f_{r, n}(r) \overline{\frac{i n}{r} \psi_{n}(r)}-f_{\theta, n}(r) \overline{\psi_{n}^{\prime}(r)}\right) r \mathrm{~d} r .
\end{aligned}
$$

Let $\chi_{R} \in C_{0}^{\infty}([1, \infty))$ be the cut-off function as in the proof of Proposition 2.6. We recall that, by Lemma 2.1, $\operatorname{rot} f=0$ in $\Omega$ in the sense of distributions is equivalent with $\frac{1}{r}\left(r f_{\theta, n}\right)^{\prime}-\frac{i n}{r} f_{r, n}=0$ in $(1, \infty)$ in the sense of distributions. Then, since $\chi_{R} \psi_{n} \in$ $C_{0}^{\infty}((1, \infty))$ as is already observed, it follows that

$$
\int_{1}^{\infty}\left(f_{r, n}(r) \overline{\frac{i n}{r} \chi_{R} \psi_{n}(r)}-f_{\theta, n}(r) \overline{\left(\chi_{R} \psi_{n}\right)^{\prime}(r)}\right) r \mathrm{~d} r=0 .
$$

Therefore,

$$
\begin{aligned}
\langle f, v\rangle_{L^{2}(\Omega)}= & \int_{1}^{\infty}\left(f_{r, n}(r) \overline{\frac{i n}{r}\left(1-\chi_{R}\right) \psi_{n}(r)}-f_{\theta, n}(r) \overline{\left(\left(1-\chi_{R}\right) \psi_{n}\right)^{\prime}(r)}\right) r \mathrm{~d} r \\
= & \int_{1}^{\infty}\left(1-\chi_{R}\right)\left(f_{r, n}(r) \overline{\frac{i n}{r} \psi_{n}(r)}-f_{\theta, n}(r) \overline{\left(\psi_{n}\right)^{\prime}(r)}\right) r \mathrm{~d} r \\
& +\int_{1}^{\infty} f_{\theta, n}(r)\left(\chi_{R}\right)^{\prime} \overline{\psi_{n}(r)} r \mathrm{~d} r \\
= & \left\langle f,\left(1-\chi_{R}\right) v\right\rangle_{L^{2}(\Omega)}+\int_{1}^{\infty} f_{\theta, n}(r)\left(\chi_{R}\right)^{\prime} \overline{\psi_{n}(r)} r \mathrm{~d} r .
\end{aligned}
$$

It is straightforward to see that the first term of (39) converges to 0 as $R \rightarrow \infty$. As for the second term of (39), we have from $\left|\psi_{n}(r)\right| \leq C r^{-|n|}$ for $r \gg 1$,

$$
\left|\int_{1}^{\infty} f_{\theta, n}(r)\left(\chi_{R}\right)^{\prime} \overline{\psi_{n}(r)} r \mathrm{~d} r\right| \leq C R^{-1}\|f\|_{L^{2}(\Omega)} \rightarrow 0 \quad(R \rightarrow \infty) .
$$

Hence, $\langle f, v\rangle_{L^{2}(\Omega)}=0$ for any $v \in C_{0, \sigma}^{\infty}(\Omega)$, which implies $f=\nabla p$ for some $p \in$ $\hat{W}^{1,2}(\Omega)$. The proof is complete.

\subsection{Decomposition of the perturbed Stokes operator $A_{\alpha}$}

In this section we study the perturbation term $\mathbb{P}(U \cdot \nabla v+v \cdot \nabla U)$. Let us start from the following

Lemma 2.8 Let $U(x)=\frac{x^{\perp}}{|x|^{2}}$ and $v \in W^{1,2}(\Omega)^{2} \cap L_{\sigma}^{2}(\Omega)$. Then

$$
\mathbb{P}(U \cdot \nabla v+v \cdot \nabla U)=\mathbb{P}\left(U^{\perp} \operatorname{rot} v\right) \quad \text { in } \Omega .
$$

Proof. We recall that any $u \in W^{1,2}(\Omega)^{2} \cap L_{\sigma}^{2}(\Omega)$ satisfies the identity

$$
u \cdot \nabla u=\frac{1}{2} \nabla|u|^{2}+u^{\perp} \operatorname{rot} u, \quad u^{\perp}=\left(-u_{2}, u_{1}\right) .
$$


We also note that the Helmholtz projection $\mathbb{P}$ is bounded from $L^{p}(\Omega)^{2}$ to $L_{\sigma}^{p}(\Omega)$ for $1<$ $p<\infty$; cf. Simader and Sohr [43]. Hence, we have for $u=U+v$,

$$
\begin{aligned}
\mathbb{P}(u \cdot \nabla u)=\mathbb{P}\left(u^{\perp} \operatorname{rot} u\right) & =\mathbb{P}\left(U^{\perp} \operatorname{rot} U+v^{\perp} \operatorname{rot} v+U^{\perp} \operatorname{rot} v+v^{\perp} \operatorname{rot} U\right) \\
& =\mathbb{P}\left(v^{\perp} \operatorname{rot} v+U^{\perp} \operatorname{rot} v\right) .
\end{aligned}
$$

Here we have used $\operatorname{rot} U=0$ for $x \neq 0$. On the other hand, we have

$$
\mathbb{P}(v \cdot \nabla v)=\mathbb{P}\left(v^{\perp} \operatorname{rot} v\right), \quad \mathbb{P}(U \cdot \nabla U)=0 .
$$

Comparing these identities, we obtain (40). The proof is complete.

By Lemma 2.8 the perturbed Stokes operator $A_{\alpha}$ is written as

$$
A_{\alpha} v=A v+\alpha \mathbb{P}\left(U^{\perp} \operatorname{rot} v\right), \quad U^{\perp}(x)=-\frac{x}{|x|^{2}} .
$$

The expression (41) makes it easier to see that $\mathcal{P}_{l} L_{\sigma}^{2}(\Omega)$ is invariant under the action of $A_{\alpha}$. Indeed, we have

Lemma 2.9 Let $n \in \mathbb{Z}$ and let $v \in W^{1,2}(\Omega)^{2}$. Then it follows that

$$
\mathcal{P}_{n}\left(\frac{x}{|x|^{2}} \operatorname{rot} v\right)=\frac{x}{|x|^{2}} \operatorname{rot} \mathcal{P}_{n} v .
$$

In particular, we have

$$
\mathcal{P}_{0}\left(\frac{x}{|x|^{2}} \operatorname{rot} v\right)=\nabla p_{0}
$$

where $p_{0}=p_{0}(|x|)$ with

$$
p_{0}(r)=-\int_{r}^{\infty} \frac{\operatorname{rot} \mathcal{P}_{0} v}{s} \mathrm{~d} s .
$$

Proof. Set $w=\operatorname{rot} v$. We note that $\frac{x}{|x|^{2}} w=u_{r} \mathbf{e}_{r}$ with $u_{r}=r^{-1} w$. Thus, the definition of $\mathcal{P}_{n}$ implies $\mathcal{P}_{n}\left(\frac{x}{|x|^{2}} w\right)=r^{-1} w_{n} e^{i n \theta} \mathbf{e}_{r}$, where $w_{n}$ is as in (31). Since $\operatorname{rot} \mathcal{P}_{n} v=w_{n} e^{i n \theta}$, the identity (42) holds. When $n=0$ we have $\operatorname{rot} \mathcal{P}_{0} v=w_{0}$ with $w_{0}=w_{0}(r)$. Hence (43) follows. The proof is complete.

Proposition 2.10 For each $m \in \mathbb{N} \cup\{0\}$ the perturbed Stokes operator $A_{\alpha}$ in $L_{\sigma}^{2}(\Omega)$ is diagonalized as

$$
A_{\alpha}=\left.\left.A\right|_{L_{\sigma, \text { rad }}^{2}} \oplus A_{\alpha}\right|_{\mathcal{Q}_{m} L_{\sigma}^{2}} \oplus\left(\left.\oplus_{1 \leq|n| \leq m} A_{\alpha}\right|_{\mathcal{P}_{n} L_{\sigma}^{2}}\right) .
$$

Proof. From (23), (27), and Lemma 2.9, the following invariance holds.

$$
\mathcal{P}_{n} A_{\alpha} \subset A_{\alpha} \mathcal{P}_{n}, \quad \mathcal{Q}_{m} A_{\alpha} \subset A_{\alpha} \mathcal{Q}_{m} .
$$

Moreover, (43) implies $\mathbb{P}\left(U^{\perp} \operatorname{rot} v\right)=0$ for $v \in D_{L^{2}}\left(A_{\alpha}\right) \cap L_{\sigma, r a d}^{2}(\Omega)$. Hence we have $\left.A_{\alpha}\right|_{L_{\sigma, \text { rad }}^{2}}=\left.A\right|_{L_{\sigma, \text { rad }}^{2}}$. The proof is complete. 


\subsection{Relatively compactness of $A_{\alpha}-A$}

Recalling the representation (41), we show that $\alpha \mathbb{P}\left(U^{\perp}\right.$ rot $)$ is relatively compact with respect to the Stokes operator $A$.

Lemma 2.11 Set $\rho(x)=\left(1+|x|^{2}\right)^{1 / 2}$. Then it follows that for any $v \in W^{2,2}(\Omega)^{2}$,

$$
\left\|\rho^{\frac{1}{2}} \mathbb{P}\left(U^{\perp} \operatorname{rot} v\right)\right\|_{L^{2}(\Omega)}+\left\|\nabla \mathbb{P}\left(U^{\perp} \operatorname{rot} v\right)\right\|_{L^{2}(\Omega)} \leq C\|\operatorname{rot} v\|_{W^{1,2}(\Omega)} .
$$

Proof. Firstly we recall that the Helmholtz projection is bounded also in the weighted $L^{p}$ spaces for $1<p<\infty$ if the weight belongs to the Muckenhoupt class $\mathcal{A}_{p}$; see Farwig and Sohr [11, Theorem 1.3]. In particular, the result of [11, Theorem 1.3] leads to the inequality $\left\|\rho^{1 / 2} \mathbb{P} f\right\|_{L^{2}(\Omega)} \leq C\left\|\rho^{1 / 2} f\right\|_{L^{2}(\Omega)}$, which yields

$$
\left\|\rho^{\frac{1}{2}} \mathbb{P}\left(U^{\perp} \operatorname{rot} v\right)\right\|_{L^{2}(\Omega)} \leq C\|\operatorname{rot} v\|_{L^{2}(\Omega)}
$$

by the pointwise estimate $|U(x)| \leq|x|^{-1}$. As for the derivative estimate, we observe that

$$
\left\|\nabla \mathbb{P}\left(U^{\perp} \operatorname{rot} v\right)\right\|_{L^{2}(\Omega)} \leq\left\|\nabla\left(U^{\perp} \operatorname{rot} v\right)\right\|_{L^{2}(\Omega)}+\left\|\nabla^{2} p\right\|_{L^{2}(\Omega)},
$$

where $p \in L_{l o c}^{2}(\Omega)$ with $\nabla p \in L^{2}(\Omega)$ is the solution to the Neumann problem

$$
\Delta p=\operatorname{div}\left(U^{\perp} \operatorname{rot} v\right) \text { in } \Omega, \quad \partial_{n} p=-\operatorname{rot} v \text { on } \partial \Omega .
$$

Thus the elliptic estimate for the Neumann problem yields $\left\|\nabla^{2} p\right\|_{L^{2}(\Omega)} \leq C\|\operatorname{rot} v\|_{W^{1,2}(\Omega)}$, which gives (45). The proof is complete.

By the Rellich theorem Lemma 2.11 implies that $A_{\alpha}$ is a relatively compact perturbation of the Stokes operator $A$. To apply the perturbation theory for sectorial operators let us recall some terminologies in Kato [27]. A closed operator $T: D_{X}(T) \subset X \rightarrow X$ in a Banach space $X$ is called semi-Fredholm if $R(T)$, the range of $T$, is closed and either the dimension of the null space $\left\{u \in D_{X}(T) \mid T u=0\right\}$ or the codimension of $R(T)$ is finite. Then the semiFredholm domain $O_{S F}(T)$ and the essential spectrum $\sigma_{e s s}(T)$ of $T$ are respectively defined as

$$
O_{S F}(T)=\{\zeta \in \mathbb{C} \mid T-\zeta \text { is semiFredholm }\}, \quad \sigma_{\text {ess }}(T)=\mathbb{C} \backslash O_{S F}(T) .
$$

It is well known that $O_{S F}(T)$ is open and thus $\sigma_{e s s}(T)$ is closed; see [27, IV-6, pp.242] for details. Moreover, if $\lambda \in O_{S F}(T)$ is an isolated point of $\sigma(T)$ then $\lambda$ is an eigenvalue (since the index itself must be zero in this case) and the rank of the spectral projection associated with $\lambda$ must be finite; this is a consequence of [27, IV-5, Theorems 5.10, 5.28]. In other words, in that case $\lambda$ is an isolated eigenvalue of $T$ with finite algebraic multiplicity.

Proposition 2.12 The spectrum $\sigma\left(-A_{\alpha}\right)$ is equal to $\overline{\mathbb{R}_{-}} \cup \sigma_{\text {disc }}\left(-A_{\alpha}\right)$, and

$$
\sigma_{\text {disc }}\left(-A_{\alpha}\right)=\bigcup_{n \in \mathbb{Z} \backslash\{0\}} \sigma_{\text {disc }}\left(-\left.A_{\alpha}\right|_{\mathcal{P}_{n} L_{\sigma}^{2}}\right) \backslash \overline{\mathbb{R}_{-}} .
$$

Remark 2.13 In fact, by arguing as in [10, Lemma 3.3] we can show

$$
\sigma_{e s s}\left(-\left.A_{\alpha}\right|_{\mathcal{P}_{n} L_{\sigma}^{2}}\right)=\sigma_{e s s}\left(-\left.A\right|_{\mathcal{P}_{n} L_{\sigma}^{2}}\right)=\overline{\mathbb{R}_{-}},
$$

and hence, (46) is simply replaced by $\sigma_{d i s c}\left(-A_{\alpha}\right)=\bigcup_{n \in \mathbb{Z} \backslash\{0\}} \sigma_{d i s c}\left(-\left.A_{\alpha}\right|_{\mathcal{P}_{n} L_{\sigma}^{2}}\right)$. But we omit the proof of (47) in this paper, for (46) is enough for our purpose to establish Theorem 1.5 . 
Proof of Proposition 2.12. It is well known that the Stokes operator, which is self-adjoint, satisfies $\sigma(-A)=\overline{\mathbb{R}_{-}}$; cf. Ladyzhenskaya [34]. Furthermore, as in [10, Lemma 2.6] one can also show that $\sigma_{e s s}(-A)=\sigma(-A)=\overline{\mathbb{R}_{-}}$; for completeness we give a proof slightly different from [10, Lemma 2.6]. Note that we have already known that $\sigma_{\text {ess }}(-A) \subset$ $\sigma(-A)=\overline{\mathbb{R}_{-}}$. If $\lambda \in \overline{\mathbb{R}_{-}}(=\sigma(-A))$ belongs to $O_{S F}(T)$ then there is an open ball $B$ such that $\lambda \in B \subset O_{S F}(T)$, for $O_{S F}(T)$ is an open set. Then the index of $-A-\zeta$ is constant in this connected open set $B$ by the stability theorem [27, IV-5, Theorem 5.26], and moreover, the dimension of the null space of $-A-\zeta$ is also constant in $B$ except for an isolated set of values of $\zeta$ in $B$; cf. [27, Theorem 3.1]. Since $B$ includes a point of the resolvent set, the above statements imply that the index of $-A-\zeta$ is zero in $B$, and the dimension of the null space is also zero in $B$ except for some isolated points. Hence there is $\zeta \in B \cap \overline{\mathbb{R}_{-}}$, which is included in $O_{S F}(T)$, such that $\zeta \in \rho(-A)$. This contradicts with $\sigma(-A)=\overline{\mathbb{R}_{-}}$, and we conclude that $\sigma_{\text {ess }}(-A)=\sigma(-A)=\overline{\mathbb{R}_{-}}$. Since $A_{\alpha}$ is $A$-compact by Lemma 2.11, we have from [27, IV-5, Theorem 5.35] that $\sigma_{\text {ess }}\left(-A_{\alpha}\right)=\sigma_{\text {ess }}(-A)=\overline{\mathbb{R}_{-}}$, which yields $O_{S F}\left(-A_{\alpha}\right)=\mathbb{C} \backslash \overline{\mathbb{R}_{-}}$for any $\alpha \in \mathbb{R}$. Note that $\mathbb{C} \backslash \overline{\mathbb{R}_{-}}$is connected and includes the resolvent set, and thus, the index of $-A_{\alpha}-\zeta$ must be zero for any $\zeta \in \mathbb{C} \backslash \overline{\mathbb{R}_{-}}$. Moreover, the dimension of the null space of $-A_{\alpha}-\zeta$ is also zero in $\mathbb{C} \backslash \overline{\mathbb{R}_{-}}$except for some isolated points (see also [27, IV-6, pp. 243]). Hence one can conclude that $O_{S F}\left(-A_{\alpha}\right)=\mathbb{C} \backslash \overline{\mathbb{R}_{-}}$consists of the resolvent set and the isolated points of $\sigma\left(-A_{\alpha}\right)$ which are eigenvalues of $-A_{\alpha}$ with finite algebraic multiplicities. Thus we have proved $\sigma\left(-A_{\alpha}\right)=\overline{\mathbb{R}}_{-} \cup \sigma_{\text {disc }}\left(-A_{\alpha}\right)$ and $\sigma_{\text {disc }}\left(-A_{\alpha}\right) \subset \mathbb{C} \backslash \overline{\mathbb{R}_{-}}$. The identity (46) follows from $\sigma\left(-A_{\alpha}\right)=\overline{\mathbb{R}_{-}} \cup \sigma_{\text {disc }}\left(-A_{\alpha}\right)$, $\sigma_{\text {disc }}\left(-A_{\alpha}\right) \subset \mathbb{C} \backslash \overline{\mathbb{R}_{-}}$, Proposition 2.10, and $\sigma_{\text {disc }}\left(-\left.A\right|_{L_{\sigma, \text { rad }}^{2}}\right)=\emptyset$. The proof is complete.

\section{Spectral analysis of $A_{\alpha}$}

In this section we study the discrete spectrum $\sigma_{\text {disc }}\left(-A_{\alpha}\right)$ in the general case $\alpha \in \mathbb{R}$. To this end let us consider the equations

$$
\lambda v+A_{\alpha} v=f, \quad \lambda \in \mathbb{C} \backslash \overline{\mathbb{R}_{-}}, v \in D_{L^{2}}\left(A_{\alpha}\right), \quad f \in L_{\sigma}^{2}(\Omega) .
$$

The key idea is to analyze (48) in each invariant subspace $\mathcal{P}_{n} L_{\sigma}^{2}(\Omega)$ by using the associated streamfunction - vorticity formulation, which enables us to obtain a representation formula for solutions to (48). To this end we firstly assume that $f \in C_{0, \sigma}^{\infty}(\Omega)$. By the elliptic regularity of the Stokes operator it is easy to see that any solution $v$ to (48) satisfies $v \in$ $W^{k, 2}(\Omega)^{2}$ for any $k \in \mathbb{N}$ in this case. Let us consider the equations for the vorticity field $w=\operatorname{rot} v$ :

$$
\lambda w-\Delta w+\alpha U \cdot \nabla w=\operatorname{rot} f .
$$

This equation is easily derived by taking rot in (48) and from (41). From $U(x)=\frac{x^{\perp}}{|x|^{2}}$ the equation (49) in the polar coordinates is described as

$$
\lambda w-\left(\partial_{r}^{2}+\frac{1}{r} \partial_{r}+\frac{1}{r^{2}} \partial_{\theta}^{2}\right) w+\frac{\alpha}{r^{2}} \partial_{\theta} w=h, \quad h=\operatorname{rot} f .
$$

Let us assume in addition that $f$ and $v$ belong to $\mathcal{P}_{n} L_{\sigma}^{2}(\Omega)$ for some $n \in \mathbb{Z} \backslash\{0\}$, and set

$$
\begin{aligned}
& f=f_{n}=f_{r, n} e^{i n \theta} \mathbf{e}_{r}+f_{\theta, n} e^{i n \theta} \mathbf{e}_{\theta}, \\
& v=v_{n}=v_{r, n} e^{i n \theta} \mathbf{e}_{r}+v_{\theta, n} e^{i n \theta} \mathbf{e}_{\theta} .
\end{aligned}
$$


We have $\operatorname{rot} f=h=h_{n} e^{i n \theta}, \operatorname{rot} v=w=w_{n} e^{i n \theta}$, where $h_{n}$ and $w_{n}$ are functions on the $r$ variable defined as in (31). Then (50) is reduced to

$$
-w_{n}^{\prime \prime}-\frac{1}{r} w_{n}^{\prime}+\left(\lambda+\frac{n^{2}+i \alpha n}{r^{2}}\right) w_{n}=h_{n}, \quad h_{n}=\frac{1}{r}\left(r f_{\theta, n}\right)^{\prime}-\frac{i n}{r} f_{r, n} .
$$

Let $I_{\mu}(z)$ be the modified Bessel function of first kind of order $\mu$ :

$$
I_{\mu}(z)=\left(\frac{z}{2}\right)^{\mu} \sum_{m=0}^{\infty} \frac{1}{m ! \Gamma(\mu+m+1)}\left(\frac{z}{2}\right)^{2 m}, \quad z \in \mathbb{C} \backslash \overline{\mathbb{R}_{-}} .
$$

Here $z^{\mu}=e^{\mu \log z}$ and $\log z=\log |z|+i \arg z$ is the principal branch of the logarithm of $z=|z| e^{i \theta},-\pi<\arg z=\theta<\pi$, while $\Gamma(z)$ is the Gamma function. For $\mu \notin \mathbb{Z}$ the Bessel function $K_{\mu}$ defined as (10) is related to $I_{\mu}$ by the formula

$$
K_{\mu}(z)=\frac{\pi}{2} \cdot \frac{I_{-\mu}(z)-I_{\mu}(z)}{\sin (\mu \pi)}, \quad \Re(z)>0 .
$$

Note that the right-hand side of (53) gives an analytic extension of $K_{\mu}(z)$ to $\mathbb{C} \backslash \overline{\mathbb{R}_{-}}$. It is well known that $K_{\mu}$ and $I_{\mu}$ are independent solutions to the ordinary differential equation

$$
-\frac{\mathrm{d}^{2} \omega}{\mathrm{d} z^{2}}-\frac{1}{z} \frac{\mathrm{d} \omega}{\mathrm{d} z}+\left(1+\frac{\mu^{2}}{z^{2}}\right) \omega=0
$$

with the Wronskian

$$
W_{\mu}(z)=\operatorname{det}\left(\begin{array}{ll}
K_{\mu}(z) & I_{\mu}(z) \\
\frac{\mathrm{d} K_{\mu}}{\mathrm{d} z}(z) & \frac{\mathrm{d} I_{\mu}}{\mathrm{d} z}(z)
\end{array}\right)=\frac{1}{z} .
$$

Set $\mu_{n}(\alpha)=\left(n^{2}+i \alpha n\right)^{1 / 2}, \Re\left(\mu_{n}(\alpha)\right)>0$. Then $K_{\mu_{n}(\alpha)}(\sqrt{\lambda} r)$ and $I_{\mu_{n}(\alpha)}(\sqrt{\lambda} r)$ with $\Re(\sqrt{\lambda})>0$ are independent solutions to the homogeneous equation of (51) and their Wronskian is $r^{-1}$. As is well known, the function $K_{\mu}(z)$ decays exponentially as $|z| \rightarrow \infty$, $\Re(z)>0$, while $I_{\mu}(z)$ grows exponentially; see Lemma A.3. Hence the solution to (51) which decays for $r \rightarrow \infty$ is represented as

$$
w_{n}(r)=c_{n} K_{\mu_{n}(\alpha)}(\sqrt{\lambda} r)+\Phi_{n, \lambda}\left[f_{n}\right](r)
$$

where $c_{n}$ is a constant and

$$
\begin{aligned}
\Phi_{n, \lambda}\left[f_{n}\right](r)= & K_{\mu_{n}(\alpha)}(\sqrt{\lambda} r) \int_{1}^{r} s I_{\mu_{n}(\alpha)}(\sqrt{\lambda} s) h_{n}(s) \mathrm{d} s \\
& +I_{\mu_{n}(\alpha)}(\sqrt{\lambda} r) \int_{r}^{\infty} s K_{\mu_{n}(\alpha)}(\sqrt{\lambda} s) h_{n}(s) \mathrm{d} s \\
h_{n}= & \frac{1}{r}\left(r f_{\theta, n}\right)^{\prime}-\frac{i n}{r} f_{r, n} .
\end{aligned}
$$

For simplicity of notations we will sometime write $\mu_{n}$ for $\mu_{n}(\alpha)$. By performing the inte- 
gration by parts in (57) and by using (324) and (327), we have for $f_{n} \in \mathcal{P}_{n} C_{0, \sigma}^{\infty}(\Omega)$,

$$
\begin{aligned}
& \Phi_{n, \lambda}\left[f_{n}\right](r) \\
= & K_{\mu_{n}}(\sqrt{\lambda} r)\left(-\sqrt{\lambda} \int_{1}^{r} \frac{\mathrm{d} I_{\mu_{n}}}{\mathrm{~d} z}(\sqrt{\lambda} s) f_{\theta, n}(s) s \mathrm{~d} s-i n \int_{1}^{r} I_{\mu_{n}}(\sqrt{\lambda} s) f_{r, n}(s) \mathrm{d} s\right) \\
+ & I_{\mu_{n}}(\sqrt{\lambda} r)\left(-\sqrt{\lambda} \int_{r}^{\infty} \frac{\mathrm{d} K_{\mu_{n}}}{\mathrm{~d} z}(\sqrt{\lambda} s) f_{\theta, n}(s) s \mathrm{~d} s-i n \int_{r}^{\infty} K_{\mu_{n}}(\sqrt{\lambda} s) f_{r, n}(s) \mathrm{d} s\right) \\
= & -K_{\mu_{n}}(\sqrt{\lambda} r)\left(\int_{1}^{r} I_{\mu_{n}}(\sqrt{\lambda} s)\left(\mu f_{\theta, n}(s)+i n f_{r, n}(s)\right) \mathrm{d} s\right. \\
& \left.+\sqrt{\lambda} \int_{1}^{r} I_{\mu_{n}+1}(\sqrt{\lambda} s) f_{\theta, n}(s) s \mathrm{~d} s\right) \\
+ & I_{\mu_{n}}(\sqrt{\lambda} r)\left(\int_{r}^{\infty} K_{\mu_{n}}(\sqrt{\lambda} s)\left(\mu f_{\theta, n}(s)-i n f_{r, n}(s)\right) \mathrm{d} s\right. \\
& \left.+\sqrt{\lambda} \int_{r}^{\infty} K_{\mu_{n}-1}(\sqrt{\lambda} s) f_{\theta, n}(s) s \mathrm{~d} s\right)
\end{aligned}
$$

We note that the representation (58) makes sense for any $f_{n} \in \mathcal{P}_{n} L^{2}(\Omega)^{2}$ and is equivalent with (57) if $f_{n} \in \mathcal{P}_{n} W_{0}^{1,2}(\Omega)^{2}$. If $f_{n} \in C_{0, \sigma}^{\infty}(\Omega)$ in addition then it is not difficult to see from the estimates of $K_{\mu}(z)$ (cf. Lemma A.3) that the vorticity $w_{n}$, which is now represented as (56), decays exponentially as $r \rightarrow \infty$. Hence, Proposition 2.6 implies that any solution $v \in D_{L_{\sigma}^{2}}\left(A_{\alpha}\right) \cap \mathcal{P}_{n} L_{\sigma}^{2}(\Omega)$ to (48) for $f=f_{n} \in \mathcal{P}_{n} C_{0, \sigma}^{\infty}(\Omega)$, if it exists, must be given by the Biot-Savart law (34), i.e., $v=V_{n}\left[w_{n}\right]$ with $w_{n}$ expressed as in (56) for some constant $c_{n}$. The determination of the constant $c_{n}$ is the key in this streamfunction-vorticity formulation, and it will be revealed in the next section that the possibility to determine unique $c_{n}$ is directly connected with the problem whether $\lambda$ is an eigenvalue or a resolvent of $-A_{\alpha}$.

\subsection{Characterization of discrete spectrum}

Let us recall that $\sigma_{d i s c}\left(-A_{\alpha}\right)$ is the set of the discrete spectrum of $-A_{\alpha}$, i.e, the set of the isolated eigenvalues of $-A_{\alpha}$ with finite algebraic multiplicity. By Proposition 2.12 we have already known that $\sigma\left(-A_{\alpha}\right)=\overline{\mathbb{R}_{-}} \cup \sigma_{\text {disc }}\left(-A_{\alpha}\right)$. Set

$$
c_{n, \lambda}\left[f_{n}\right]=d_{n}\left[\Phi_{n, \lambda}\left[f_{n}\right]\right]=\int_{1}^{\infty} s^{1-|n|} \Phi_{n, \lambda}\left[f_{n}\right](s) \mathrm{d} s,
$$

where $\Phi_{n, \lambda}\left[f_{n}\right]$ is defined as (58). The following lemma is a key to understand the structure of $\sigma_{\text {disc }}\left(-A_{\alpha}\right)$.

Lemma 3.1 Let $f=f_{n} \in \mathcal{P}_{n} C_{0, \sigma}^{\infty}(\Omega)$ for some $n \in \mathbb{Z} \backslash\{0\}$ and let $w_{n}$ be the function given as (56) for some constant $c_{n}$. Then the velocity $V_{n}\left[w_{n}\right]$ defined by the Biot-Savart law (34) is a solution to (48) if and only if $d_{n}\left[w_{n}\right]=0$, that is,

$$
c_{n} F_{n}(\sqrt{\lambda} ; \alpha)+c_{n, \lambda}\left[f_{n}\right]=0 .
$$

Here $d_{n}\left[w_{n}\right], F_{n}(\sqrt{\lambda} ; \alpha)$, and $c_{n, \lambda}[\cdot]$ are defined as (33), (12), and (59), respectively. 
Proof. Assume that $V_{n}\left[w_{n}\right]=V_{r, n}\left[w_{n}\right] e^{i n \theta} \mathbf{e}_{r}+V_{\theta, n}\left[w_{n}\right] e^{i n \theta} \mathbf{e}_{\theta}$ is a solution to (48). Then the no-slip boundary condition on $V_{n}\left[w_{n}\right]$ implies

$$
0=\left.V_{\theta, n}\left[w_{n}\right]\right|_{r=1}=-\left.\left(\frac{\mathrm{d}}{\mathrm{d}} \psi_{n}\left[w_{n}\right]\right)\right|_{r=1}=-\int_{1}^{\infty} s^{1-|n|} w_{n}(s) \mathrm{d} s=-d_{n}\left[w_{n}\right],
$$

which is exactly (60) by the definition of $w_{n}$ in (56). Conversely, if (60) holds then the no-slip boundary condition $V_{n}\left[w_{n}\right]=0$ on $\partial \Omega$ is satisfied. Since $f_{n}$ belongs to $C_{0, \sigma}^{\infty}(\Omega)$ the definition of $w_{n}$ in (56) implies that $w_{n}$ is smooth and decays exponentially at spatial infinity. Then the velocity $V_{n}\left[w_{n}\right]$ belongs to $W^{k, 2}(\Omega)^{2} \cap L_{\sigma}^{2}(\Omega)$ for any $k \in \mathbb{N}$ and also to $W_{0}^{1,2}(\Omega)^{2}$ by the fact $V_{n}\left[w_{n}\right]=0$ on $\partial \Omega$. Therefore, $V_{n}\left[w_{n}\right]$ belongs to $D_{L_{\sigma}^{2}}\left(A_{\alpha}\right) \cap$ $W^{k, 2}(\Omega)^{2}$ for any $k \in \mathbb{N}$. Set $w=w_{n} e^{i n \theta}$. Then we observe from rot $V_{n}\left[w_{n}\right]=w$ that

$$
\begin{gathered}
\operatorname{rot}\left(\lambda V_{n}\left[w_{n}\right]-\Delta V_{n}\left[w_{n}\right]+\alpha U^{\perp} \operatorname{rot} V_{n}\left[w_{n}\right]-f\right) \\
=\lambda w-\Delta w+\alpha U \cdot \nabla w-\operatorname{rot} f=0 \quad \text { in } \Omega,
\end{gathered}
$$

for $w_{n}$ is the solution to (51). By Corollary 2.7 there is $p \in \hat{W}^{1,2}(\Omega)$ such that

$$
\lambda V_{n}\left[w_{n}\right]-\Delta V_{n}\left[w_{n}\right]+\alpha U^{\perp} \operatorname{rot} V_{n}\left[w_{n}\right]-f=-\nabla p \quad \text { in } \Omega .
$$

Acting the Helmholtz projection $\mathbb{P}$ on both sides of the above equation, we obtain the equality $\lambda V_{n}\left[w_{n}\right]+A_{\alpha} V_{n}\left[w_{n}\right]=f$. The proof is complete.

The next proposition gives a characterization of the discrete spectrum of $-A_{\alpha}$.

Proposition 3.2 The discrete spectrum of $-\left.A_{\alpha}\right|_{\mathcal{P}_{n} L_{\sigma}^{2}}$ with $|n| \geq 1$ and of $-A_{\alpha}$ respectively satisfy

$$
\sigma_{\text {disc }}\left(-\left.A_{\alpha}\right|_{\mathcal{P}_{n} L_{\sigma}^{2}}\right) \backslash \overline{\mathbb{R}_{-}}=\left\{\lambda \in \mathbb{C} \backslash \overline{\mathbb{R}_{-}} \mid F_{n}(\sqrt{\lambda} ; \alpha)=0\right\},
$$

and

$$
\sigma_{\text {disc }}\left(-A_{\alpha}\right)=\left\{\lambda \in \mathbb{C} \backslash \overline{\mathbb{R}_{-}} \mid F_{n}(\sqrt{\lambda} ; \alpha)=0 \text { for some } n \in \mathbb{Z} \backslash\{0\}\right\} .
$$

Moreover, the eigenspace $E_{\alpha}(\lambda)$ for the eigenvalue $\lambda \in \sigma_{\text {disc }}\left(-A_{\alpha}\right)$ is spanned by

$$
\left\{V_{n, \lambda, \alpha}\right\}_{n \in Z_{\alpha}(\lambda)}, \quad Z_{\alpha}(\lambda)=\left\{n \in \mathbb{Z} \backslash\{0\} \mid F_{n}(\sqrt{\lambda} ; \alpha)=0\right\},
$$

where $V_{n, \lambda, \alpha}=V_{n}\left[K_{\mu_{n}(\alpha)}(\sqrt{\lambda} \cdot)\right]$ is defined as (34) with $w_{n}(r)=K_{\mu_{n}(\alpha)}(\sqrt{\lambda} r)$

Proof. Firstly we assume that $v \in D_{L_{\sigma}^{2}}\left(A_{\alpha}\right) \cap \mathcal{P}_{n} L_{\sigma}^{2}(\Omega)$ satisfies the homogeneous equation

$$
\lambda v+A_{\alpha} v=0,
$$

for some $\lambda \in \mathbb{C} \backslash \overline{\mathbb{R}_{-}}$. Since $A_{\alpha} v=A v+\alpha \mathbb{P}\left(U^{\perp} \operatorname{rot} v\right)$ and $U^{\perp}$ is smooth and bounded in $\Omega$, we have $\operatorname{rot} v \in W^{2,2}(\Omega)$ by the elliptic regularity of the Stokes operator. Taking rot in (64), we obtain the equation (49) with $f=0$ for the vorticity $w=\operatorname{rot} v$. Writing in the polar coordinates $w=w_{n} e^{i n \theta}$, we derive the ordinary differential equation (51) for $w_{n}$ with $h_{n}=0$, and hence, from (56) we have

$$
w_{n}(r)=c_{n} K_{\mu_{n}(\alpha)}(\sqrt{\lambda} r)
$$


for some constant $c_{n}$. In particular, $w_{n}$ decays exponentially as $r \rightarrow \infty$. Then Proposition 2.6 implies the Biot-Savrt law

$$
\begin{aligned}
v & =c_{n} V_{n}\left[K_{\mu_{n}(\alpha)}(\sqrt{\lambda} \cdot)\right] \\
& =c_{n} V_{r, n}\left[K_{\mu_{n}(\alpha)}(\sqrt{\lambda} \cdot)\right] e^{i n \theta} \mathbf{e}_{r}+c_{n} V_{\theta, n}\left[K_{\mu_{n}(\alpha)}(\sqrt{\lambda} \cdot)\right] e^{i n \theta} \mathbf{e}_{\theta},
\end{aligned}
$$

where $V_{n}, V_{r, n}, V_{\theta, n}$ are defined as (34). Let us recall that the no-slip boundary condition $v=0$ on $\partial \Omega$ requires the condition $\left.c_{n} V_{\theta, n}\left[K_{\mu_{n}(\alpha)}(\sqrt{\lambda} \cdot)\right]\right|_{r=1}=0$, which is nothing but the condition

$$
c_{n} F_{n}(\sqrt{\lambda} ; \alpha)=0 .
$$

Therefore, if $\lambda \in \mathbb{C} \backslash \overline{\mathbb{R}_{-}}$satisfies $F_{n}(\sqrt{\lambda} ; \alpha) \neq 0$ then $c_{n}=0$, which gives $v=0$ by (66). Thus $\lambda+\left.A_{\alpha}\right|_{\mathcal{P}_{n} L_{\sigma}^{2}}$ in $\mathcal{P}_{n} L_{\sigma}^{2}(\Omega)$ is one to one in this case, and we conclude that $\lambda \notin \sigma_{\text {disc }}\left(-\left.A_{\alpha}\right|_{\mathcal{P}_{n} L_{\sigma}^{2}}\right)$. Conversely, if $F_{n}(\sqrt{\lambda} ; \alpha)=0$ for some $n \in \mathbb{Z} \backslash\{0\}$ then $V_{n}\left[K_{\mu_{n}(\alpha)}(\sqrt{\lambda} \cdot)\right]$ is a nontrivial solution to (64) by Lemma 3.1. In particular, $\lambda$ is an eigenvalue of $-\left.A_{\alpha}\right|_{\mathcal{P}_{n} L_{\sigma}^{2}}$, which yields $\lambda \in \sigma_{\text {disc }}\left(-\left.A_{\alpha}\right|_{\mathcal{P}_{n} L_{\sigma}^{2}(\Omega)}\right)$ by Proposition 2.12 . Hence we obtain (61), and then also (62) by Proposition 2.12. Next assume $\lambda \in \sigma_{\text {disc }}\left(-A_{\alpha}\right)$ and let $v \in D_{L_{\sigma}^{2}}\left(A_{\alpha}\right)$ be the associated eigenfunction. We have $\lambda \notin \overline{\mathbb{R}_{-}}$by (62). Since the multiplicity of $\lambda$ is finite, $\mathcal{P}_{n} v$ becomes nontrivial only for a finite number of $n \in \mathbb{Z} \backslash\{0\}$ satisfying $F_{n}(\sqrt{\lambda} ; \alpha)=0$, and each $\mathcal{P}_{n} v$ must be a constant multiple of $V_{n}\left[K_{\mu_{n}(\alpha)}(\sqrt{\lambda} \cdot)\right]$ by (66). Collecting these facts, we conclude that the eigenspace $E_{\alpha}(\lambda)$ is spanned by (63). The proof is complete.

Next we consider the possible order of each zero point of $F_{n}(\sqrt{\lambda} ; \alpha)$, which is related with the order of the poles of $\left(\lambda+A_{\alpha}\right)^{-1}$.

Proposition 3.3 Let $\lambda \in \mathbb{C} \backslash \overline{\mathbb{R}_{-}}$be a zero point of $F_{n}(\sqrt{\cdot} ; \alpha)$ such that $\frac{\mathrm{d} F_{n}}{\mathrm{~d} z}(\sqrt{\lambda} ; \alpha)=0$. Then $\frac{\mathrm{d}^{2} F_{n}}{\mathrm{~d} z^{2}}(\sqrt{\lambda} ; \alpha) \neq 0$ and $\lambda \in\left\{z \in C \backslash \overline{\mathbb{R}_{-}}|| \Im(z)|<-| \frac{\alpha}{n} \mid \Re(z)\right\}$. Therefore, $\lambda$ is a zero point of $F_{n}(\sqrt{\cdot} ; \alpha)$ of order at most two, and moreover, of order one if $\lambda \in\{z \in$ $\left.C \backslash \overline{\mathbb{R}_{-}}|| \Im(z)|\geq-| \frac{\alpha}{n} \mid \Re(z)\right\}$.

Proof. For simplicity of notations let us write $F_{n}(z)$ for $F_{n}(z ; \alpha)$ and $\mu_{n}$ for $\mu_{n}(\alpha)$. If $\Re(z)>0$ then we have

$$
\begin{aligned}
\frac{\mathrm{d} F_{n}}{\mathrm{~d} z}(z)=\int_{1}^{\infty} s^{1-|n|} \frac{\mathrm{d}}{\mathrm{d} z}\left(K_{\mu_{n}}(s z)\right) \mathrm{d} s & =\int_{1}^{\infty} s^{2-|n|} \frac{\mathrm{d} K_{\mu_{n}}}{\mathrm{~d} z}(s z) \mathrm{d} s \\
& =\frac{1}{z} \int_{1}^{\infty} s^{2-|n|} \frac{\mathrm{d}}{\mathrm{d} s}\left(K_{\mu_{n}}(s z)\right) \mathrm{d} s \\
& =-\frac{1}{z} K_{\mu_{n}}(z)+\frac{|n|-2}{z} F_{n}(z) .
\end{aligned}
$$

Hence, if $F_{n}\left(z_{0}\right)=\frac{\mathrm{d} F_{n}}{\mathrm{~d} z}\left(z_{0}\right)=0$, where $z_{0}=\sqrt{\lambda}$, then $K_{\mu_{n}}\left(z_{0}\right)=0$. If $\frac{\mathrm{d}^{2} F_{n}}{\mathrm{~d} z^{2}}\left(z_{0}\right)=0$ in addition, then $\frac{\mathrm{d} K_{\mu_{n}}}{\mathrm{~d} z}\left(z_{0}\right)$ must be 0 due to (68), which contradicts with (55). Thus, $\frac{\mathrm{d}^{2} F_{n}}{\mathrm{~d} z^{2}}\left(z_{0}\right) \neq 0$. Next we set $\omega_{n}(r)=K_{\mu_{n}}(\sqrt{\lambda} r)$, which satisfies $\omega_{n}(1)=K_{\mu_{n}}(\sqrt{\lambda})=0$ as is seen in the above argument. Then $\omega_{n}$ is a solution to (51) with $h_{n}=0$ which satisfies the Dirichlet condition $\omega_{n}(1)=0$ and decays exponentially as $r \rightarrow \infty$. Multiplying both sides of (51) (for $h_{n}=0$ ) with $r \bar{\omega}_{n}$, we have from the integration by parts,

$$
\left\|\omega_{n}^{\prime}\right\|^{2}+\lambda\left\|\omega_{n}\right\|^{2}+\left(n^{2}+i \alpha n\right)\left\|\frac{\omega_{n}}{r}\right\|^{2}=0, \quad\left\|\omega_{n}\right\|^{2}=\int_{1}^{\infty}\left|\omega_{n}(r)\right|^{2} r \mathrm{~d} r .
$$


Taking the imaginary part of (69), we obtain

$$
\left\|\frac{\omega_{n}}{r}\right\|^{2}=-\frac{\Im(\lambda)}{\alpha n}\left\|\omega_{n}\right\|^{2},
$$

and thus, we conclude that $-\frac{\Im(\lambda)}{\alpha n}=\left|\frac{\Im(\lambda)}{\alpha n}\right|$. Then the real part of (69) is written as

$$
\left(\Re(\lambda)+\left|\frac{n}{\alpha}\right||\Im(\lambda)|\right)\left\|\omega_{n}\right\|^{2}+\left\|\omega_{n}^{\prime}\right\|^{2}=0 .
$$

Since $\omega_{n}$ is not a constant function, we must have $\Re(\lambda)+\left|\frac{n}{\alpha}\right||\Im(\lambda)|<0$. The proof is complete.

\subsection{Structure of resolvent in $\mathcal{P}_{n} L_{\sigma}^{2}(\Omega)$}

In this section we study a basic structure of the resolvent $\left(\lambda+\left.A_{\alpha}\right|_{\mathcal{P}_{n} L_{\sigma}^{2}}\right)^{-1}$ in $\mathcal{P}_{n} L_{\sigma}^{2}(\Omega)$, which is useful to analyze its quantitative properties.

Proposition 3.4 Let $n \in \mathbb{Z} \backslash\{0\}$. Then there are locally bounded (operator-valued) functions $T_{n, \alpha}, R_{n, \alpha}: \mathbb{C} \backslash \overline{\mathbb{R}_{-}} \rightarrow \mathcal{L}\left(\mathcal{P}_{n} L_{\sigma}^{2}(\Omega)\right)$ such that

$$
\left(\lambda+\left.A_{\alpha}\right|_{\mathcal{P}_{n} L_{\sigma}^{2}}\right)^{-1}=\frac{1}{F_{n}(\sqrt{\lambda} ; \alpha)} T_{n, \alpha}(\lambda)+R_{n, \alpha}(\lambda), \quad \lambda \in \rho\left(-\left.A_{\alpha}\right|_{\mathcal{P}_{n} L_{\sigma}^{2}}\right) \backslash \overline{\mathbb{R}_{-}} .
$$

Proof. Let $f=f_{n} \in \mathcal{P}_{n} C_{0, \sigma}^{\infty}(\Omega)$. Set $w_{n}$ as in (56) for some constant $c_{n}$, which is a smooth and exponentially decaying function solving (51) for $r>1$. We take $c_{n}$ so that (60) holds, i.e.,

$$
c_{n}=-\frac{1}{F_{n}(\sqrt{\lambda} ; \alpha)} c_{n, \lambda}\left[f_{n}\right]
$$

which is well-defined since $F_{n}(\sqrt{\lambda} ; \alpha) \neq 0$ by the assumption $\lambda \in \rho\left(-\left.A_{\alpha}\right|_{\mathcal{P}_{n} L_{\sigma}^{2}}\right) \backslash \overline{\mathbb{R}_{-}}$ and Proposition 3.2. By the choice of $c_{n}$ in (73), Lemma 3.1 implies that $V_{n}\left[w_{n}\right]$ belongs to $D_{L_{\sigma}^{2}}\left(\left.A_{\alpha}\right|_{\mathcal{P}_{n} L_{\sigma}^{2}}\right)=W^{2,2}(\Omega)^{2} \cap W_{0}^{1,2}(\Omega)^{2} \cap \mathcal{P}_{n} L_{\sigma}^{2}(\Omega)$ and satisfies (48) for $f=f_{n}$. Thus we have from (56), with the notation $\mu_{n}=\mu_{n}(\alpha)$,

$$
\begin{aligned}
\left(\lambda+\left.A_{\alpha}\right|_{\mathcal{P}_{n} L_{\sigma}^{2}}\right)^{-1} f_{n} & =V_{n}\left[w_{n}\right] \\
& =c_{n} V_{n}\left[K_{\mu_{n}}(\sqrt{\lambda} \cdot)\right]+V_{n}\left[\Phi_{n, \lambda}\left[f_{n}\right]\right] \\
& =-\frac{c_{n, \lambda}\left[f_{n}\right]}{F_{n}(\sqrt{\lambda} ; \alpha)} V_{n}\left[K_{\mu_{n}}(\sqrt{\lambda} \cdot)\right]+V_{n}\left[\Phi_{n, \lambda}\left[f_{n}\right]\right] .
\end{aligned}
$$

Taking (74) into account, we set

$$
\begin{aligned}
& T_{n, \alpha}(\lambda) f_{n}=-c_{n, \lambda}\left[f_{n}\right] V_{n, \lambda, \alpha}, \quad V_{n, \lambda, \alpha}=V_{n}\left[K_{\mu_{n}}(\sqrt{\lambda} \cdot)\right], \\
& R_{n, \alpha}(\lambda) f_{n}=V_{n}\left[\Phi_{n, \lambda}\left[f_{n}\right]\right] .
\end{aligned}
$$

Note that $T_{n, \alpha}(\lambda) f_{n}$ and $R_{n, \alpha}(\lambda) f_{n}$ themselves are well-defined for any $\lambda \in \mathbb{C} \backslash \overline{\mathbb{R}_{-}}$ and $f_{n} \in \mathcal{P}_{n} C_{0, \sigma}^{\infty}(\Omega)$. Now we fix any $f \in \mathcal{P}_{n} L_{\sigma}^{2}(\Omega)$ and take a sequence $\left\{f^{(k)}\right\}_{k=1}^{\infty}$ in $\mathcal{P}_{n} C_{0, \sigma}^{\infty}(\Omega)$ such that $f^{(k)}$ converges to $f$ in $\mathcal{P}_{n} L_{\sigma}^{2}(\Omega)$. As is observed, for each $f^{(k)}$ we have the formula such as

$$
\left(\lambda+\left.A_{\alpha}\right|_{\mathcal{P}_{n} L_{\sigma}^{2}}\right)^{-1} f^{(k)}=\frac{1}{F_{n}(\sqrt{\lambda} ; \alpha)} T_{n, \alpha}(\lambda) f^{(k)}+R_{n, \alpha}(\lambda) f^{(k)} .
$$


By the assumption $\lambda \in \rho\left(-\left.A_{\alpha}\right|_{\mathcal{P}_{n} L_{\sigma}^{2}}\right)$ we see that the left-hand side of (77) converges to $\left(\lambda+\left.A_{\alpha}\right|_{\mathcal{P}_{n} L_{\sigma}^{2}}\right)^{-1} f$ in $\mathcal{P}_{n} L_{\sigma}^{2}(\Omega)$. On the other hand, we can show that for any $\lambda \in \mathbb{C} \backslash \overline{\mathbb{R}_{-}}$ the linear operators $T_{n, \alpha}(\lambda)$ and $R_{n, \alpha}(\lambda)$ are extended to bounded operators from $\mathcal{P}_{n} L_{\sigma}^{2}(\Omega)$ to $\mathcal{P}_{n} L_{\sigma}^{2}(\Omega)$, whose proof requires long calculations and thus is postponed to Section 3.3 below; see Corollary 3.18. Admitting Corollary 3.18, we can take the limit $k \rightarrow \infty$ also in the right-hand side of (77), which converges to $\frac{1}{F_{n}(\sqrt{\lambda} ; \alpha)} T_{n, \alpha}(\lambda) f+R_{n, \alpha}(\lambda) f$ in $\mathcal{P}_{n} L_{\sigma}^{2}(\Omega)$. Since $f \in \mathcal{P}_{n} L_{\sigma}^{2}(\Omega)$ is arbitrary we obtain the formula (72). In Corollary 3.18 we will show that $T_{n, \alpha}, R_{n, \alpha}: \mathbb{C} \backslash \overline{\mathbb{R}_{-}} \rightarrow \mathcal{L}\left(\mathcal{P}_{n} L_{\sigma}^{2}(\Omega)\right)$ are locally bounded. Hence, the proof of Proposition 3.4 is complete by admitting Corollary 3.18 .

Corollary 3.5 If $\lambda_{0} \in \sigma_{\text {disc }}\left(-A_{\alpha}\right)$ then it is a pole of the resolvent $\left(\lambda+A_{\alpha}\right)^{-1}$ of order at most two. Moreover, if $\lambda_{0} \in \sigma_{\text {disc }}\left(-A_{\alpha}\right)$ belongs to the set $\{z \in \mathbb{C}|| \Im(z)|\geq-| \alpha \mid \Re(z)\}$, then $\lambda_{0}$ is a pole of $\left(\lambda+A_{\alpha}\right)^{-1}$ of order one.

Proof. For $\lambda \in \rho\left(-A_{\alpha}\right)$ we have $\lambda \notin \overline{\mathbb{R}_{-}}$by Proposition 2.12 and the decomposition

$$
\begin{aligned}
& \left(\lambda+A_{\alpha}\right)^{-1} \\
& =\left(\lambda+\left.A\right|_{L_{\sigma, \text { rad }}^{2}}\right)^{-1} \oplus\left(\lambda+\left.A_{\alpha}\right|_{\mathcal{Q}_{m} L_{\sigma}^{2}}\right)^{-1} \oplus\left(\oplus_{1 \leq|n| \leq m}\left(\lambda+\left.A_{\alpha}\right|_{\mathcal{P}_{n} L_{\sigma}^{2}}\right)^{-1}\right),
\end{aligned}
$$

holds for each $m \in \mathbb{N}$ by Proposition 2.10. On the other hand, by Proposition 3.2 if $\lambda_{0} \in$ $\sigma_{\text {disc }}\left(-A_{\alpha}\right)$ then $\lambda_{0} \notin \overline{\mathbb{R}_{-}}$and there is $n \in \mathbb{Z} \backslash\{0\}$ such that $F_{n}\left(\sqrt{\lambda_{0}} ; \alpha\right)=0$ and $V_{n}\left[K_{\mu_{n}(\alpha)}\left(\sqrt{\lambda_{0}} \cdot\right)\right]$ is an eigenfunction. In particular, the number of such $n$ is finite since the multiplicity of $\lambda_{0}$ is finite. Hence, if we take $m$ large enough, then $\lambda_{0}$ must belong to $\rho\left(-\left.A\right|_{L_{\sigma, \text { rad }}^{2}}\right) \cap \rho\left(-\left.A_{\alpha}\right|_{\mathcal{Q}_{m} L_{\sigma}^{2}}\right)$ and the singularity of $\left(\lambda+A_{\alpha}\right)^{-1}$ at $\lambda=\lambda_{0}$ arises only from a finite number of $\left(\lambda+\left.A_{\alpha}\right|_{\mathcal{P}_{n} L_{\sigma}^{2}}\right)^{-1}$ such that $1 \leq|n| \leq m$. Therefore, it suffices to consider the possible singularity of $\left(\lambda+\left.A_{\alpha}\right|_{\mathcal{P}_{n} L_{\sigma}^{2}}\right)^{-1}$ in $\mathbb{C} \backslash \overline{\mathbb{R}_{-}}$for each $n \in \mathbb{Z} \backslash\{0\}$. Note that $\sigma_{\text {disc }}\left(-\left.A_{\alpha}\right|_{\mathcal{P}_{n} L_{\sigma}^{2}}\right)$ consists of the poles of $\left(\lambda+\left.A_{\alpha}\right|_{\mathcal{P}_{n} L_{\sigma}^{2}}\right)^{-1}$. Then Proposition 3.4 implies that the order of any pole $\lambda_{0} \in \mathbb{C} \backslash \overline{\mathbb{R}_{-}}$of $\left(\lambda+\left.A_{\alpha}\right|_{\mathcal{P}_{n} L_{\sigma}^{2}}\right)^{-1}$ is less than or equal to the order of the zero point $\lambda_{0}$ of $F_{n}(\sqrt{\lambda} ; \alpha)$. Hence we conclude from Proposition 3.3 that $\lambda_{0}$ is a pole of $\left(\lambda+\left.A_{\alpha}\right|_{\mathcal{P}_{n} L_{\sigma}^{2}}\right)^{-1}$ of order at most two, and moreover, of order one in the case $\lambda_{0} \in\left\{z \in \mathbb{C}|| \Im(z)|\geq-| \frac{\alpha}{n} \mid \Re(z)\right\}$. The proof is complete.

Proof of (1), (2), (3) in Theorem 1.5. The statements (1) and (2) follow from Propositions 2.12 and 3.2. The statement (3) is proved in Corollary 3.5. The proof is complete.

The proof of the statement (4) in Theorem 1.5 requires detailed computations, which will be given in Section 3.6 below.

\subsection{Resolvent estimates in $\mathcal{P}_{n} L_{\sigma}^{2}(\Omega)$}

In this section we will establish the estimates of the resolvent $\left(\lambda+\left.A_{\alpha}\right|_{\mathcal{P}_{n} L_{\sigma}^{2}}\right)^{-1}$. As is seen in (74), we have the formula

$$
\left(\lambda+\left.A_{\alpha}\right|_{\mathcal{P}_{n} L_{\sigma}^{2}}\right)^{-1} f_{n}=-\frac{c_{n, \lambda}\left[f_{n}\right]}{F_{n}(\sqrt{\lambda} ; \alpha)} V_{n}\left[K_{\mu_{n}}(\sqrt{\lambda} \cdot)\right]+V_{n}\left[\Phi_{n, \lambda}\left[f_{n}\right]\right]
$$

for $\lambda \in \rho\left(-\left.A_{\alpha}\right|_{\mathcal{P}_{n} L_{\sigma}^{2}}\right) \backslash \overline{\mathbb{R}_{-}}$and $f_{n} \in \mathcal{P}_{n} L_{\sigma}^{2}(\Omega)$, where $c_{n, \lambda}\left[f_{n}\right]$ is defined as (59) and $V_{n}\left[\Phi_{n, \lambda}\left[f_{n}\right]\right]$ is defined as (34) and (58). The main results of this section are stated as Theorems 3.19 and 3.23. 


\subsection{1 $L^{p}-L^{q}$ estimates for velocity}

For the moment let us focus on the estimates of the term $V_{n}\left[\Phi_{n, \lambda}\left[f_{n}\right]\right]$ in (79). In view of the definition of the operator $V_{n}$ in (33) - (34), we need to establish the estimates of the following terms:

$$
\frac{1}{r^{|n|}} \int_{1}^{r} s^{1+|n|} \Phi_{n, \lambda}\left[f_{n}\right](s) \mathrm{d} s, \quad r^{|n|} \int_{r}^{\infty} s^{1-|n|} \Phi_{n, \lambda}\left[f_{n}\right](s) \mathrm{d} s .
$$

Motivated by the definition of $\Phi_{n, \lambda}\left[f_{n}\right]$ in (58), we set for $f_{n}=f_{r, n} e^{i n \theta} \mathbf{e}_{r}+f_{\theta, n} e^{i n \theta} \mathbf{e}_{\theta}$,

$$
j_{n}=\mu f_{\theta, n}-i n f_{r, n}, \quad g_{n}=\mu f_{\theta, n}+i n f_{r, n} .
$$

In order to derive the precise estimates for both cases $|\lambda| \rightarrow 0$ and $|\lambda| \rightarrow \infty$ it is important to rewrite (80) in a suitable manner. To this end let us start from the following

Lemma 3.6 Let $f_{n} \in \mathcal{P}_{n} C_{0}^{\infty}(\Omega)^{2}$. Then we have

$$
\frac{1}{r^{|n|}} \int_{1}^{r} s^{1+|n|} \Phi_{n, \lambda}\left[f_{n}\right](s) \mathrm{d} s=\sum_{l=1}^{9} J_{l}\left[f_{n}\right](r),
$$

where

$$
\begin{aligned}
& J_{1}\left[f_{n}\right](r)=-\frac{1}{r^{|n|}} \int_{1}^{r} \int_{\tau}^{r} s^{1+|n|} K_{\mu_{n}}(\sqrt{\lambda} s) \mathrm{d} s I_{\mu_{n}}(\sqrt{\lambda} \tau) g_{n} \mathrm{~d} \tau \\
& J_{2}\left[f_{n}\right](r)=-\frac{\mu_{n}+|n|}{r^{|n|}} \int_{1}^{r} \int_{\tau}^{r} s^{|n|} K_{\mu_{n}-1}(\sqrt{\lambda} s) \mathrm{d} s I_{\mu_{n}+1}(\sqrt{\lambda} \tau) f_{\theta, n} \tau \mathrm{d} \tau \\
& J_{3}\left[f_{n}\right](r)=\frac{1}{r^{|n|}} \int_{1}^{r} \int_{1}^{\tau} s^{1+|n|} I_{\mu_{n}}(\sqrt{\lambda} s) \mathrm{d} s K_{\mu_{n}}(\sqrt{\lambda} \tau) j_{n} \mathrm{~d} \tau \\
& J_{4}\left[f_{n}\right](r)=\frac{\mu_{n}-|n|}{r^{|n|}} \int_{1}^{r} \int_{1}^{\tau} s^{|n|} I_{\mu_{n}+1}(\sqrt{\lambda} s) \mathrm{d} s K_{\mu_{n}-1}(\sqrt{\lambda} \tau) f_{\theta, n} \tau \mathrm{d} \tau \\
& J_{5}\left[f_{n}\right](r)=\frac{1}{r^{|n|}} \int_{1}^{r} s^{1+|n|} I_{\mu_{n}}(\sqrt{\lambda} s) \mathrm{d} s \int_{r}^{\infty} K_{\mu_{n}}(\sqrt{\lambda} s) j_{n} \mathrm{~d} s \\
& J_{6}\left[f_{n}\right](r)=\frac{\mu_{n}-|n|}{r^{|n|}} \int_{1}^{r} s^{|n|} I_{\mu_{n}+1}(\sqrt{\lambda} s) \mathrm{d} s \int_{r}^{\infty} K_{\mu_{n}-1}(\sqrt{\lambda} s) f_{\theta, n} s \mathrm{~d} s \\
& J_{7}\left[f_{n}\right](r)=r K_{\mu_{n}-1}(\sqrt{\lambda} r) \int_{1}^{r} I_{\mu_{n}+1}(\sqrt{\lambda} \tau) f_{\theta, n} \tau \mathrm{d} \tau \\
& J_{8}\left[f_{n}\right](r)=r I_{\mu_{n}+1}(\sqrt{\lambda} r) \int_{r}^{\infty} K_{\mu_{n}-1}(\sqrt{\lambda} \tau) f_{\theta, n} \tau \mathrm{d} \tau \\
& J_{9}\left[f_{n}\right](r)=-\frac{1}{r^{|n|}} I_{\mu_{n}+1}(\sqrt{\lambda}) \int_{1}^{\infty} K_{\mu_{n}-1}(\sqrt{\lambda} \tau) f_{\theta, n} \tau \mathrm{d} \tau
\end{aligned}
$$


Proof. By the definition of $\Phi_{n, \lambda}\left[f_{n}\right]$ in (58), we have

$$
\begin{aligned}
\frac{1}{r^{|n|}} \int_{1}^{r} s^{1+|n|} \Phi_{n, \lambda}\left[f_{n}\right](s) \mathrm{d} s= & -\frac{1}{r^{|n|}} \int_{1}^{r} s^{1+|n|} K_{\mu_{n}}(\sqrt{\lambda} s) \int_{1}^{s} I_{\mu_{n}}(\sqrt{\lambda} \tau) g_{n} \mathrm{~d} \tau \mathrm{d} s \\
& -\frac{1}{r^{|n|}} \int_{1}^{r} s^{1+|n|} \sqrt{\lambda} K_{\mu_{n}}(\sqrt{\lambda} s) \int_{1}^{s} I_{\mu_{n}+1}(\sqrt{\lambda} \tau) f_{\theta, n} \tau \mathrm{d} \tau \mathrm{d} s \\
& +\frac{1}{r^{|n|}} \int_{1}^{r} s^{1+|n|} I_{\mu_{n}}(\sqrt{\lambda} s) \int_{s}^{\infty} K_{\mu_{n}}(\sqrt{\lambda} \tau) j_{n} \mathrm{~d} \tau \mathrm{d} s \\
& +\frac{1}{r^{|n|}} \int_{1}^{r} s^{1+|n|} \sqrt{\lambda} I_{\mu_{n}}(\sqrt{\lambda} s) \int_{s}^{\infty} K_{\mu_{n}-1}(\sqrt{\lambda} \tau) f_{\theta, n} \tau \mathrm{d} \tau \mathrm{d} s \\
= & I_{1}+I_{2}+I_{3}+I_{4} .
\end{aligned}
$$

By changing the order of the integration we have $I_{1}=J_{1}\left[f_{n}\right]$. Similarly, we have for $I_{2}$,

$$
I_{2}=-\frac{1}{r^{|n|}} \int_{1}^{r} \int_{\tau}^{r} s^{1+|n|} \sqrt{\lambda} K_{\mu_{n}}(\sqrt{\lambda} s) \mathrm{d} s I_{\mu_{n}+1}(\sqrt{\lambda} \tau) f_{\theta, n} \tau \mathrm{d} \tau
$$

Then the identity $\frac{\mathrm{d} K_{\mu-1}}{\mathrm{~d} z}(z)=\frac{\mu-1}{z} K_{\mu-1}(z)-K_{\mu}(z)$ in (327) implies that

$$
\sqrt{\lambda} K_{\mu_{n}}(\sqrt{\lambda} s)=\frac{\mu_{n}-1}{s} K_{\mu_{n}-1}(\sqrt{\lambda} s)-\frac{\mathrm{d}}{\mathrm{d} s} K_{\mu_{n}-1}(\sqrt{\lambda} s),
$$

which yields from the integration by parts,

$$
\begin{aligned}
I_{2} & =-\frac{\mu_{n}+|n|}{r^{|n|}} \int_{1}^{r} \int_{\tau}^{r} s^{|n|} K_{\mu_{n}-1}(\sqrt{\lambda} s) \mathrm{d} s I_{\mu_{n}+1}(\sqrt{\lambda} \tau) f_{\theta, n} \tau \mathrm{d} \tau \\
& +\frac{1}{r^{|n|}} \int_{1}^{r}\left\{r^{1+|n|} K_{\mu_{n}-1}(\sqrt{\lambda} r)-\tau^{1+|n|} K_{\mu_{n}-1}(\sqrt{\lambda} \tau)\right\} I_{\mu_{n}+1}(\sqrt{\lambda} \tau) f_{\theta, n} \tau \mathrm{d} \tau \\
& =J_{2}\left[f_{n}\right]+J_{7}\left[f_{n}\right]-\frac{1}{r^{|n|}} \int_{1}^{r} \tau^{1+|n|} K_{\mu_{n}-1}(\sqrt{\lambda} \tau) I_{\mu_{n}+1}(\sqrt{\lambda} \tau) f_{\theta, n} \tau \mathrm{d} \tau
\end{aligned}
$$

Next we consider $I_{3}$. By decomposing the integral $\int_{s}^{\infty}$ into $\int_{s}^{r}+\int_{r}^{\infty}$ and by changing the order of the integration $\int_{1}^{r} \int_{s}^{r}$ we have

$$
I_{3}=J_{3}\left[f_{n}\right]+J_{5}\left[f_{n}\right]
$$

Similarly, we have for $I_{4}$,

$$
\begin{aligned}
I_{4} & =\frac{1}{r^{|n|}} \int_{1}^{r} \int_{1}^{\tau} s^{1+|n|} \sqrt{\lambda} I_{\mu_{n}}(\sqrt{\lambda} s) \mathrm{d} s K_{\mu_{n}-1}(\sqrt{\lambda} \tau) f_{\theta, n} \tau \mathrm{d} \tau \\
& +\frac{1}{r^{|n|}} \int_{1}^{r} s^{1+|n|} \sqrt{\lambda} I_{\mu_{n}}(\sqrt{\lambda} s) \mathrm{d} s \int_{r}^{\infty} K_{\mu_{n}-1}(\sqrt{\lambda} s) f_{\theta, n} s \mathrm{~d} s .
\end{aligned}
$$

Then we recall the identity $\frac{\mathrm{d} I_{\mu+1}}{\mathrm{~d} z}(z)=-\frac{\mu+1}{z} I_{\mu+1}(z)+I_{\mu}(z)$ in (324), which leads to

$$
\sqrt{\lambda} I_{\mu_{n}}(\sqrt{\lambda} s)=\frac{\mu_{n}+1}{s} I_{\mu_{n}+1}(\sqrt{\lambda} s)+\frac{\mathrm{d}}{\mathrm{d} s} I_{\mu_{n}+1}(\sqrt{\lambda} s) .
$$


Hence by the integration by parts the term $I_{4}$ is written as

$$
\begin{aligned}
I_{4}= & \frac{\mu_{n}-|n|}{r^{|n|}} \int_{1}^{r} \int_{1}^{\tau} s^{|n|} I_{\mu_{n}+1}(\sqrt{\lambda} s) \mathrm{d} s K_{\mu_{n}-1}(\sqrt{\lambda} \tau) f_{\theta, n} \tau \mathrm{d} \tau \\
+ & \frac{1}{r^{|n|}} \int_{1}^{r}\left\{\tau^{1+|n|} I_{\mu_{n}+1}(\sqrt{\lambda} \tau)-I_{\mu_{n}+1}(\sqrt{\lambda})\right\} K_{\mu_{n}-1}(\sqrt{\lambda} \tau) f_{\theta, n} \tau \mathrm{d} \tau \\
+ & \frac{\mu_{n}-|n|}{r^{|n|}} \int_{1}^{r} s^{|n|} I_{\mu_{n}+1}(\sqrt{\lambda} s) \mathrm{d} s \int_{r}^{\infty} K_{\mu_{n}-1}(\sqrt{\lambda} s) f_{\theta, n} s \mathrm{~d} s \\
+ & \frac{1}{r^{|n|}}\left\{r^{1+|n|} I_{\mu_{n}+1}(\sqrt{\lambda} r)-I_{\mu_{n}+1}(\sqrt{\lambda})\right\} \int_{r}^{\infty} K_{\mu_{n}-1}(\sqrt{\lambda} \tau) f_{\theta, n} \tau \mathrm{d} \tau \\
= & J_{4}\left[f_{n}\right]+J_{6}\left[f_{n}\right]+J_{8}\left[f_{n}\right]+J_{9}\left[f_{n}\right] \\
& +\frac{1}{r^{|n|}} \int_{1}^{r} \tau^{1+|n|} I_{\mu_{n}+1}(\sqrt{\lambda} \tau) K_{\mu_{n}-1}(\sqrt{\lambda} \tau) f_{\theta, n} \tau \mathrm{d} \tau .
\end{aligned}
$$

Collecting these, we obtain (82). The proof is complete.

The next lemma gives the estimates of each operator $J_{l}, l=1, \cdots, 9$ in Lemma 3.6. The main tools of the proof are the pointwise estimates of the modified Bessel functions stated in Section A, and what is essential here is the condition $\Re\left(\mu_{n}\right)>|n|$ for the order of the Bessel functions $I_{\mu_{n}}$ and $K_{\mu_{n}}$. This condition is satisfied due to the definition $\mu_{n}=$ $\mu_{n}(\alpha)=\left(n^{2}+i \alpha n\right)^{1 / 2}$. In the lemmas below we will establish the estimates of $J_{l}$ under the condition $\Re(\mu)>|n|$, rather than the concrete value of $\mu_{n}(\alpha)$, for generality of the result and for simplicity of notations. Taking the expression $f_{n}=f_{r, n} e^{i n \theta} \mathbf{e}_{r}+f_{\theta, n} e^{i n \theta} \mathbf{e}_{\theta}$ into account, we set for $f_{n} \in \mathcal{P}_{n} C_{0}^{\infty}(\Omega)^{2}$,

$$
\begin{aligned}
\left\|f_{n}\right\|_{L^{q}(\Omega)} & =\left(2 \pi \int_{1}^{\infty}\left(\left|f_{r, n}(r)\right|^{q}+\left|f_{\theta, n}(r)\right|^{q}\right) r \mathrm{~d} r\right)^{\frac{1}{q}}, \quad 1 \leq q<\infty, \\
\left\|f_{n}\right\|_{L^{\infty}(\Omega)} & =\sup _{r>1}\left(\left|f_{r, n}(r)\right|+\left|f_{\theta, n}(r)\right|\right) .
\end{aligned}
$$

Lemma 3.7 Let $n \in \mathbb{Z} \backslash\{0\}$, $\Re(\mu)>|n|$, and let $\lambda \in \Sigma_{\pi-\epsilon}$ for some $\epsilon \in\left(0, \frac{\pi}{2}\right)$. Then there is a positive constant $C=C(\epsilon, n, \mu)$ such that the following statements hold.

(1) Let $l=1, \cdots, 6$, and let $f_{n} \in \mathcal{P}_{n} C_{0}^{\infty}(\Omega)^{2}$. Then we have

$$
\begin{aligned}
& \sup _{r \geq 1} r^{\frac{2}{q}-1}\left|J_{l}\left[f_{n}\right](r)\right| \leq \frac{C}{|\lambda|}\left\|f_{n}\right\|_{L^{q}(\Omega)}, \quad 1 \leq q \leq \infty, \\
& \sup _{r \geq 1} r^{-1}\left|J_{l}\left[f_{n}\right](r)\right| \leq C\left\|f_{n}\right\|_{L^{1}(\Omega)} .
\end{aligned}
$$

(2) Let $l=7,8$, and let $f_{n} \in \mathcal{P}_{n} C_{0}^{\infty}(\Omega)^{2}$. Then we have

$$
\begin{aligned}
\int_{1}^{\infty} & r^{-1}\left|J_{l}\left[f_{n}\right](r)\right| r \mathrm{~d} r \leq \frac{C}{|\lambda|}\left\|f_{n}\right\|_{L^{1}(\Omega)}, \\
& \sup _{r \geq 1} r^{-1}\left|J_{l}\left[f_{n}\right](r)\right| \leq \frac{C}{|\lambda|}\left\|f_{n}\right\|_{L^{\infty}(\Omega)}, \\
& \sup _{r \geq 1} r^{-1}\left|J_{l}\left[f_{n}\right](r)\right| \leq C\left\|f_{n}\right\|_{L^{1}(\Omega)} .
\end{aligned}
$$


(3) Let $l=9$ and let $f_{n} \in \mathcal{P}_{n} C_{0}^{\infty}(\Omega)^{2}$. If $\Re(\sqrt{\lambda}) \geq 1$ then

$$
\begin{aligned}
& \left|J_{9}\left[f_{n}\right](1)\right| \leq C|\lambda|^{-\frac{1}{2}}\left\|f_{n}\right\|_{L^{1}(\Omega)}, \\
& \left|J_{9}\left[f_{n}\right](1)\right| \leq C|\lambda|^{-1}\left\|f_{n}\right\|_{L^{\infty}(\Omega)} .
\end{aligned}
$$

Remark 3.8 (1) Although we can derive the estimate of $J_{9}\left[f_{n}\right](r)$ also for $r>1$ or $\Re(\sqrt{\lambda}) \leq 1$, the estimates (91) and (92) are enough for later use.

(2) We do not need to assume the divergence free condition on $f_{n}$ in Lemma 3.7.

Proof of Lemma 3.7. (1) (i) Estimate of $J_{1}\left[f_{n}\right]$ : If $\Re(\sqrt{\lambda}) \leq r^{-1}$ then (333) and Lemma A. 2 yield

$$
\begin{aligned}
\left|J_{1}\left[f_{n}\right](r)\right| & \leq C r^{-|n|}|\lambda|^{-\frac{\Re(\mu)}{2}} \int_{1}^{r} r^{2} \tau^{|n|-\Re(\mu)}|\sqrt{\lambda} \tau|^{\Re(\mu)}\left|g_{n}\right| \mathrm{d} \tau \\
& \leq C r^{2-|n|} \int_{1}^{r} \tau^{|n|}\left|g_{n}\right| \mathrm{d} \tau .
\end{aligned}
$$

Hence, if $\Re(\sqrt{\lambda}) \leq r^{-1}$ then we have for $l=1$,

$$
\begin{aligned}
r^{\frac{2}{q}-1}\left|J_{l}\left[f_{n}\right](r)\right| & \leq C|\lambda|^{-1}\left\|f_{n}\right\|_{L^{q}(\Omega)}, \quad 1 \leq q \leq \infty, \\
r^{-1}\left|J_{l}\left[f_{n}\right](r)\right| & \leq C\left\|f_{n}\right\|_{L^{1}(\Omega)} .
\end{aligned}
$$

If $\Re(\sqrt{\lambda}) \geq r^{-1}$ then we divide the integral into $\int_{1}^{\max \left\{\frac{1}{\Re(\sqrt{\lambda})}, 1\right\}} \int_{\tau}^{r}$ and $\int_{\max \left\{\frac{1}{\Re(\sqrt{\lambda})}, 1\right\}}^{r} \int_{\tau}^{r}$. As for the first term, it suffices to consider the case $\Re(\sqrt{\lambda}) \leq 1$ and we apply (332) and Lemma A.2, which gives

$$
\begin{aligned}
& r^{-|n|}\left|\int_{1}^{\frac{1}{\Re(\sqrt{\lambda})}} \int_{\tau}^{r}\right| \\
& \leq C r^{-|n|} \int_{1}^{\frac{1}{\Re(\sqrt{\lambda})}}\left(|\lambda|^{-1-\frac{|n|}{2}}+|\lambda|^{-\frac{\Re(\mu)}{2}-1} \tau^{|n|-\Re(\mu)} \mathrm{d} s\right)|\sqrt{\lambda} \tau|^{\Re(\mu)}\left|g_{n}\right| \mathrm{d} \tau \\
& \leq C r^{-|n|}|\lambda|^{-1+\frac{\Re(\mu)-|n|}{2}} \int_{1}^{\frac{1}{\Re(\sqrt{\lambda})}} \tau^{\Re(\mu)}\left|g_{n}\right| \mathrm{d} \tau+C r^{-|n|}|\lambda|^{-1} \int_{1}^{\frac{1}{\Re(\sqrt{\lambda})}} \tau^{|n|}\left|g_{n}\right| \mathrm{d} \tau .
\end{aligned}
$$

Next we have from (331) and Lemma A.3,

$$
\begin{aligned}
r^{-|n|}\left|\int_{\max \left\{\frac{1}{\Re(\sqrt{\lambda})}, 1\right\}}^{r} \int_{\tau}^{r}\right| & \leq C r^{-|n|}|\lambda|^{-\frac{3}{4}} \int_{1}^{r} \tau^{\frac{1}{2}+|n|} e^{-\tau \Re(\sqrt{\lambda})}|\sqrt{\lambda} \tau|^{-\frac{1}{2}} e^{\tau \Re(\sqrt{\lambda})}\left|g_{n}\right| \mathrm{d} \tau \\
& \leq C r^{-|n|}|\lambda|^{-1} \int_{1}^{r} \tau^{|n|}\left|g_{n}\right| \mathrm{d} \tau
\end{aligned}
$$

Collecting (96) and (97), we obtain (94) and (95) also for the case $\Re(\sqrt{\lambda}) \geq r^{-1}$ and $l=1$. The estimate for $J_{1}\left[f_{n}\right]$ has been proved.

(ii) Estimate of $J_{2}\left[f_{n}\right]$ : The proof is the same as in the proof for $J_{1}\left[f_{n}\right]$, and we omit it here.

(iii) Estimate of $J_{3}\left[f_{n}\right]$ : If $\Re(\sqrt{\lambda}) \leq r^{-1}$ then (336) and Lemma A.2 yield

$$
\begin{aligned}
\left|J_{3}\left[f_{n}\right](r)\right| & \leq C r^{-|n|}|\lambda|^{\Re(\mu)} \int_{1}^{r} \tau^{2+|n|+\Re(\mu)}|\sqrt{\lambda} \tau|^{-\Re(\mu)}\left|j_{n}\right| \mathrm{d} \tau \\
& \leq C r^{-|n|} \int_{1}^{r} \tau^{2+|n|}\left|j_{n}\right| \mathrm{d} \tau .
\end{aligned}
$$


Hence, we obtain (94) and (95) for $\Re(\sqrt{\lambda}) \leq r^{-1}$ and $l=3$.

If $\Re(\sqrt{\lambda}) \geq r^{-1}$ then we divide the integral into $\int_{1}^{\max \left\{\frac{1}{\Re(\sqrt{\lambda})}, 1\right\}} \int_{1}^{\tau}$ and $\int_{\max \left\{\frac{1}{\Re(\sqrt{\lambda})}, 1\right\}}^{r} \int_{1}^{\tau}$.

As for the first term, it suffices to consider the case $\Re(\sqrt{\lambda}) \leq 1$ and we apply (336) and Lemma A. 2 as in the derivation of (98), which gives

$$
r^{-|n|}\left|\int_{1}^{\max \left\{\frac{1}{\Re(\sqrt{\lambda})}, 1\right\}} \int_{1}^{\tau}\right| \leq C r^{-|n|} \int_{1}^{\frac{1}{\Re(\sqrt{\lambda})}} \tau^{2+|n|}\left|j_{n}\right| \mathrm{d} \tau, \quad \Re(\sqrt{\lambda}) \leq 1 .
$$

On the other hand, (337) and Lemma A.3 yield

$$
\begin{aligned}
r^{-|n|}\left|\int_{\max \left\{\frac{1}{\Re(\sqrt{\lambda})}, 1\right\}}^{r} \int_{1}^{\tau}\right| & \leq C r^{-|n|}|\lambda|^{-\frac{3}{4}} \int_{1}^{r} \tau^{\frac{1}{2}+|n|} e^{\tau \Re(\sqrt{\lambda})}|\sqrt{\lambda} \tau|^{-\frac{1}{2}} e^{-\tau \Re(\sqrt{\lambda})}\left|j_{n}\right| \mathrm{d} \tau \\
& =C r^{-|n|}|\lambda|^{-1} \int_{1}^{r} \tau^{|n|}\left|j_{n}\right| \mathrm{d} \tau
\end{aligned}
$$

The estimates (99) and (100) imply (94) and (95) for the case $\Re(\sqrt{\lambda}) \geq r^{-1}$ and $l=3$. The estimate for $J_{3}\left[f_{n}\right]$ has been proved.

(iv) Estimate of $J_{4}\left[f_{n}\right]$ : The proof is the same as in the proof for $J_{3}\left[f_{n}\right]$, and we omit it here.

(v) Estimate of $J_{5}\left[f_{n}\right]$ : Firstly we consider the case $\Re(\sqrt{\lambda}) \geq r^{-1}$. Then Lemma A.3 implies

$$
\left|\int_{r}^{\infty} K_{\mu}(\sqrt{\lambda} s) j_{n} \mathrm{~d} s\right| \leq C \int_{r}^{\infty}|\sqrt{\lambda} s|^{-\frac{1}{2}} e^{-s \Re(\sqrt{\lambda})}\left|j_{n}\right| \mathrm{d} s .
$$

Hence, together with (337), we have

$$
\begin{aligned}
\left|J_{5}\left[f_{n}\right](r)\right| & \leq C r^{-|n|}|\lambda|^{-\frac{3}{4}} r^{\frac{1}{2}+|n|} e^{r \Re(\sqrt{\lambda})} \int_{r}^{\infty}|\sqrt{\lambda} s|^{-\frac{1}{2}} e^{-s \Re(\sqrt{\lambda})}\left|j_{n}\right| \mathrm{d} s \\
& \leq C|\lambda|^{-1} r^{\frac{1}{2}} e^{r \Re(\sqrt{\lambda})} \int_{r}^{\infty} s^{-\frac{1}{2}} e^{-s \Re(\sqrt{\lambda})}\left|j_{n}\right| \mathrm{d} s
\end{aligned}
$$

which yields (94) and (95) for $\Re(\sqrt{\lambda}) \geq r^{-1}$ and $l=5$. Next we consider the case $\Re(\sqrt{\lambda}) \leq r^{-1}$. We observe from Lemma A.2 and Lemma A.3 that

$$
\begin{aligned}
& \left|\int_{r}^{\infty} K_{\mu}(\sqrt{\lambda} s) j_{n} \mathrm{~d} s\right| \leq\left|\int_{r}^{\frac{1}{\Re(\sqrt{\lambda})}}\right|+\left|\int_{\frac{1}{\Re(\sqrt{\lambda})}}^{\infty}\right| \\
& \leq C \int_{r}^{\frac{1}{\Re(\sqrt{\lambda})}}|\sqrt{\lambda} s|^{-\Re(\mu)}\left|j_{n}\right| \mathrm{d} s+C \int_{\frac{1}{\Re(\sqrt{\lambda})}}^{\infty}|\sqrt{\lambda} s|^{-\frac{1}{2}} e^{-s \Re(\sqrt{\lambda})}\left|j_{n}\right| \mathrm{d} s .
\end{aligned}
$$

Thus, (336) implies for $\Re(\sqrt{\lambda}) \leq r^{-1}$,

$$
\begin{aligned}
\left|J_{5}\left[f_{n}\right](r)\right| \leq & C r^{-|n|}|\lambda|^{\frac{\Re(\mu)}{2}} r^{2+|n|+\Re(\mu)}\left(\int_{r}^{\frac{1}{\Re(\sqrt{\lambda})}}|\sqrt{\lambda} s|^{-\Re(\mu)}\left|j_{n}\right| \mathrm{d} s\right. \\
& \left.+\int_{\frac{1}{\Re(\sqrt{\lambda})}}^{\infty}|\sqrt{\lambda} s|^{-\frac{1}{2}} e^{-s \Re(\sqrt{\lambda})}\left|j_{n}\right| \mathrm{d} s\right) \\
\leq & C r^{2+\Re(\mu)} \int_{r}^{\frac{1}{\Re(\sqrt{\lambda})}} s^{-\Re(\mu)}\left|j_{n}\right| \mathrm{d} s \\
& +C r^{2+\Re(\mu)}|\lambda|^{\frac{\Re(\mu)}{2}}-\frac{1}{4} \int_{\frac{1}{\Re(\sqrt{\lambda})}}^{\infty} s^{-\frac{1}{2}} e^{-s \Re(\sqrt{\lambda})}\left|j_{n}\right| \mathrm{d} s .
\end{aligned}
$$


Then the direct calculation shows that (104) gives (94) and (95) for the case $\Re(\sqrt{\lambda}) \leq r^{-1}$ and $l=5$. The estimate for $J_{5}\left[f_{n}\right]$ has been proved.

(vi) Estimate of $J_{6}\left[f_{n}\right]$ : The proof is the same as in the proof for $J_{5}\left[f_{n}\right]$, and we omit it here.

(2) Estimate of $J_{7}\left[f_{n}\right]$ and $J_{8}\left[f_{n}\right]$ : We give a proof of the estimates only for $J_{7}\left[f_{n}\right]$ here, since the estimates of $J_{8}\left[f_{n}\right]$ are obtained by the similar argument. By changing the order of the integration we see

$$
\int_{1}^{\infty}\left|J_{7}\left[f_{n}\right](r)\right| \mathrm{d} r \leq \int_{1}^{\infty} \int_{\tau}^{\infty} r\left|K_{\mu-1}(\sqrt{\lambda} r)\right| \mathrm{d} r\left|I_{\mu+1}(\sqrt{\lambda} \tau)\right|\left|f_{\theta, n}\right| \tau \mathrm{d} \tau .
$$

Then (340) and (341) combined with Lemmas A.2 and A.3 for the estimates of $I_{\mu+1}(\sqrt{\lambda} \tau)$ yield

$$
\begin{aligned}
& \text { R.H.S. of }(105) \leq \int_{1}^{\max \left\{\frac{1}{\Re(\sqrt{\lambda})}, 1\right\}} \int_{\tau}^{\infty}+\int_{\max \left\{\frac{1}{\Re(\sqrt{\lambda})}, 1\right\}}^{\infty} \int_{\tau}^{\infty} \\
& \leq C|\lambda|^{-\frac{\Re(\mu)+1}{2}} \int_{1}^{\max \left\{\frac{1}{\Re(\sqrt{\lambda})}, 1\right\}} \tau^{1-\Re(\mu)}|\sqrt{\lambda} \tau|^{\Re(\mu)+1}\left|f_{\theta, n}\right| \tau \mathrm{d} \tau \\
& \quad+C|\lambda|^{-1} \int_{1}^{\max \left\{\frac{1}{\Re(\sqrt{\lambda})}, 1\right\}}|\sqrt{\lambda} \tau|^{\Re(\mu)+1}\left|f_{\theta, n}\right| \tau \mathrm{d} \tau+C|\lambda|^{-1} \int_{1}^{\infty}\left|f_{\theta, n}\right| \tau \mathrm{d} \tau \\
& \leq C|\lambda|^{-1}\left\|f_{\theta, n}\right\|_{L^{1}(\Omega)} .
\end{aligned}
$$

Next we have from Lemma A.2, for $\Re(\sqrt{\lambda}) \leq r^{-1}$,

$$
\begin{aligned}
\left|J_{7}\left[f_{n}\right](r)\right| & \leq C r|\sqrt{\lambda} r|^{-\Re(\mu)+1} \int_{1}^{r}|\sqrt{\lambda} \tau|^{\Re(\mu)+1}\left|f_{\theta, n}\right| \tau \mathrm{d} \tau \\
& \leq C|\lambda| r^{-\Re(\mu)+2} \int_{1}^{r} \tau^{\Re(\mu)+2}\left|f_{\theta, n}\right| \mathrm{d} \tau
\end{aligned}
$$

which implies

$$
r^{-1}\left|J_{7}\left[f_{n}\right](r)\right| \leq \frac{C}{|\lambda|}\left\|f_{n}\right\|_{L^{\infty}(\Omega)}, \quad r^{-1}\left|J_{7}\left[f_{n}\right](r)\right| \leq C\left\|f_{n}\right\|_{L^{1}(\Omega)},
$$

in the case $\Re(\sqrt{\lambda}) \leq r^{-1}$. On the other hand, if $\Re(\sqrt{\lambda}) \geq r^{-1}$ then we have again from Lemma A.3,

$$
\begin{aligned}
\left|J_{7}\left[f_{n}\right](r)\right| \leq & C r|\sqrt{\lambda} r|^{-\frac{1}{2}} e^{-r \Re(\sqrt{\lambda})}\left(\left|\int_{1}^{\left.\max \frac{1}{\Re(\sqrt{\lambda})}, 1\right\}}\right|+\left|\int_{\max \left\{\frac{1}{\Re(\sqrt{\lambda})}, 1\right\}}^{r}\right|\right) \\
\leq & C|\lambda|^{-\frac{1}{4}} r^{\frac{1}{2}} e^{-r \Re(\sqrt{\lambda})}\left(\int_{1}^{\max \left\{\frac{1}{\Re(\sqrt{\lambda})}, 1\right\}}|\sqrt{\lambda} \tau|^{\Re(\mu)+1}\left|f_{\theta, n}\right| \tau \mathrm{d} \tau\right. \\
& \left.+\int_{\max \left\{\frac{1}{\Re(\sqrt{\lambda})}, 1\right\}}^{r}|\sqrt{\lambda} \tau|^{-\frac{1}{2}} e^{\tau \Re(\sqrt{\lambda})}\left|f_{\theta, n}\right| \tau \mathrm{d} \tau\right) \\
\leq & C|\lambda|^{-\frac{1}{4}} r^{\frac{1}{2}} e^{-r \Re(\sqrt{\lambda})} \int_{1}^{\left.\max \frac{1}{\Re(\sqrt{\lambda})}, 1\right\}}\left|f_{\theta, n}\right| \tau \mathrm{d} \tau \\
& +C|\lambda|^{-\frac{1}{2}} r^{\frac{1}{2}} e^{-r \Re(\sqrt{\lambda})} \int_{\max \left\{\frac{1}{\Re(\sqrt{\lambda})}, 1\right\}}^{r} e^{\tau \Re(\sqrt{\lambda})}\left|f_{\theta, n}\right| \tau^{\frac{1}{2}} \mathrm{~d} \tau .
\end{aligned}
$$


The estimate (108) leads to (107) also in the case $\Re(\sqrt{\lambda}) \geq r^{-1}$, for we have

$$
\int_{\frac{1}{\Re(\sqrt{\lambda})}}^{r} e^{\tau \Re(\sqrt{\lambda})} \tau^{\frac{1}{2}} \mathrm{~d} \tau \leq C|\lambda|^{-\frac{1}{2}} r^{\frac{1}{2}} e^{r \Re(\sqrt{\lambda})}, \quad \Re(\sqrt{\lambda}) \geq r^{-1},
$$

which should be used to derive the first estimate in (107). The second estimate in (107) is straightforward from (108), and we omit the details.

(3) Estimate of $J_{9}\left[f_{n}\right](1)$ : Let $\Re(\sqrt{\lambda}) \geq 1$. By using Lemma A.3 we have

$$
\left|\int_{1}^{\infty} K_{\mu-1}(\sqrt{\lambda} \tau) f_{\theta, n} \tau \mathrm{d} \tau\right| \leq C|\lambda|^{-\frac{1}{4}} \int_{1}^{\infty} \tau^{\frac{1}{2}} e^{-\tau \Re(\sqrt{\lambda})}\left|f_{\theta, n}\right| \mathrm{d} \tau,
$$

which implies from Lemma A.3 for $I_{\mu+1}(\sqrt{\lambda})$ that

$$
\left|J_{9}\left[f_{n}\right](1)\right| \leq C|\lambda|^{-\frac{1}{2}} \int_{1}^{\infty} \tau^{\frac{1}{2}} e^{-(\tau-1) \Re(\sqrt{\lambda})}\left|f_{\theta, n}\right| \mathrm{d} \tau .
$$

Hence, we obtain (91) and (92) in the case $\Re(\sqrt{\lambda}) \geq 1$. The proof is complete.

Next we estimate the other term in (80). We start from the counterpart of Lemma 3.6.

Lemma 3.9 Let $f_{n} \in \mathcal{P}_{n} C_{0}^{\infty}(\Omega)^{2}$. Then we have

$$
r^{|n|} \int_{r}^{\infty} s^{1-|n|} \Phi_{n, \lambda}\left[f_{n}\right](s) \mathrm{d} s=\sum_{l=10}^{17} J_{l}\left[f_{n}\right](r),
$$

where

$$
\begin{aligned}
& J_{10}\left[f_{n}\right](r)=-r^{|n|} \int_{r}^{\infty} \tau^{1-|n|} K_{\mu_{n}}(\sqrt{\lambda} \tau) \mathrm{d} \tau \int_{1}^{r} I_{\mu_{n}}(\sqrt{\lambda} \tau) g_{n} \mathrm{~d} \tau, \\
& J_{11}\left[f_{n}\right](r)=-r^{|n|} \int_{r}^{\infty} \int_{\tau}^{\infty} s^{1-|n|} K_{\mu_{n}}(\sqrt{\lambda} s) \mathrm{d} s I_{\mu_{n}}(\sqrt{\lambda} \tau) g_{n} \mathrm{~d} \tau, \\
& J_{12}\left[f_{n}\right](r)=-\left(\mu_{n}-|n|\right) r^{|n|} \int_{r}^{\infty} \tau^{-|n|} K_{\mu_{n}-1}(\sqrt{\lambda} \tau) \mathrm{d} \tau \int_{1}^{r} I_{\mu_{n}+1}(\sqrt{\lambda} \tau) f_{\theta, n} \tau \mathrm{d} \tau, \\
& J_{13}\left[f_{n}\right](r)=-\left(\mu_{n}-|n|\right) r^{|n|} \int_{r}^{\infty} \int_{\tau}^{\infty} s^{-|n|} K_{\mu_{n}-1}(\sqrt{\lambda} s) \mathrm{d} s I_{\mu_{n}+1}(\sqrt{\lambda} \tau) f_{\theta, n} \tau \mathrm{d} \tau, \\
& J_{14}\left[f_{n}\right](r)=r^{|n|} \int_{r}^{\infty} \int_{r}^{\tau} s^{1-|n|} I_{\mu_{n}}(\sqrt{\lambda} s) \mathrm{d} s K_{\mu_{n}}(\sqrt{\lambda} \tau) j_{n} \mathrm{~d} \tau \\
& J_{15}\left[f_{n}\right](r)=\left(\mu_{n}+|n|\right) r^{|n|} \int_{r}^{\infty} \int_{r}^{\tau} s^{-|n|} I_{\mu_{n}+1}(\sqrt{\lambda} s) \mathrm{d} s K_{\mu_{n}-1}(\sqrt{\lambda} \tau) f_{\theta, n} \tau \mathrm{d} \tau, \\
& J_{16}\left[f_{n}\right](r)=-r K_{\mu_{n}-1}(\sqrt{\lambda} r) \int_{1}^{r} I_{\mu_{n}+1}(\sqrt{\lambda} \tau) f_{\theta, n} \tau \mathrm{d} \tau, \\
& J_{17}\left[f_{n}\right](r)=-r I_{\mu_{n}+1}(\sqrt{\lambda} r) \int_{r}^{\infty} K_{\mu_{n}-1}(\sqrt{\lambda} \tau) f_{\theta, n} \tau \mathrm{d} \tau .
\end{aligned}
$$

Proof. Since the proof is similar to the one in Lemma 3.6 we only give a sketch of it here. 
From (58) we have

$$
\begin{aligned}
& r^{|n|} \int_{r}^{\infty} s^{1-|n|} \Phi_{n, \lambda}\left[f_{n}\right](s) \mathrm{d} s \\
& =-r^{|n|} \int_{r}^{\infty} s^{1-|n|} K_{\mu_{n}}(\sqrt{\lambda} s) \int_{1}^{s} I_{\mu_{n}}(\sqrt{\lambda} \tau) g_{n} \mathrm{~d} \tau \mathrm{d} s \\
& \quad-r^{|n|} \int_{r}^{\infty} s^{1-|n|} \sqrt{\lambda} K_{\mu_{n}}(\sqrt{\lambda} s) \int_{1}^{s} I_{\mu_{n}+1}(\sqrt{\lambda} \tau) f_{\theta, n} \tau \mathrm{d} \tau \mathrm{d} s \\
& \quad+r^{|n|} \int_{r}^{\infty} s^{1-|n|} I_{\mu_{n}}(\sqrt{\lambda} s) \int_{s}^{\infty} K_{\mu_{n}}(\sqrt{\lambda} \tau) j_{n} \mathrm{~d} \tau \mathrm{d} s \\
& \quad+r^{|n|} \int_{r}^{\infty} s^{1-|n|} \sqrt{\lambda} I_{\mu_{n}}(\sqrt{\lambda} s) \int_{s}^{\infty} K_{\mu_{n}-1}(\sqrt{\lambda} \tau) f_{\theta, n} \tau \mathrm{d} \tau \mathrm{d} s \\
& =I I_{1}+I I_{2}+I I_{3}+I I_{4} .
\end{aligned}
$$

As for $I I_{1}$, by decomposing the integral $\int_{1}^{s}$ into $\int_{1}^{r}+\int_{r}^{s}$ and by changing the order of the integration for the second term we obtain

$$
I I_{1}=J_{10}\left[f_{n}\right]+J_{11}\left[f_{n}\right] .
$$

Similarly we rewrite $I I_{2}$ as

$$
\begin{aligned}
I I_{2}= & -r^{|n|} \int_{r}^{\infty} \tau^{1-|n|} \sqrt{\lambda} K_{\mu_{n}}(\sqrt{\lambda} \tau) \mathrm{d} \tau \int_{1}^{r} I_{\mu_{n}+1}(\sqrt{\lambda} \tau) f_{\theta, n} \tau \mathrm{d} \tau \\
& -r^{|n|} \int_{r}^{\infty} \int_{\tau}^{\infty} s^{1-|n|} \sqrt{\lambda} K_{\mu_{n}}(\sqrt{\lambda} s) \mathrm{d} s I_{\mu_{n}+1}(\sqrt{\lambda} \tau) f_{\theta, n} \tau \mathrm{d} \tau
\end{aligned}
$$

As in the proof of Lemma 3.6, we recall the identity

$$
\sqrt{\lambda} K_{\mu_{n}}(\sqrt{\lambda} s)=\frac{\mu_{n}-1}{s} K_{\mu_{n}-1}(\sqrt{\lambda} s)-\frac{\mathrm{d}}{\mathrm{d} s} K_{\mu_{n}-1}(\sqrt{\lambda} s) .
$$

Then the integration by parts yields

$$
\begin{aligned}
I I_{2}= & J_{12}\left[f_{n}\right]+J_{13}\left[f_{n}\right]+J_{16}\left[f_{n}\right] \\
& -r^{|n|} \int_{r}^{\infty} \tau^{1-|n|} K_{\mu_{n}-1}(\sqrt{\lambda} \tau) I_{\mu_{n}+1}(\sqrt{\lambda} \tau) f_{\theta, n} \tau \mathrm{d} \tau .
\end{aligned}
$$

Next we see $I I_{3}=J_{14}\left[f_{n}\right]$ by changing the order of the integration. Similarly, we rewrite $I_{4}$ as

$$
I I_{4}=r^{|n|} \int_{r}^{\infty} \int_{r}^{\tau} s^{1-|n|} \sqrt{\lambda} I_{\mu_{n}}(\sqrt{\lambda} s) \mathrm{d} s K_{\mu_{n}-1}(\sqrt{\lambda} \tau) f_{\theta, n} \tau \mathrm{d} \tau .
$$

Then the identity

$$
\sqrt{\lambda} I_{\mu_{n}}(\sqrt{\lambda} s)=\frac{\mu_{n}+1}{s} I_{\mu_{n}+1}(\sqrt{\lambda} s)+\frac{\mathrm{d}}{\mathrm{d} s} I_{\mu_{n}+1}(\sqrt{\lambda} s) .
$$

and the integration by parts imply

$$
I I_{4}=J_{15}\left[f_{n}\right]+J_{17}\left[f_{n}\right]+r^{|n|} \int_{r}^{\infty} \tau^{1-|n|} I_{\mu_{n}+1}(\sqrt{\lambda} \tau) K_{\mu_{n}-1}(\sqrt{\lambda} \tau) f_{\theta, n} \tau \mathrm{d} \tau .
$$


Collecting these, we obtain (111). The proof is complete.

As in Lemma 3.7, we will estimate each $J_{l}$ under the condition $\Re(\mu)>|n|$ rather than the concrete value of $\mu_{n}(\alpha)$. Since the terms $J_{16}\left[f_{n}\right]$ and $J_{17}\left[f_{n}\right]$ are the same as $J_{7}\left[f_{n}\right]$ and $J_{8}\left[f_{n}\right]$, respectively, it suffices to estimate the other terms.

Lemma 3.10 Let $n \in \mathbb{Z} \backslash\{0\}, \Re(\mu)>|n|$, and let $\lambda \in \Sigma_{\pi-\epsilon}$ for some $\epsilon \in\left(0, \frac{\pi}{2}\right)$. Then there is a positive constant $C=C(\epsilon, n, \mu)$ such that the following statements hold.

(1) Let $l=10,12$, and let $f_{n} \in \mathcal{P}_{n} C_{0}^{\infty}(\Omega)^{2}$. Then

$$
\begin{aligned}
& \sup _{r \geq 1} r^{\frac{2}{q}-1}\left|J_{l}\left[f_{n}\right](r)\right| \leq \frac{C}{|\lambda|}\left\|f_{n}\right\|_{L^{q}(\Omega)}, \quad 1 \leq q \leq \infty . \\
& \sup _{r \geq 1} r^{-1}\left|J_{l}\left[f_{n}\right](r)\right| \leq C\left\|f_{n}\right\|_{L^{1}(\Omega)} .
\end{aligned}
$$

(2) Let $l=11,13,14,15$, and let $f_{n} \in \mathcal{P}_{n} C_{0}^{\infty}(\Omega)^{2}$. Then

$$
\begin{aligned}
& \sup _{r \geq 1} r^{\frac{2}{q}-1}\left|J_{l}\left[f_{n}\right](r)\right| \leq \frac{C_{q}}{|\lambda|}\left\|f_{n}\right\|_{L^{q}(\Omega)}, \quad 1 \leq q<\infty, \\
& \sup _{r \geq 1} r^{-1}\left|J_{l}\left[f_{n}\right](r)\right| \leq C\left\|f_{n}\right\|_{L^{1}(\Omega)} .
\end{aligned}
$$

Here the constant $C_{q}$ depends only on $\epsilon, n$, $\mu$, and $q$.

Remark 3.11 In fact, the estimate (117) is valid also for $q=\infty$ in the case $|n| \geq 2$. But this $L^{\infty}$ estimate does not hold if $|n|=1$ and $f_{n} \in C_{0}^{\infty}(\Omega)^{2}$. In order to establish the $L^{\infty}$ estimate for $|n|=1$ we need to use the additional condition $\operatorname{div} f_{n}=0$, which will be shown later in Lemmas 3.13, 3.14, and 3.15.

Proof of Lemma 3.10. (1) Here we give a proof of the estimates only for $J_{10}\left[f_{n}\right]$. The estimates of $J_{12}\left[f_{n}\right]$ are obtained in the similar manner. Let $\Re(\sqrt{\lambda}) \leq r^{-1}$. Then, applying (335) and Lemma A.2, we have

$$
\begin{aligned}
\left|J_{10}\left[f_{n}\right](r)\right| & \leq C r^{|n|}|\lambda|^{-\frac{\Re(\mu)}{2}} r^{2-|n|-\Re(\mu)} \int_{1}^{r}|\sqrt{\lambda} \tau|^{\Re(\mu)}\left|g_{n}\right| \mathrm{d} \tau \\
& \leq C r^{2-\Re(\mu)} \int_{1}^{r}\left|g_{n}\right| \tau^{\Re(\mu)} \mathrm{d} \tau
\end{aligned}
$$

This estimate implies, for $\Re(\sqrt{\lambda}) \leq r^{-1}$,

$$
\begin{aligned}
r^{\frac{2}{q}-1}\left|J_{10}\left[f_{n}\right](r)\right| & \leq \frac{C}{|\lambda|}\left\|f_{n}\right\|_{L^{q}(\Omega)}, \quad 1 \leq q \leq \infty, \\
r^{-1}\left|J_{10}\left[f_{n}\right](r)\right| & \leq C\left\|f_{n}\right\|_{L^{1}(\Omega)} .
\end{aligned}
$$

Note that (120) and (121) are proved even in the case $\Re(\sqrt{\lambda}) \leq 2 r^{-1}$ with a trivial modification. Thus we next consider the case $\Re(\sqrt{\lambda}) \geq 2 r^{-1}$. In this case we have from (334), 
Lemma A.2, and Lemma A.3,

$$
\begin{aligned}
\left|J_{10}\left[f_{n}\right](r)\right| \leq & C r^{|n|}|\lambda|^{-\frac{3}{4}} r^{\frac{1}{2}-|n|} e^{-r \Re(\sqrt{\lambda})}\left(\int_{1}^{\max \left\{\frac{2}{\Re(\sqrt{\lambda})}, 1\right\}}\left|I_{\mu}(\sqrt{\lambda} \tau)\right|\left|g_{n}\right| \mathrm{d} \tau\right. \\
& \left.+\int_{\max \left\{\frac{2}{\Re(\sqrt{\lambda})}, 1\right\}}^{r}\left|I_{\mu}(\sqrt{\lambda} \tau)\right|\left|g_{n}\right| \mathrm{d} \tau\right) \\
\leq & C|\lambda|^{-\frac{3}{4}} r^{\frac{1}{2}} e^{-r \Re(\sqrt{\lambda})}\left(|\lambda|^{\Re(\mu)} \frac{{ }^{2}}{\max \left\{\frac{2}{\Re(\sqrt{\lambda})}, 1\right\}}\left|g_{n}\right| \tau^{\Re(\mu)} \mathrm{d} \tau\right. \\
& \left.+|\lambda|^{-\frac{1}{4}} \int_{\max \left\{\frac{2}{\Re(\sqrt{\lambda})}, 1\right\}}^{r} \tau^{-\frac{1}{2}} e^{\tau \Re(\sqrt{\lambda})}\left|g_{n}\right| \mathrm{d} \tau\right) .
\end{aligned}
$$

Hence, it is easy to see from (122) that (120) and (121) hold also in the case $\Re(\sqrt{\lambda}) \geq 2 r^{-1}$, by virtue of the estimates for $\Re(\sqrt{\lambda}) \geq 2 r^{-1}$ and $\Re(\mu)>|n| \geq 1$ :

$$
\begin{aligned}
& \int_{1}^{\max \left\{\frac{2}{\Re(\sqrt{\lambda})}, 1\right\}}\left|g_{n}\right| \tau^{\Re(\mu)} \mathrm{d} \tau \leq C|\lambda|^{-\frac{\Re(\mu)-1}{2}} \int_{1}^{\max \left\{\frac{2}{\Re(\sqrt{\lambda})}, 1\right\}}\left|g_{n}\right| \tau \mathrm{d} \tau \\
& \int_{\max \left\{\frac{2}{\Re(\sqrt{\lambda})}, 1\right\}}^{r} \tau^{-\frac{3}{2}} e^{\tau \Re(\sqrt{\lambda})}\left|g_{n}\right| \tau \mathrm{d} \tau \leq C r^{-\frac{3}{2}} e^{r \Re(\sqrt{\lambda})}\left\|g_{n}\right\|_{L^{1}(\Omega)} \\
& \int_{\max \left\{\frac{2}{\Re(\sqrt{\lambda})}, 1\right\}}^{r} \tau^{-\frac{1}{2}} e^{\tau \Re(\sqrt{\lambda})}\left|g_{n}\right| \mathrm{d} \tau \\
& \leq C|\lambda|^{-\frac{1}{2}}\left(r^{-\frac{1}{2}} e^{r \Re(\sqrt{\lambda})}+\frac{1}{2} \int_{\max \left\{\frac{2}{\Re(\sqrt{\lambda})}, 1\right\}}^{r} \tau^{-\frac{3}{2}} e^{\tau \Re(\sqrt{\lambda})}\right)\left\|g_{n}\right\|_{L^{\infty}(\Omega)} \\
& \leq C|\lambda|^{-\frac{1}{2}} r^{-\frac{1}{2}} e^{r \Re(\sqrt{\lambda})}\left\|g_{n}\right\|_{L^{\infty}(\Omega)} .
\end{aligned}
$$

Note that the last two estimates are verified by using the fact that $s^{-\frac{3}{2}} e^{s}$ is monotone increasing for $s \geq 2$. The proof of (1) is complete.

(2) (i) Estimate of $J_{11}\left[f_{n}\right]$ and $J_{13}\left[f_{n}\right]$ : We will give a proof only for $J_{11}\left[f_{n}\right]$, since the proof of $J_{13}\left[f_{n}\right]$ is similar. Let $\Re(\sqrt{\lambda}) \geq r^{-1}$. Then (334) and Lemma A.3 imply

$$
\begin{aligned}
\left|J_{11}\left[f_{n}\right](r)\right| & \leq C|\lambda|^{-\frac{3}{4}} r|n| \int_{r}^{\infty} \tau^{\frac{1}{2}-|n|} e^{-\tau \Re(\sqrt{\lambda})}|\sqrt{\lambda} \tau|^{-\frac{1}{2}} e^{\tau \Re(\sqrt{\lambda})}\left|g_{n}\right| \mathrm{d} \tau \\
& \leq C|\lambda|^{-1} r^{|n|} \int_{r}^{\infty} \tau^{-|n|}\left|g_{n}\right| \mathrm{d} \tau .
\end{aligned}
$$

Hence we have for $\Re(\sqrt{\lambda}) \geq r^{-1}$ and $l=11$,

$$
\begin{aligned}
r^{\frac{2}{q}-1}\left|J_{l}\left[f_{n}\right](r)\right| & \leq \frac{C}{|\lambda|}\left\|f_{n}\right\|_{L^{q}(\Omega)}, \quad 1 \leq q<\infty, \\
r^{-1}\left|J_{l}\left[f_{n}\right](r)\right| & \leq C\left\|f_{n}\right\|_{L^{1}(\Omega)} .
\end{aligned}
$$

Next we consider the case $\Re(\sqrt{\lambda}) \leq r^{-1}$. Then we divide the integral into $-r^{|n|} \int_{r}^{\frac{1}{\Re(\sqrt{\lambda})}} \int_{\tau}^{\infty}$ and $-r^{|n|} \int_{\frac{1}{\Re(\sqrt{\lambda})}}^{\infty} \int_{\tau}^{\infty}$ and the second term is bounded from above by

$$
C|\lambda|^{-1} r^{|n|} \int_{\frac{1}{\Re(\sqrt{\lambda})}}^{\infty} \tau^{-|n|}\left|g_{n}\right| \mathrm{d} \tau
$$


as in (123). On the other hand, we have from (335) and Lemma A.2,

$$
\begin{aligned}
r^{|n|}\left|\int_{r} \frac{1}{\Re(\sqrt{\lambda})} \int_{\tau}^{\infty}\right| & \leq C r^{|n|} \int_{r}^{\frac{1}{\Re(\sqrt{\lambda})}}|\lambda|^{-\frac{\Re(\mu)}{2}} \tau^{2-|n|-\Re(\mu)}|\sqrt{\lambda} \tau|^{\Re(\mu)}\left|g_{n}\right| \mathrm{d} \tau \\
& \leq C r^{|n|} \int_{r}^{\frac{1}{\Re(\sqrt{\lambda})}} \tau^{2-|n|}\left|g_{n}\right| \mathrm{d} \tau
\end{aligned}
$$

Collecting these, we obtain (124) and (125) also for the case $\Re(\sqrt{\lambda}) \leq r^{-1}$ and $l=11$. For example, (124) is obtained by using $\int_{r}^{\frac{1}{\Re(\sqrt{\lambda})}} \tau^{2-|n|}\left|g_{n}\right| \mathrm{d} \tau \leq C|\lambda|^{-1} \int_{r}^{\frac{1}{\Re(\sqrt{\lambda})}} \tau^{-|n|}\left|g_{n}\right| \mathrm{d} \tau$ and then applying the Hölder inequality. The details are omitted here.

(ii) Estimate of $J_{14}\left[f_{n}\right]$ and $J_{15}\left[f_{n}\right]$ : We will give a proof only for $J_{14}\left[f_{n}\right]$, since the proof of $J_{15}\left[f_{n}\right]$ is similar. Let $\Re(\sqrt{\lambda}) \geq r^{-1}$. Then (339) and Lemma A.3 lead to

$$
\begin{aligned}
& \left|J_{14}\left[f_{n}\right](r)\right| \\
& \leq C r^{|n|} \int_{r}^{\infty}\left(|\lambda|^{\mid \frac{|n|}{2}-1}+|\lambda|^{-\frac{3}{4}} \tau^{\frac{1}{2}-|n|} e^{\tau \Re(\sqrt{\lambda})}\right)|\sqrt{\lambda} \tau|^{-\frac{1}{2}} e^{-\tau \Re(\sqrt{\lambda})}\left|j_{n}\right| \mathrm{d} \tau \\
& \leq C|\lambda|^{\frac{|n|}{2}-\frac{5}{4}} r^{|n|} \int_{r}^{\infty} \tau^{-\frac{1}{2}} e^{-\tau \Re(\sqrt{\lambda})}\left|j_{n}\right| \mathrm{d} \tau \\
& \quad+C|\lambda|^{-1} r^{|n|} \int_{r}^{\infty} \tau^{-|n|}\left|j_{n}\right| \mathrm{d} \tau,
\end{aligned}
$$

which implies (124) and (125) for $\Re(\sqrt{\lambda}) \geq r^{-1}$ and $l=14$. Next we consider the case $\Re(\sqrt{\lambda}) \leq r^{-1}$. Then we have from (338), (339), and Lemmas A.2, A.3,

$$
\begin{aligned}
& \left|J_{14}\left[f_{n}\right](r)\right| \\
& \leq r^{|n|}\left|\int_{r}^{\frac{1}{\Re(\sqrt{\lambda})}} \int_{r}^{\tau}\right|+r^{|n|}\left|\int_{\frac{1}{\Re(\sqrt{\lambda})}}^{\infty} \int_{r}^{\tau}\right| \\
& \leq C r^{|n|} \int_{r}^{\frac{1}{\Re(\sqrt{\lambda})}}|\lambda|^{\frac{\Re(\mu)}{2}} \tau^{\Re(\mu)+2-|n|}|\sqrt{\lambda} \tau|^{-\Re(\mu)}\left|j_{n}\right| \mathrm{d} \tau \\
& \quad+C r^{|n|} \int_{\frac{1}{\Re(\sqrt{\lambda})}}^{\infty}\left(|\lambda|^{\frac{|n|}{2}-1}+|\lambda|^{-\frac{3}{4}} \tau^{\frac{1}{2}-|n|} e^{\tau \Re(\sqrt{\lambda})}\right)|\sqrt{\lambda} \tau|^{-\frac{1}{2}} e^{-\tau \Re(\sqrt{\lambda})}\left|j_{n}\right| \mathrm{d} \tau \\
& \leq C r^{|n|} \int_{r}^{\frac{1}{\Re(\sqrt{\lambda})}} \tau^{2-|n|}\left|j_{n}\right| \mathrm{d} \tau \\
& \quad+C|\lambda|^{|n|}-\frac{5}{4} r^{|n|} \int_{\frac{1}{\Re(\sqrt{\lambda})}}^{\infty} \tau^{-\frac{1}{2}} e^{-\tau \Re(\sqrt{\lambda})}\left|j_{n}\right| \mathrm{d} \tau+C|\lambda|^{-1} r^{|n|} \int_{\frac{1}{\Re(\sqrt{\lambda})}}^{\infty} \tau^{-|n|}\left|j_{n}\right| \mathrm{d} \tau .
\end{aligned}
$$

Thus it is easy to see from (128) that (124) and (125) hold for $\Re(\sqrt{\lambda}) \leq r^{-1}$ and $l=14$. The proof of (2) is complete.

Lemma 3.7 and Lemma 3.10 imply the $L^{p}-L^{q}$ estimates of the operator $J_{l}[\cdot]$. In the next corollary we always assume the conditions $\lambda \in \Sigma_{\pi-\epsilon}$ for some $\epsilon \in\left(0, \frac{\pi}{2}\right)$ and $\Re(\mu)>|n|$, and the constant $C$ appearing in the estimates depends only on $\epsilon, n, \mu, p$, and $q$, although these are abbreviated in the statements. We will also identify the function $g=g(r)$ for $r \in(1, \infty)$ with the radially symmetric function $g(|x|)$ in $\Omega$, and we denote by $\|g\|_{L^{p}(\Omega)}$ the $L^{p}$ norm of $g(|x|)$ in $\Omega$. 
Corollary 3.12 (i) Let $l \in\{1, \cdots, 8\} \cup\{10,12,16,17\}$ and let $1 \leq q<p \leq \infty$ or $1<q \leq p \leq \infty$. Then each $r^{-1} J_{l}[\cdot]$ is extended to a bounded linear operator from $L^{q}(\Omega)^{2}$ to $L^{p}(\Omega)$ and satisfies

$$
\left\|r^{-1} J_{l}\left[f_{n}\right]\right\|_{L^{p}(\Omega)} \leq \frac{C}{|\lambda|^{1+\frac{1}{p}-\frac{1}{q}}}\left\|f_{n}\right\|_{L^{q}(\Omega)} .
$$

(ii) Let $l \in\{11,13,14,15\}$ and let $1 \leq q<p \leq \infty$ or $1<q \leq p<\infty$. Then each $r^{-1} J_{l}[\cdot]$ is extended to a bounded linear operator from $L^{q}(\Omega)^{2}$ to $L^{p}(\Omega)$ and satisfies (129).

(iii) Let $l \in\{11,13,14,15\}$ and let $1 \leq q<\infty$. Then each $J_{l}[\cdot](1)$ is extended to a bounded linear operator from $L^{q}(\Omega)^{2}$ to $\mathbb{C}$ and satisfies

$$
\left|J_{l}\left[f_{n}\right](1)\right| \leq \frac{C}{|\lambda|^{1-\frac{1}{q}}\left(1+|\lambda|^{\frac{1}{q}}\right)}\left\|f_{n}\right\|_{L^{q}(\Omega)} .
$$

Proof. (i) The estimates (86) and (115) in Lemmas 3.7, 3.10 imply that each $r^{-1} J_{l}[\cdot]$, $l \in\{1, \cdots, 8\} \cup\{10,12,16,17\}$, is extended to a bounded linear operator from $L^{1}(\Omega)^{2}$ to weak- $L^{1}(\Omega)$ and from $L^{\infty}(\Omega)^{2}$ to $L^{\infty}(\Omega)$ with the estimate $C|\lambda|^{-1}$ of its operator norm. Hence, by the Marcinkiewicz interpolation theorem each $r^{-1} J_{l}[\cdot]$ is extended to a bounded linear operator from $L^{q}(\Omega)^{2}$ to $L^{q}(\Omega)$ for all $1<q \leq \infty$, and the estimate (129) holds for $p=q$. Moreover, the estimates (87) and (116) combined with the above result for the case $q=\infty$ yields that, again by the interpolation theorem, each $r^{-1} J_{l}[\cdot]$ is bounded from $L^{q}(\Omega)^{2}$ to $L^{\infty}(\Omega)$ with the estimate $C|\lambda|^{1-\frac{1}{q}}$ of its operator norm. Then again from the Marcinkiewicz interpolation theorem each $r^{-1} J_{l}[\cdot]$ is extended to a bounded linear operator from $L^{q}(\Omega)^{2}$ to $L^{p}(\Omega)$ for all $1<q \leq p \leq \infty$, together with the estimate (129).

(ii) The estimate (117) implies that each $r^{-1} J_{l}[\cdot]$ with $l \in\{11,13,14,15\}$, is extended to a bounded linear operator from $L^{q}(\Omega)^{2}$ to weak- $L^{q}(\Omega)$ for all $1 \leq q<\infty$ with the estimate $C|\lambda|^{-1}$ of its operator norm. Thus by the Marcinkiewicz interpolation theorem each $r^{-1} J_{l}[\cdot]$ is bounded from $L^{q}(\Omega)^{2}$ to $L^{q}(\Omega)$ for each $1<q<\infty$ with the estimate $C|\lambda|^{-1}$ of its operator norm. On the other hand, we observe from (117) with $q=1$ and (118) that $r^{-1} J_{l}[\cdot]$ is bounded from $L^{1}(\Omega)^{2}$ to $L^{p}(\Omega)$ for $1<p \leq \infty$ with the estimate $C|\lambda|^{-\frac{1}{p}}$ of its operator norm. Hence, again from the interpolation theorem each $r^{-1} J_{l}[\cdot]$ is bounded from $L^{q}(\Omega)^{2}$ to $L^{p}(\Omega)$ for any $1<q \leq p<\infty$, with the estimate as in (129).

(iii) If $\Re(\sqrt{\lambda}) \leq 1$ then (130) follows from (ii) with $p=\infty$. If $\Re(\sqrt{\lambda}) \geq 1$ then (130) follows from (117). The proof is complete.

In Corollary 3.12 the $L^{\infty}-L^{\infty}$ estimate of $J_{l}[\cdot]$ is lacking for $l=11,13,14,15$. For completeness we will derive the estimate of $J_{l}[\cdot]$ in $L^{\infty}(\Omega)$ for $f_{n} \in \mathcal{P}_{n} C_{0, \sigma}^{\infty}(\Omega)$ below. As will be seen in the next lemma, the divergence free condition on $f_{n}$ plays an important role to obtain the estimate in $L^{\infty}(\Omega)$, while such a condition is not necessary in Corollary 3.12. For simplicity of notations we will write $\mu$ for $\mu_{n}=\mu_{n}(\alpha)$.

Lemma 3.13 Set

$$
L_{\mu}(z)=K_{\mu-1}(z) I_{\mu}(z)+K_{\mu}(z) I_{\mu+1}(z) .
$$

Then for any $f_{n} \in \mathcal{P}_{n} C_{0, \sigma}^{\infty}(\Omega)$ we have

$$
J_{11}\left[f_{n}\right]+J_{13}\left[f_{n}\right]+J_{14}\left[f_{n}\right]+J_{15}\left[f_{n}\right]=\sum_{l=18}^{26} J_{l}\left[f_{n}\right],
$$


where

$$
\begin{aligned}
& J_{18}\left[f_{n}\right](r)=\frac{(|n|-\mu) r^{|n|}}{\sqrt{\lambda}} \int_{r}^{\infty} \int_{\tau}^{\infty} s^{-|n|} K_{\mu-1}(\sqrt{\lambda} s) \mathrm{d} s I_{\mu}(\sqrt{\lambda} \tau) g_{n} \mathrm{~d} \tau, \\
& J_{19}\left[f_{n}\right](r)=\frac{\left(\mu^{2}-|n|^{2}\right) r^{|n|}}{\sqrt{\lambda}} \int_{r}^{\infty} \int_{\tau}^{\infty} s^{-1-|n|} K_{\mu}(\sqrt{\lambda} s) \mathrm{d} s I_{\mu+1}(\sqrt{\lambda} \tau) f_{\theta, n} \tau \mathrm{d} \tau, \\
& J_{20}\left[f_{n}\right](r)=\frac{(|n|+\mu) r^{|n|}}{\sqrt{\lambda}} \int_{r}^{\infty} \int_{r}^{\tau} s^{-|n|} I_{\mu+1}(\sqrt{\lambda} s) \mathrm{d} s K_{\mu}(\sqrt{\lambda} \tau) j_{n} \mathrm{~d} \tau \\
& J_{21}\left[f_{n}\right](r)=\frac{\left(|n|^{2}-\mu^{2}\right) r^{|n|}}{\sqrt{\lambda}} \int_{r}^{\infty} \int_{r}^{\tau} s^{-1-|n|} I_{\mu}(\sqrt{\lambda} s) \mathrm{d} s K_{\mu-1}(\sqrt{\lambda} \tau) f_{\theta, n} \mathrm{~d} \tau, \\
& J_{22}\left[f_{n}\right](r)=-\frac{r}{\sqrt{\lambda}} I_{\mu+1}(\sqrt{\lambda} r) \int_{r}^{\infty} K_{\mu}(\sqrt{\lambda} \tau) j_{n} \mathrm{~d} \tau \\
& J_{23}\left[f_{n}\right](r)=-\frac{\mu+|n|}{\sqrt{\lambda}} I_{\mu}(\sqrt{\lambda} r) \int_{r}^{\infty} K_{\mu-1}(\sqrt{\lambda} \tau) f_{\theta, n} \tau \mathrm{d} \tau \\
& J_{24}\left[f_{n}\right](r)=\frac{i n(1-|n|) r^{|n|}}{|n| \sqrt{\lambda}} \int_{r}^{\infty} \tau^{1-|n|} L_{\mu}(\sqrt{\lambda} \tau) f_{r, n} \mathrm{~d} \tau \\
& J_{25}\left[f_{n}\right](r)=-\frac{i n r^{2}}{|n| \sqrt{\lambda}} L_{\mu}(\sqrt{\lambda} r) f_{r, n}(r) \\
& J_{26}\left[f_{n}\right](r)=-\frac{i n r^{|n|}}{|n| \sqrt{\lambda}} \int_{r}^{\infty} \frac{\mathrm{d}}{\mathrm{d} \tau}\left(\tau^{2-|n|} L_{\mu}(\sqrt{\lambda} \tau)\right) f_{r, n} \mathrm{~d} \tau .
\end{aligned}
$$

Proof. By using $K_{\mu}(z)=\frac{\mu-1}{z} K_{\mu-1}(z)-\frac{\mathrm{d} K_{\mu-1}}{\mathrm{~d} z}(z)$ from (327) and by the integration by parts, we have

$$
\begin{aligned}
J_{11}\left[f_{n}\right](r)= & \frac{(|n|-\mu) r^{|n|}}{\sqrt{\lambda}} \int_{r}^{\infty} \int_{\tau}^{\infty} s^{-|n|} K_{\mu-1}(\sqrt{\lambda} s) \mathrm{d} s I_{\mu}(\sqrt{\lambda} \tau) g_{n} \mathrm{~d} \tau \\
& -\frac{r^{|n|}}{\sqrt{\lambda}} \int_{r}^{\infty} \tau^{1-|n|} K_{\mu-1}(\sqrt{\lambda} \tau) I_{\mu}(\sqrt{\lambda} \tau) g_{n} \mathrm{~d} \tau .
\end{aligned}
$$

Similarly, from $K_{\mu-1}(z)=-\frac{\mu}{z} K_{\mu}(z)-\frac{\mathrm{d} K_{\mu}}{\mathrm{d} z}(z)$ and by the integration by parts, we see

$$
\begin{aligned}
J_{13}\left[f_{n}\right](r)= & \frac{\left(\mu^{2}-|n|^{2}\right) r^{|n|}}{\sqrt{\lambda}} \int_{r}^{\infty} \int_{\tau}^{\infty} s^{-1-|n|} K_{\mu}(\sqrt{\lambda} s) \mathrm{d} s I_{\mu+1}(\sqrt{\lambda} \tau) f_{\theta, n} \tau \mathrm{d} \tau \\
& +\frac{(|n|-\mu) r^{|n|}}{\sqrt{\lambda}} \int_{r}^{\infty} \tau^{1-|n|} K_{\mu}(\sqrt{\lambda} \tau) I_{\mu+1}(\sqrt{\lambda} \tau) f_{\theta, n} \mathrm{~d} \tau .
\end{aligned}
$$

Next, the identity $I_{\mu}(z)=\frac{\mu+1}{z} I_{\mu+1}(z)+\frac{\mathrm{d} I_{\mu+1}}{\mathrm{~d} z}(z)$ from (324) and the integration by parts yield

$$
\begin{aligned}
J_{14}\left[f_{n}\right](r)= & \frac{(|n|+\mu) r^{|n|}}{\sqrt{\lambda}} \int_{r}^{\infty} \int_{r}^{\tau} s^{-|n|} I_{\mu+1}(\sqrt{\lambda} s) \mathrm{d} s K_{\mu}(\sqrt{\lambda} \tau) j_{n} \mathrm{~d} \tau \\
& -\frac{r}{\sqrt{\lambda}} I_{\mu+1}(\sqrt{\lambda} r) \int_{r}^{\infty} K_{\mu}(\sqrt{\lambda} \tau) j_{n} \mathrm{~d} \tau \\
& +\frac{r^{|n|}}{\sqrt{\lambda}} \int_{r}^{\infty} \tau^{1-|n|} K_{\mu}(\sqrt{\lambda} \tau) I_{\mu+1}(\sqrt{\lambda} \tau) j_{n} \mathrm{~d} \tau
\end{aligned}
$$


Similarly, we have from $I_{\mu+1}(z)=-\frac{\mu}{z} I_{\mu}(z)+\frac{\mathrm{d} I_{\mu}}{\mathrm{d} z}(z)$ due to (324) and from the integration by parts,

$$
\begin{aligned}
J_{15}\left[f_{n}\right](r)= & \frac{\left(|n|^{2}-\mu^{2}\right) r^{|n|}}{\sqrt{\lambda}} \int_{r}^{\infty} \int_{r}^{\tau} s^{-1-|n|} I_{\mu}(\sqrt{\lambda} s) \mathrm{d} s K_{\mu-1}(\sqrt{\lambda} \tau) f_{\theta, n} \mathrm{~d} \tau \\
& -\frac{\mu+|n|}{\sqrt{\lambda}} I_{\mu}(\sqrt{\lambda} r) \int_{r}^{\infty} K_{\mu-1}(\sqrt{\lambda} \tau) f_{\theta, n} \tau \mathrm{d} \tau \\
& +\frac{(\mu+|n|) r|n|}{\sqrt{\lambda}} \int_{r}^{\infty} \tau^{1-|n|} K_{\mu-1}(\sqrt{\lambda} \tau) I_{\mu}(\sqrt{\lambda} \tau) f_{\theta, n} \mathrm{~d} \tau
\end{aligned}
$$

Since $g_{n}=\mu f_{\theta, n}+i n f_{r, n}$ and $j_{n}=\mu f_{\theta, n}-i n f_{r, n}$, the sum of the last terms in each of (133) - (136) is given as

$$
\frac{r^{|n|}}{\sqrt{\lambda}} \int_{r}^{\infty} \tau^{1-|n|} L_{\mu}(\sqrt{\lambda} \tau)\left(|n| f_{\theta, n}-i n f_{r, n}\right) \mathrm{d} \tau
$$

where $L_{\mu}(z)$ is given as (131). Then the term (137) is equal to, by the divergence free condition $i n f_{\theta, n}=-f_{r, n}-r \frac{\mathrm{d} f_{r, n}}{\mathrm{~d} r}$,

$$
\begin{aligned}
& \frac{i n(1-|n|) r^{|n|}}{|n| \sqrt{\lambda}} \int_{r}^{\infty} \tau^{1-|n|} L_{\mu}(\sqrt{\lambda} \tau) f_{r, n} \mathrm{~d} \tau-\frac{i n r^{2}}{|n| \sqrt{\lambda}} L_{\mu}(\sqrt{\lambda} r) f_{r, n}(r) \\
& -\frac{i n r^{|n|}}{|n| \sqrt{\lambda}} \int_{r}^{\infty} \frac{\mathrm{d}}{\mathrm{d} \tau}\left(\tau^{2-|n|} L_{\mu}(\sqrt{\lambda} \tau)\right) f_{r, n} \mathrm{~d} \tau .
\end{aligned}
$$

Collecting these facts, we obtain (132). The proof is complete.

In the following lemmas we focus on the $L^{\infty}-L^{\infty}$ estimate, although it is possible to obtain the $L^{\infty}-L^{1}$ estimate as in the previous lemmas of this section. In Lemmas 3.14 and 3.15 below we always assume that $n \in \mathbb{Z} \backslash\{0\}, \Re(\mu)>|n|$, and $\lambda \in \Sigma_{\pi-\epsilon}$ for some $\epsilon \in\left(0, \frac{\pi}{2}\right)$. The constant $C$ in the estimates depends only on $\epsilon, n$, and $\mu$.

Lemma 3.14 Let $l=18, \cdots, 23$, and let $f_{n} \in \mathcal{P}_{n} C_{0}^{\infty}(\Omega)^{2}$. Then

$$
\sup _{r \geq 1} r^{-1}\left|J_{l}\left[f_{n}\right](r)\right| \leq \frac{C}{|\lambda|}\left\|f_{n}\right\|_{L^{\infty}(\Omega)} .
$$

Proof. (i) Estimate of $J_{18}\left[f_{n}\right]$ : Let $\Re(\sqrt{\lambda}) \geq r^{-1}$. Then (334) and Lemma A.3 imply

$$
\begin{aligned}
\left|J_{18}\left[f_{n}\right](r)\right| & \leq C|\lambda|^{-\frac{1}{2}} r^{|n|} \int_{r}^{\infty}|\lambda|^{-\frac{3}{4}} \tau^{-\frac{1}{2}-|n|} e^{-\tau \Re(\sqrt{\lambda})}|\sqrt{\lambda} \tau|^{-\frac{1}{2}} e^{\tau \Re(\sqrt{\lambda})}\left|g_{n}\right| \mathrm{d} \tau \\
& =C|\lambda|^{-\frac{3}{2}} r^{|n|} \int_{r}^{\infty} \tau^{-1-|n|}\left|g_{n}\right| \mathrm{d} \tau
\end{aligned}
$$

which yields for $\Re(\sqrt{\lambda}) \geq r^{-1}$ and $l=18$,

$$
r^{-1}\left|J_{l}\left[f_{n}\right](r)\right| \leq \frac{C}{|\lambda|}\left\|f_{n}\right\|_{L^{\infty}(\Omega)} .
$$


On the other hand, if $\Re(\sqrt{\lambda}) \leq r^{-1}$ then (334) and (335) together with Lemmas A.2, A.3 give

$$
\begin{aligned}
\left|J_{18}\left[f_{n}\right](r)\right| \leq & C|\lambda|^{-\frac{1}{2}} r^{|n|}\left(\left|\int_{r}^{\frac{1}{\Re(\sqrt{\lambda})}} \int_{\tau}^{\infty}\right|+\left|\int_{\frac{1}{\Re(\sqrt{\lambda})}}^{\infty} \int_{\tau}^{\infty}\right|\right) \\
\leq & C|\lambda|^{-\frac{1}{2}} r^{|n|}\left(\int_{r}^{\frac{1}{\Re(\sqrt{\lambda})}}|\lambda|^{-\frac{\Re(\mu)-1}{2}} \tau^{2-|n|-\Re(\mu)}|\sqrt{\lambda} \tau|^{\Re(\mu)}\left|g_{n}\right| \mathrm{d} \tau\right. \\
& \left.+\int_{\frac{1}{\Re(\sqrt{\lambda})}}^{\infty}|\lambda|^{-\frac{3}{4}} \tau^{-\frac{1}{2}-|n|} e^{-\tau \Re(\sqrt{\lambda})}|\sqrt{\lambda} \tau|^{-\frac{1}{2}} e^{\tau \Re(\sqrt{\lambda})}\left|g_{n}\right| \mathrm{d} \tau\right) \\
\leq & C r^{|n|} \int_{r}^{\frac{1}{\Re(\sqrt{\lambda})}} \tau^{2-|n|}\left|g_{n}\right| \mathrm{d} \tau+C|\lambda|^{-\frac{3}{2}} r^{|n|} \int_{\frac{1}{\Re(\sqrt{\lambda})}}^{\infty} \tau^{-1-|n|}\left|g_{n}\right| \mathrm{d} \tau .
\end{aligned}
$$

Then it is easy to check (141) for $\Re(\sqrt{\lambda}) \leq r^{-1}$ and $l=18$.

(ii) Estimate of $J_{19}\left[f_{n}\right]$ : By the inequality

$$
\int_{\tau}^{\infty} s^{-1-|n|}\left|K_{\mu}(\sqrt{\lambda} s)\right| \mathrm{d} s \leq \tau^{-1} \int_{\tau}^{\infty} s^{-|n|}\left|K_{\mu}(\sqrt{\lambda} s)\right| \mathrm{d} s,
$$

the proof is a minor modification of the proof of the estimate for $J_{18}\left[f_{n}\right]$ above. Thus we omit the details.

(iii) Estimate of $J_{20}\left[f_{n}\right]$ : Let $\Re(\sqrt{\lambda}) \geq r^{-1}$. Then (339) and Lemma A.3 imply

$$
\begin{aligned}
& \left|J_{20}\left[f_{n}\right](r)\right| \\
& \leq C|\lambda|^{-\frac{1}{2}} r^{|n|} \int_{r}^{\infty}\left(|\lambda|^{\frac{|n|-1}{2}}+|\lambda|^{-\frac{3}{4}} \tau^{-\frac{1}{2}-|n|} e^{\tau \Re(\sqrt{\lambda})}\right)|\sqrt{\lambda} \tau|^{-\frac{1}{2}} e^{-\tau \Re(\sqrt{\lambda})}\left|j_{n}\right| \mathrm{d} \tau \\
& \leq C|\lambda|^{\frac{|n|}{2}-\frac{5}{4}} r^{|n|} \int_{r}^{\infty} \tau^{-\frac{1}{2}} e^{-\tau \Re(\sqrt{\lambda})}\left|j_{n}\right| \mathrm{d} \tau+C|\lambda|^{-\frac{3}{2}} r^{|n|} \int_{r}^{\infty} \tau^{-1-|n|}\left|j_{n}\right| \mathrm{d} \tau
\end{aligned}
$$

which yields the estimate (141) for $\Re(\sqrt{\lambda}) \geq r^{-1}$ and $l=20$. In the case $\Re(\sqrt{\lambda}) \leq r^{-1}$ we divide the integral into $\int_{r}^{\frac{1}{\Re(\sqrt{\lambda})}} \int_{r}^{\tau}$ and $\int_{\frac{1}{\Re(\sqrt{\lambda})}}^{\infty} \int_{r}^{\tau}$ and then apply (338), (339), and Lemmas A.2, A.3, which leads to

$$
\begin{aligned}
& \left|J_{20}\left[f_{n}\right](r)\right| \\
& \leq C|\lambda|^{-\frac{1}{2}} r^{|n|} \int_{r}^{\frac{1}{\Re(\sqrt{\lambda})}}|\lambda|^{\frac{\Re(\mu)+1}{2}} \tau^{\Re(\mu)+2-|n|}|\sqrt{\lambda} \tau|^{-\Re(\mu)}\left|j_{n}\right| \mathrm{d} \tau \\
& +C|\lambda|^{-\frac{1}{2}} r^{|n|} \int_{\frac{1}{\Re(\sqrt{\lambda})}}^{\infty}\left(|\lambda|^{\frac{|n|-1}{2}}+|\lambda|^{-\frac{3}{4}} \tau^{-\frac{1}{2}-|n|} e^{\tau \Re(\sqrt{\lambda})}\right)|\sqrt{\lambda} \tau|^{-\frac{1}{2}} e^{-\tau \Re(\sqrt{\lambda})}\left|j_{n}\right| \mathrm{d} \tau \\
& \leq C r^{|n|} \int_{r}^{\frac{1}{\Re(\sqrt{\lambda})}} \tau^{2-|n|}\left|j_{n}\right| \mathrm{d} \tau+C|\lambda|^{\frac{|n|}{2}-\frac{5}{4}} r^{|n|} \int_{\frac{1}{\Re(\sqrt{\lambda})}}^{\infty} \tau^{-\frac{1}{2}} e^{-\tau \Re(\sqrt{\lambda})}\left|j_{n}\right| \mathrm{d} \tau \\
& \quad+C|\lambda|^{-\frac{3}{2}} r^{|n|} \int_{\frac{1}{\Re(\sqrt{\lambda})}}^{\infty} \tau^{-1-|n|}\left|j_{n}\right| \mathrm{d} \tau
\end{aligned}
$$

Then one can derive from (144) the estimate (141) also for the case $\Re(\sqrt{\lambda}) \leq r^{-1}$ and $l=20$. 
(iv) Estimate of $J_{21}\left[f_{n}\right]$ : By the inequality

$$
\int_{\tau}^{\infty} s^{-1-|n|}\left|I_{\mu}(\sqrt{\lambda} s)\right| \mathrm{d} s \leq \tau^{-1} \int_{\tau}^{\infty} s^{-|n|}\left|I_{\mu}(\sqrt{\lambda} s)\right| \mathrm{d} s,
$$

the proof is a minor modification of the proof of the estimate for $J_{20}\left[f_{n}\right]$ above. Thus we omit the details.

(v) Estimates of $J_{22}\left[f_{n}\right]$ and $J_{23}\left[f_{n}\right]$ : We will give a proof for $J_{23}\left[f_{n}\right]$. Let $\Re(\sqrt{\lambda}) \geq r^{-1}$. Then we have from Lemma A.3,

$$
\begin{aligned}
\left|J_{23}\left[f_{n}\right](r)\right| & \leq C|\lambda|^{-\frac{1}{2}}|\sqrt{\lambda} r|^{-\frac{1}{2}} e^{r \Re(\sqrt{\lambda})} \int_{r}^{\infty}|\sqrt{\lambda} \tau|^{-\frac{1}{2}} e^{-\tau \Re(\sqrt{\lambda})}\left|f_{\theta, n}\right| \tau \mathrm{d} \tau \\
& \leq C|\lambda|^{-1} r^{-\frac{1}{2}} e^{r \Re(\sqrt{\lambda})} \int_{r}^{\infty} \tau^{\frac{1}{2}} e^{-\tau \Re(\sqrt{\lambda})}\left|f_{\theta, n}\right| \mathrm{d} \tau
\end{aligned}
$$

which yields for $\Re(\sqrt{\lambda}) \geq r^{-1}$,

$$
r^{-1}\left|J_{23}\left[f_{n}\right](r)\right| \leq \frac{C}{|\lambda|}\left\|f_{\theta, n}\right\|_{L^{\infty}(\Omega)} .
$$

If $\Re(\sqrt{\lambda}) \leq r^{-1}$ then we have from Lemma A.2,

$$
\begin{aligned}
\left|J_{23}\left[f_{n}\right](r)\right| \leq & C|\lambda|^{-\frac{1}{2}}|\sqrt{\lambda} r|^{\Re(\mu)}\left(\int_{r}^{\frac{1}{\Re(\sqrt{\lambda})}}|\sqrt{\lambda} \tau|^{-\Re(\mu)+1}\left|f_{\theta, n}\right| \tau \mathrm{d} \tau\right. \\
& \left.+\int_{\frac{1}{\Re(\sqrt{\lambda})}}^{\infty}|\sqrt{\lambda} \tau|^{-\frac{1}{2}} e^{-\tau \Re(\sqrt{\lambda})}\left|f_{\theta, n}\right| \tau \mathrm{d} \tau\right) \\
\leq & C r^{\Re(\mu)} \int_{r}^{\frac{1}{\Re(\sqrt{\lambda})}} \tau^{2-\Re(\mu)}\left|f_{\theta, n}\right| \mathrm{d} \tau \\
& +C|\lambda|^{\frac{\Re(\mu)}{2}-\frac{3}{4}} r^{\Re(\mu)} \int_{\frac{1}{\Re(\sqrt{\lambda})}}^{\infty} \tau^{\frac{1}{2}} e^{-\tau \Re(\sqrt{\lambda})}\left|f_{\theta, n}\right| \mathrm{d} \tau
\end{aligned}
$$

Hence (146) holds also in the case $\Re(\sqrt{\lambda}) \leq r^{-1}$. The estimate of $J_{22}\left[f_{n}\right]$ is obtained in the same manner, and the details are omitted here. The proof is complete.

Lemma 3.15 Let $l=24,25,26$, and let $f_{n} \in \mathcal{P}_{n} C_{0}^{\infty}(\Omega)^{2}$. Then $J_{24}\left[f_{n}\right]=0$ if $|n|=1$, and

$$
\sup _{r \geq 1} r^{-1}\left|J_{l}\left[f_{n}\right](r)\right| \leq \frac{C}{|\lambda|}\left\|f_{r, n}\right\|_{L^{\infty}(\Omega)} .
$$

Proof. (i) Estimate of $J_{24}\left[f_{n}\right]$ : It is clear that $J_{24}\left[f_{n}\right]=0$ if $|n|=1$, so we assume that $|n| \geq 2$. Firstly we observe from Lemma A.2 and Lemma A.3 that

$$
\left|L_{\mu}(\sqrt{\lambda} r)\right| \leq C|\lambda|^{-\frac{1}{2}} r^{-1}
$$

holds for all $r \geq 1$. Then we have

$$
\begin{aligned}
\left|J_{24}\left[f_{n}\right](r)\right| & \leq C|\lambda|^{-\frac{1}{2}} r^{|n|} \int_{r}^{\infty} \tau^{1-|n|}|\lambda|^{-\frac{1}{2}} \tau^{-1}\left|f_{r, n}\right| \mathrm{d} \tau \\
& \leq C|\lambda|^{-1} r\left\|f_{r, n}\right\|_{L^{\infty}(\Omega)}
\end{aligned}
$$


where $|n| \geq 2$ is used.

(ii) Estimate of $J_{25}\left[f_{n}\right]$ : From (149) we have

$$
\left|J_{25}\left[f_{n}\right](r)\right| \leq C|\lambda|^{-1} r\left|f_{r, n}(r)\right| .
$$

Hence the results are straightforward. In particular, we have $J_{25}\left[f_{n}\right](1)=0$ if $f_{n} \in$ $C_{0}^{\infty}(\Omega)^{2}$.

(iii) Estimate of $J_{26}\left[f_{n}\right]$ : Firstly we observe from (327) that

$$
\begin{aligned}
L_{\mu}(z) & =\left(-\frac{\mu}{z} K_{\mu}(z)-\frac{\mathrm{d} K_{\mu}}{\mathrm{d} z}(z)\right) I_{\mu}(z)+K_{\mu}(z)\left(-\frac{\mu}{z} I_{\mu}(z)+\frac{\mathrm{d} I_{\mu}}{\mathrm{d} z}(z)\right) \\
& =-\frac{2 \mu}{z} K_{\mu}(z) I_{\mu}(z)+K_{\mu}(z) \frac{\mathrm{d} I_{\mu}}{\mathrm{d} z}(z)-\frac{\mathrm{d} K_{\mu}}{\mathrm{d} z}(z) I_{\mu}(z) \\
& =-\frac{2 \mu}{z} K_{\mu}(z) I_{\mu}(z)+\frac{1}{z} .
\end{aligned}
$$

Therefore, we see

$$
\begin{aligned}
& \frac{\mathrm{d}}{\mathrm{d} z}\left(z^{2-|n|} L_{\mu}(z)\right)=\frac{\mathrm{d}}{\mathrm{d} z}\left(z^{1-|n|}\left(-2 \mu K_{\mu}(z) I_{\mu}(z)+1\right)\right) \\
& =(1-|n|) z^{-|n|}\left(-2 \mu K_{\mu}(z) I_{\mu}(z)+1\right)-2 \mu|z|^{1-|n|} \frac{\mathrm{d}}{\mathrm{d} z}\left(K_{\mu}(z) I_{\mu}(z)\right),
\end{aligned}
$$

while

$$
\begin{aligned}
\frac{\mathrm{d}}{\mathrm{d} z}\left(K_{\mu}(z) I_{\mu}(z)\right) & =\left(-\frac{\mu}{z} K_{\mu}(z)-K_{\mu-1}(z)\right) I_{\mu}(z)+K_{\mu}(z)\left(\frac{\mu}{z} I_{\mu}(z)+I_{\mu+1}(z)\right) \\
& =-K_{\mu-1}(z) I_{\mu}(z)+K_{\mu}(z) I_{\mu+1}(z) .
\end{aligned}
$$

Then Lemma A.2 implies

$$
\left|\frac{\mathrm{d}}{\mathrm{d} z}\left(K_{\mu}(z) I_{\mu}(z)\right)\right| \leq C|z|
$$

for any $|z| \leq M$ with $C$ depending only on $M$ and $\mu$, while for $z \mid \gg 1$ we have from (154) and the expansion (330),

$$
\left|\frac{\mathrm{d}}{\mathrm{d} z}\left(K_{\mu}(z) I_{\mu}(z)\right)\right| \leq C|z|^{-2} .
$$

Thus, the identity (153) together with the estimates (155) and (156) yields

$$
\begin{array}{ll}
\left|\frac{\mathrm{d}}{\mathrm{d} z}\left(z^{2-|n|} L_{\mu}(z)\right)\right| \leq C\left((1-|n|)|z|^{-|n|}+|z|^{2-|n|}\right), & |z| \leq 1, \\
\left|\frac{\mathrm{d}}{\mathrm{d} z}\left(z^{2-|n|} L_{\mu}(z)\right)\right| \leq C\left((1-|n|)|z|^{-|n|}+|z|^{-1-|n|}\right), & |z|>1 .
\end{array}
$$

Therefore, if $\Re(\sqrt{\lambda}) \geq r^{-1}$ then (157) yields

$$
\begin{aligned}
& \left|J_{26}\left[f_{n}\right](r)\right| \\
& \leq C|\lambda|^{-1+\frac{|n|}{2}} r^{|n|} \int_{r}^{\infty}\left((1-|n|)|\sqrt{\lambda} \tau|^{-|n|}+|\sqrt{\lambda} \tau|^{-1-|n|}\right)\left|f_{r, n}\right| \mathrm{d} \tau \\
& \leq C|\lambda|^{-1} r\left\|f_{r, n}\right\|_{L^{\infty}(\Omega)} .
\end{aligned}
$$


Here we have used the fact that the coefficient $1-|n|$ is zero when $|n|=1$. In the case $\Re(\sqrt{\lambda}) \leq r^{-1}$ we decompose the integral into $\int_{r}^{\frac{1}{\Re(\sqrt{\lambda})}}$ and $\int_{\frac{1}{\Re(\sqrt{\lambda})}}^{\infty}$, and then by applying (157),

$$
\begin{aligned}
& \left|J_{26}\left[f_{n}\right](r)\right| \\
& \leq C|\lambda|^{-1+\frac{|n|}{2}} r^{|n|}\left(\int_{r}^{\frac{1}{\Re(\sqrt{\lambda})}}\left((1-|n|)|\sqrt{\lambda} \tau|^{-|n|}+|\sqrt{\lambda} \tau|^{2-|n|}\right)\left|f_{r, n}\right| \mathrm{d} \tau\right. \\
& \left.\quad+\int_{\frac{1}{\Re(\sqrt{\lambda})}}^{\infty}\left((1-|n|)|\sqrt{\lambda} \tau|^{-|n|}+|\sqrt{\lambda} \tau|^{-1-|n|}\right)\left|f_{r, n}\right| \mathrm{d} \tau\right) \\
& \leq C|\lambda|^{-1} r^{|n|}\left((1-|n|) r^{1-|n|}+|\lambda| \int_{r}^{\frac{1}{\Re(\sqrt{\lambda})}} \tau^{2-|n|} \mathrm{d} \tau+|\lambda|^{\frac{|n|-1}{2}}\right)\left\|f_{r, n}\right\|_{L^{\infty}(\Omega)},
\end{aligned}
$$

which implies $r^{-1}\left|J_{26}\left[f_{n}\right](r)\right| \leq C|\lambda|^{-1}\left\|f_{r, n}\right\|_{L^{\infty}(\Omega)}$ also for $\Re(\sqrt{\lambda}) \leq r^{-1}$. The proof is complete.

We are now in position to establish the estimates for $V_{n}\left[\Phi_{n, \lambda}\left[f_{n}\right]\right]$ and $c_{n, \lambda}\left[f_{n}\right]$. Note that we always assume $\alpha \neq 0$, thus, $\Re\left(\mu_{n}(\alpha)\right)>|n|$ holds and the estimates we have established so far can be applied.

Proposition 3.16 Let $1 \leq q<p \leq \infty$ or $1<q \leq p \leq \infty$, and let $\lambda \in \Sigma_{\pi-\epsilon}$ for some $\epsilon \in\left(0, \frac{\pi}{2}\right)$. Then there is a positive constant $C=C(\alpha, n, \epsilon, p, q)$ such that, for any $f_{n} \in \mathcal{P}_{n} C_{0, \sigma}^{\infty}(\Omega)$,

$$
\begin{aligned}
\left\|V_{n}\left[\Phi_{n, \lambda}\left[f_{n}\right]\right]\right\|_{L^{p}(\Omega)} & \leq \frac{C}{|\lambda|^{1+\frac{1}{p}-\frac{1}{q}}}\left\|f_{n}\right\|_{L^{q}(\Omega)}, \\
\left|c_{n, \lambda}\left[f_{n}\right]\right| & \leq \frac{C}{|\lambda|^{1-\frac{1}{q}}\left(1+|\lambda|^{\frac{1}{2 q}}\right)}\left\|f_{n}\right\|_{L^{q}(\Omega)} .
\end{aligned}
$$

Proof. Set $w_{n}=\Phi_{n, \lambda}\left[f_{n}\right]$. Firstly we recall that $V_{n}\left[w_{n}\right]=V_{r, n}\left[w_{n}\right] e^{i n \theta} \mathbf{e}_{r}+V_{\theta, n}\left[w_{n}\right] e^{i n \theta} \mathbf{e}_{\theta}$, where $V_{r, n}\left[w_{n}\right]=\frac{i n}{r} \psi_{n}\left[w_{n}\right]$ and $V_{\theta, n}\left[w_{n}\right]=-\frac{\mathrm{d}}{\mathrm{d} r} \psi_{n}\left[w_{n}\right]$. Thus, by the definition of $\psi_{n}\left[w_{n}\right]$, we see

$$
\begin{aligned}
V_{r, n}\left[w_{n}\right](r)= & \frac{i n}{2|n| r}\left(\frac{1}{r^{|n|}}\left(-c_{n, \lambda}\left[f_{n}\right]+\int_{1}^{r} s^{1+|n|} \Phi_{n, \lambda}\left[f_{n}\right](s) \mathrm{d} s\right)\right. \\
& \left.+r^{|n|} \int_{r}^{\infty} s^{1-|n|} \Phi_{n, \lambda}\left[f_{n}\right](s) \mathrm{d} s\right)
\end{aligned}
$$

and

$$
\begin{aligned}
V_{\theta, n}\left[w_{n}\right](r)= & -\frac{1}{2 r}\left(\frac{1}{r^{|n|}}\left(-c_{n, \lambda}\left[f_{n}\right]+\int_{1}^{r} s^{1+|n|} \Phi_{n, \lambda}\left[f_{n}\right](s) \mathrm{d} s\right)\right. \\
& \left.-r^{|n|} \int_{r}^{\infty} s^{1-|n|} \Phi_{n, \lambda}\left[f_{n}\right](s) \mathrm{d} s\right) .
\end{aligned}
$$

Here we recall that

$$
c_{n, \lambda}\left[f_{n}\right]=\int_{1}^{\infty} s^{1-|n|} \Phi_{n, \lambda}\left[f_{n}\right](s) \mathrm{d} s .
$$


Then Lemma 3.6 and Lemma 3.9 show that

$$
\begin{aligned}
I\left[f_{n}\right](r) & :=\frac{1}{r^{|n|+1}}\left(-c_{n, \lambda}\left[f_{n}\right]+\int_{1}^{r} s^{1+|n|} \Phi_{n, \lambda}\left[f_{n}\right](s) \mathrm{d} s\right) \\
& =-\frac{1}{r^{|n|+1}} \sum_{l=10}^{17} J_{l}\left[f_{n}\right](1)+\frac{1}{r} \sum_{l=1}^{9} J_{l}\left[f_{n}\right](r) \\
& =-\frac{1}{r^{|n|+1}} \sum_{l=11,13,14,15} J_{l}\left[f_{n}\right](1)+\frac{1}{r} \sum_{l=1}^{8} J_{l}\left[f_{n}\right](r),
\end{aligned}
$$

by the cancellation $-r^{-|n|} J_{17}\left[f_{n}\right](1)+J_{9}\left[f_{n}\right](r)=0$ and $J_{10}\left[f_{n}\right](1)=J_{12}\left[f_{n}\right](1)=$ $J_{16}\left[f_{n}\right](1)=0$ by their definitions. Then we have from Corollary 3.12 (i),

$$
\left\|r^{-1} \sum_{l=1}^{8} J_{l}\left[f_{n}\right]\right\|_{L^{p}(\Omega)} \leq \frac{C}{|\lambda|^{1+\frac{1}{p}-\frac{1}{q}}}\left\|f_{n}\right\|_{L^{q}(\Omega)}, \quad 1 \leq q \leq p \leq \infty,
$$

for any $\lambda \in \Sigma_{\pi-\epsilon}$ with $\epsilon \in\left(0, \frac{\pi}{2}\right)$. As for the other terms in (165), we have from Corollary 3.12 (iii),

$$
\left\|\frac{1}{r^{|n|+1}} \sum_{l=11,13,14,15} J_{l}\left[f_{n}\right](1)\right\|_{L^{p}(\Omega)} \leq \frac{C}{|\lambda|^{1-\frac{1}{q}}\left(1+|\lambda|^{\frac{1}{q}}\right)}\left\|f_{n}\right\|_{L^{q}(\Omega)},
$$

for $1 \leq q<p \leq \infty$ or $1<q \leq p<\infty$. Hence, (166) and (167) gives

$$
\left\|I\left[f_{n}\right]\right\|_{L^{p}(\Omega)} \leq \frac{C}{|\lambda|^{1+\frac{1}{p}-\frac{1}{q}}}\left\|f_{n}\right\|_{L^{q}(\Omega)}
$$

for $1 \leq q<p \leq \infty$ or $1<q \leq p<\infty$. Next we have from Lemma 3.9,

$$
I I\left[f_{n}\right](r):=r^{|n|-1} \int_{r}^{\infty} s^{1-|n|} \Phi_{n, \lambda}\left[f_{n}\right](s) \mathrm{d} s=r^{-1} \sum_{l=10}^{17} J_{l}\left[f_{n}\right](r) .
$$

Hence, Corollary 3.12 (i) and (ii) imply that

$$
\left\|I I\left[f_{n}\right]\right\|_{L^{p}(\Omega)} \leq \frac{C}{|\lambda|^{1+\frac{1}{p}-\frac{1}{q}}}\left\|f_{n}\right\|_{L^{q}(\Omega)}
$$

for $1 \leq q<p \leq \infty$ or $1<q \leq p<\infty$. Combining (168) and (170) with the expressions (163) and (164), we have proved (161) expect for the case $p=q=\infty$. Since $c_{n, \lambda}\left[f_{n}\right]=$ $I I\left[f_{n}\right](1)$, the estimate (162) with $1 \leq q<\infty$ and $\Re(\sqrt{\lambda}) \leq 1$ follows from (170) with $p=\infty$. On the other hand, if $\Re(\sqrt{\lambda}) \geq 1$ and $1 \leq q<\infty$ then from $I I\left[f_{n}\right](1)=$ $\sum_{l=10}^{17} J_{l}\left[f_{n}\right](1)=\sum_{l=11,13,14,15} J_{l}\left[f_{n}\right](1)+J_{17}\left[f_{n}\right](1)$ and (167) we have

$$
\left|c_{n, \lambda}\left[f_{n}\right]\right| \leq \frac{C}{|\lambda|}\left\|f_{n}\right\|_{L^{q}(\Omega)}+\left|J_{17}\left[f_{n}\right](1)\right| .
$$

Noting $\left|J_{17}\left[f_{n}\right](1)\right|=\left|J_{9}\left[f_{n}\right](1)\right|$ by their definitions, we use Lemma 3.7 (3) and the interpolation theorem, which leads to

$$
\left|J_{17}\left[f_{n}\right](1)\right| \leq \frac{C}{|\lambda|^{1-\frac{1}{2 q}}}\left\|f_{n}\right\|_{L^{q}(\Omega)} .
$$


Hence (162) has been proved for $1 \leq q<\infty$.

It remains to show (161) for $p=q=\infty$ and (162) for $q=\infty$. Firstly let us go back to (165). Then we apply Lemma 3.13 to rewrite

$$
\sum_{l=11,13,14,15} J_{l}\left[f_{n}\right](1)=\sum_{j=18}^{26} J_{l}\left[f_{n}\right](1)=\sum_{j=18}^{24} J_{l}\left[f_{n}\right](1)+J_{26}\left[f_{n}\right](1),
$$

where we have used $J_{25}\left[f_{n}\right](1)=0$ due to the condition $f_{n} \in C_{0}^{\infty}(\Omega)^{2}$. Then Lemma 3.14 and Lemma 3.15 show

$$
\left|J_{l}\left[f_{n}\right](1)\right| \leq \frac{C}{|\lambda|}\left\|f_{n}\right\|_{L^{\infty}(\Omega)}, \quad l=18, \cdots, 26 .
$$

Collecting (165), (166), and (174), we obtain

$$
\left\|I\left[f_{n}\right]\right\|_{L^{\infty}(\Omega)} \leq \frac{C}{|\lambda|}\left\|f_{n}\right\|_{L^{\infty}(\Omega)} .
$$

Next we have from Lemma 3.9 and Lemma 3.13,

$$
\begin{aligned}
I I\left[f_{n}\right](r) & :=r^{|n|-1} \int_{r}^{\infty} s^{1-|n|} \Phi_{n, \lambda}\left[f_{n}\right](s) \mathrm{d} s \\
& =r^{-1}\left(J_{10}\left[f_{n}\right](r)+J_{12}\left[f_{n}\right](r)+J_{16}\left[f_{n}\right](r)+J_{17}\left[f_{n}\right](r)+\sum_{l=18}^{26} J_{l}\left[f_{n}\right](r)\right) .
\end{aligned}
$$

Then Lemmas 3.10, 3.14, and 3.15 show that each $r^{-1} J_{l}[\cdot]$ in (170) is bounded from $L^{\infty}(\Omega)^{2}$ to $L^{\infty}(\Omega)$ with the estimate $C|\lambda|^{-1}$ of its operator norm. Thus we have

$$
\left\|I I\left[f_{n}\right]\right\|_{L^{\infty}(\Omega)} \leq \frac{C}{|\lambda|}\left\|f_{n}\right\|_{L^{\infty}(\Omega)} .
$$

Thus, (175) and (177) together with the expressions (163) and (164) lead to

$$
\left\|V_{n}\left[\Phi_{n, \lambda}\left[f_{n}\right]\right]\right\|_{L^{\infty}(\Omega)} \leq \frac{C}{|\lambda|}\left\|f_{n}\right\|_{L^{\infty}(\Omega)},
$$

as desired. Finally, the estimate (162) for the case $q=\infty$ follows from (177). The proof is complete.

Recalling the structure of the resolvent in (79), we also need to estimate $V_{n}\left[K_{\mu_{n}}(\sqrt{\lambda} \cdot)\right]$ and $F_{n}(\sqrt{\lambda} ; \alpha)$. We start from the estimate of $V_{n}\left[K_{\mu_{n}}(\sqrt{\lambda} \cdot)\right]$.

Proposition 3.17 Let $1<p \leq \infty$ and let $\lambda \in \Sigma_{\pi-\epsilon}$ for some $\epsilon \in\left(0, \frac{\pi}{2}\right)$. Then there is a positive constant $C=C(\alpha, n, \epsilon, p)$ such that the following statements hold. If $\Re(\sqrt{\lambda}) \leq 1$ then

$$
\left\|V_{n}\left[K_{\mu_{n}}(\sqrt{\lambda} \cdot)\right]\right\|_{L^{p}(\Omega)} \leq \frac{C}{|\lambda|^{\frac{\Re\left(\mu_{n}\right)}{2}+\frac{1}{p}}},
$$

while if $\Re(\sqrt{\lambda}) \geq 1$ then

$$
\left\|V_{n}\left[K_{\mu_{n}}(\sqrt{\lambda} \cdot)\right]\right\|_{L^{p}(\Omega)} \leq \frac{C e^{-\Re(\sqrt{\lambda})}}{|\lambda|^{\frac{3}{4}+\frac{1}{2 p}}} .
$$


Proof. (i) Let $\Re(\sqrt{\lambda}) \leq 1$. If $\Re(\sqrt{\lambda}) \leq r^{-1}$ then we have from (333) and (335) together with $\Re(\mu)>|n|$,

$$
r^{-|n|-1} \int_{1}^{r} s^{1+|n|}\left|K_{\mu_{n}}(\sqrt{\lambda} s)\right| \mathrm{d} s \leq C|\lambda|^{-\frac{\Re\left(\mu_{n}\right)}{2}} r^{1-|n|},
$$

and

$$
r^{|n|-1} \int_{r}^{\infty} s^{1-|n|}\left|K_{\mu_{n}}(\sqrt{\lambda} s)\right| \mathrm{d} s \leq C|\lambda|^{-\frac{\Re\left(\mu_{n}\right)}{2}} r^{1-\Re\left(\mu_{n}\right)} \leq C|\lambda|^{-\frac{\Re\left(\mu_{n}\right)}{2}} r^{1-|n|} .
$$

In particular, we have

$$
\left|d_{n}\left[K_{\mu_{n}}(\sqrt{\lambda} \cdot)\right]\right| \leq \int_{1}^{\infty} s^{1-|n|}\left|K_{\mu_{n}}(\sqrt{\lambda} s)\right| \mathrm{d} s \leq C|\lambda|^{-\frac{\Re\left(\mu_{n}\right)}{2}},
$$

and the definition of $V_{n}\left[K_{\mu_{n}}(\sqrt{\lambda} \cdot)\right]$ implies that for $1 \leq p \leq \infty$,

$$
\begin{aligned}
& \sup _{1 \leq|x| \leq \frac{1}{\Re(\sqrt{\lambda})}}|x|^{\frac{2}{p}}\left|V_{n}\left[K_{\mu_{n}}(\sqrt{\lambda} \cdot)\right](x)\right| \\
& \leq C \sup _{1 \leq r \leq \frac{1}{\Re(\sqrt{\lambda})}} r^{\frac{2}{p}}\left(r^{-|n|-1}\left|d_{n}\left[K_{\mu_{n}}(\sqrt{\lambda} \cdot)\right]\right|+r^{-|n|-1} \int_{1}^{r} s^{1+|n|}\left|K_{\mu_{n}}(\sqrt{\lambda} s)\right| \mathrm{d} s\right. \\
& \left.+r^{|n|-1} \int_{r}^{\infty} s^{1-|n|}\left|K_{\mu_{n}}(\sqrt{\lambda} s)\right| \mathrm{d} s\right) \\
& \leq C|\lambda|^{-\frac{\Re\left(\mu_{n}\right)}{2}-\frac{1}{p}} \text {. }
\end{aligned}
$$

On the other hand, if $1 \geq \Re(\sqrt{\lambda})>r^{-1}$ then we have from (332) and (334),

$$
\begin{aligned}
r^{-|n|-1} \int_{1}^{r} s^{1+|n|}\left|K_{\mu_{n}}(\sqrt{\lambda} s)\right| \mathrm{d} s & \leq C r^{-|n|-1}\left(|\lambda|^{-1-\frac{|n|}{2}}+|\lambda|^{-\frac{\Re\left(\mu_{n}\right)}{2}-1}\right) \\
& \leq C|\lambda|^{-1-\frac{\Re\left(\mu_{n}\right)}{2}} r^{-|n|-1},
\end{aligned}
$$

and

$$
r^{|n|-1} \int_{r}^{\infty} s^{1-|n|}\left|K_{\mu_{n}}(\sqrt{\lambda} s)\right| \mathrm{d} s \leq C|\lambda|^{-\frac{3}{4}} r^{-\frac{1}{2}} e^{-r \Re(\sqrt{\lambda})} \leq C|\lambda|^{-\frac{1}{2}} e^{-r \Re(\sqrt{\lambda})} .
$$

Hence, as in the calculation in (184), the estimates (183), (185), and (186) yield for $1 \leq$ $p \leq \infty$,

$$
\begin{aligned}
& \sup _{\frac{1}{\Re(\sqrt{\lambda})} \leq|x|}|x|^{\frac{2}{p}}\left|V_{n}\left[K_{\mu_{n}}(\sqrt{\lambda} \cdot)\right](x)\right| \\
& \leq C \sup _{\frac{1}{\Re(\sqrt{\lambda})} \leq r}\left(\left|d_{n}\left[K_{\mu_{n}}(\sqrt{\lambda} \cdot)\right]\right|+|\lambda|^{-1-\frac{\Re\left(\mu_{n}\right)}{2}} r^{\frac{2}{p}-|n|-1}+|\lambda|^{-\frac{1}{2}} r^{\frac{2}{p}} e^{-r \Re(\sqrt{\lambda})}\right) \\
& \leq C|\lambda|^{-\frac{\Re\left(\mu_{n}\right)}{2}-\frac{1}{p}}
\end{aligned}
$$

where $\Re\left(\mu_{n}\right)>|n| \geq 1$ and $\frac{2}{p}-|n|-1 \leq 0$ are used. By the interpolation theorem the estimate (179) follows from (184) and (187). The proof is complete for the case $\Re(\sqrt{\lambda}) \leq 1$. 
(ii) Let $\Re(\sqrt{\lambda}) \geq 1$. In order to obtain the factor $|\lambda|^{-\frac{1}{2 p}}$ in (180) we need to rewrite the terms of $V_{n}\left[K_{\mu_{n}}(\sqrt{\lambda} \cdot)\right]$ suitably by using Lemma A.1. In view of the definition of $V_{n}\left[K_{\mu_{n}}(\sqrt{\lambda} \cdot)\right]$ it suffices to the estimates the terms

$$
\begin{aligned}
I(r) & =\frac{1}{r^{|n|+1}}\left(-\int_{1}^{\infty} s^{1-|n|} K_{\mu_{n}}(\sqrt{\lambda} s) \mathrm{d} s+\int_{1}^{r} s^{1+|n|} K_{\mu_{n}}(\sqrt{\lambda} s) \mathrm{d} s\right), \\
I I(r) & =r^{|n|-1} \int_{r}^{\infty} s^{1-|n|} K_{\mu_{n}}(\sqrt{\lambda} s) \mathrm{d} s .
\end{aligned}
$$

We apply the identity $K_{\mu_{n}}(z)=-\frac{\mu_{n}+1}{z} K_{\mu_{n}+1}(z)-\frac{\mathrm{d} K_{\mu_{n}+1}}{\mathrm{~d} z}(z)$, see (327), to $I$, which leads to

$$
\begin{aligned}
I(r)= & -\frac{\mu_{n}+1}{\sqrt{\lambda} r|n|+1}\left(-\int_{1}^{\infty} s^{-|n|} K_{\mu_{n}+1}(\sqrt{\lambda} s) \mathrm{d} s+\int_{1}^{r} s^{|n|} K_{\mu_{n}+1}(\sqrt{\lambda} s) \mathrm{d} s\right) \\
& -\frac{1}{\sqrt{\lambda} r|n|+1}\left(-\int_{1}^{\infty} s^{1-|n|} \frac{\mathrm{d}}{\mathrm{d} s} K_{\mu_{n}+1}(\sqrt{\lambda} s) \mathrm{d} s+\int_{1}^{r} s^{1+|n|} \frac{\mathrm{d}}{\mathrm{d} s} K_{\mu_{n}+1}(\sqrt{\lambda} s) \mathrm{d} s\right) \\
= & -\frac{1}{\sqrt{\lambda} r|n|+1}\left(-\left(\mu_{n}+|n|\right) \int_{1}^{\infty} s^{-|n|} K_{\mu_{n}+1}(\sqrt{\lambda} s) \mathrm{d} s\right. \\
& \left.+\left(\mu_{n}-|n|\right) \int_{1}^{r} s^{|n|} K_{\mu_{n}+1}(\sqrt{\lambda} s) \mathrm{d} s\right)-\frac{1}{\sqrt{\lambda}} K_{\mu_{n}+1}(\sqrt{\lambda} r)
\end{aligned}
$$

Similarly, the term $I I$ is rewritten as

$$
I I(r)=-\frac{\mu_{n}+|n|}{\sqrt{\lambda}} r^{|n|-1} \int_{r}^{\infty} s^{-|n|} K_{\mu_{n}+1}(\sqrt{\lambda} s) \mathrm{d} s+\frac{1}{\sqrt{\lambda}} K_{\mu_{n}+1}(\sqrt{\lambda} r) .
$$

Therefore, we have from Lemma A.3,

$$
\begin{aligned}
|I(r)| & \leq \frac{C}{|\lambda|^{\frac{1}{2}} r^{|n|+1}} \int_{1}^{\infty} s^{|n|}|\sqrt{\lambda} s|^{-\frac{1}{2}} e^{-s \Re(\sqrt{\lambda})} \mathrm{d} s+\frac{C}{|\lambda|^{\frac{1}{2}}}|\sqrt{\lambda} r|^{-\frac{1}{2}} e^{-r \Re(\sqrt{\lambda})} \\
& \leq \frac{C}{|\lambda|^{\frac{5}{4}} r^{|n|+1}} e^{-\Re(\sqrt{\lambda})}+\frac{C}{|\lambda|^{\frac{3}{4}} r^{\frac{1}{2}}} e^{-r \Re(\sqrt{\lambda})}
\end{aligned}
$$

and similarly,

$$
|I I(r)| \leq \frac{C}{|\lambda|^{\frac{5}{4}} r^{\frac{3}{2}}} e^{-r \Re(\sqrt{\lambda})}+\frac{C}{|\lambda|^{\frac{3}{4}} r^{\frac{1}{2}}} e^{-r \Re(\sqrt{\lambda})} \leq \frac{C}{|\lambda|^{\frac{3}{4}} r^{\frac{1}{2}}} e^{-r \Re(\sqrt{\lambda})} .
$$

Since the $L^{p}(\Omega)$ norm of the function $r^{-\frac{1}{2}} e^{-r \Re(\sqrt{\lambda})}$ (note that we regard it as a radially symmetric function in $\Omega$, as before) is bounded from above by $C|\lambda|^{-\frac{1}{2 p}} e^{-\Re(\sqrt{\lambda})}$ when $\Re(\sqrt{\lambda}) \geq 1$, we have for $1<p \leq \infty$,

$$
\left\|V_{n}\left[K_{\mu_{n}}(\sqrt{\lambda} \cdot)\right]\right\|_{L^{p}(\Omega)} \leq C\|I\|_{L^{p}(\Omega)}+C\|I I\|_{L^{p}(\Omega)} \leq \frac{C}{|\lambda|^{\frac{3}{4}+\frac{1}{2 p}}} e^{-\Re(\sqrt{\lambda})} .
$$

Here we have used $|\lambda|^{-\frac{5}{4}} \leq|\lambda|^{-\frac{3}{4}-\frac{1}{2 p}}$ for $\Re(\sqrt{\lambda}) \geq 1$ and $1 \leq p \leq \infty$. The proof is complete.

We have now proved the boundedness of $T_{n, \alpha}$ and $R_{n, \alpha}$ as follows, which completes the proof of Proposition 3.4. 
Corollary 3.18 The maps $T_{n, \alpha}, R_{n, \alpha}: \mathbb{C} \backslash \overline{\mathbb{R}_{-}} \rightarrow \mathcal{L}\left(\mathcal{P}_{n} L_{\sigma}^{2}(\Omega)\right)$ defined as (75) and (76) are locally bounded.

Proof. The assertion is a direct consequence of Propositions 3.16 and 3.17 by the density argument, for $T_{n, \alpha}(\lambda) f_{n}=-c_{n, \lambda}\left[f_{n}\right] V_{n}\left[K_{\mu_{n}}(\sqrt{\lambda} \cdot)\right]$ and $R_{n, \alpha}(\lambda)=V_{n}\left[\Phi_{n, \lambda}\left[f_{n}\right]\right]$. The proof is complete.

Collecting Propositions 3.16, 3.17, and the formula (79), we obtain the $L^{p}-L^{q}$ estimates for the resolvent as follows.

Theorem 3.19 Let $1 \leq q<p \leq \infty$ or $1<q \leq p \leq \infty$. Let $\lambda \in \rho\left(-\left.A_{\alpha}\right|_{\mathcal{P}_{n} L_{\sigma}^{2}}\right) \cap \Sigma_{\pi-\epsilon}$ for some $\epsilon \in\left(0, \frac{\pi}{2}\right)$. Then there is a positive constant $C=C(\alpha, n, \epsilon, p, q)$ such that the following statements hold. Let $f_{n} \in \mathcal{P}_{n} C_{0, \sigma}^{\infty}(\Omega)$. If $\Re(\sqrt{\lambda}) \leq 1$ then

$$
\left\|\left(\lambda+\left.A_{\alpha}\right|_{\mathcal{P}_{n} L_{\sigma}^{2}}\right)^{-1} f_{n}\right\|_{L^{p}(\Omega)} \leq \frac{C}{|\lambda|^{1+\frac{1}{p}-\frac{1}{q}}}\left(\frac{|\lambda|^{-\frac{\Re(\mu)}{2}}}{\left|F_{n}(\sqrt{\lambda} ; \alpha)\right|}+1\right)\left\|f_{n}\right\|_{L^{q}(\Omega)},
$$

while if $\Re(\sqrt{\lambda}) \geq 1$ then

$$
\left\|\left(\lambda+\left.A_{\alpha}\right|_{\mathcal{P}_{n} L_{\sigma}^{2}}\right)^{-1} f_{n}\right\|_{L^{p}(\Omega)} \leq \frac{C^{\prime}}{|\lambda|^{1+\frac{1}{p}-\frac{1}{q}}}\left(\frac{|\lambda|^{-\frac{3}{4}+\frac{1}{2 p}-\frac{1}{2 q}} e^{-\Re(\sqrt{\lambda})}}{\left|F_{n}(\sqrt{\lambda} ; \alpha)\right|}+1\right)\left\|f_{n}\right\|_{L^{q}(\Omega)} .
$$

In particular, (194) and (195) hold for all $f_{n} \in{\overline{\mathcal{P}_{n} C_{0, \sigma}^{\infty}(\Omega)}}^{\|\cdot\|_{L^{q}(\Omega)}}$.

Remark 3.20 For $1 \leq q<\infty$ we have

$$
\overline{\mathcal{P}_{n} C_{0, \sigma}^{\infty}(\Omega)} \|^{\|\cdot\|_{L} q(\Omega)}=\mathcal{P}_{n} L_{\sigma}^{q}(\Omega)=\left\{f \in L_{\sigma}^{q}(\Omega) \mid f=\mathcal{P}_{n} f\right\} .
$$

Proof of Theorem 3.19. As is stated in the proof of Proposition 3.4, we have for any $f_{n} \in$ $\mathcal{P}_{n} C_{0, \sigma}^{\infty}(\Omega)$,

$$
\left(\lambda+\left.A_{\alpha}\right|_{\mathcal{P}_{n} L_{\sigma}^{2}}\right)^{-1} f_{n}=-\frac{c_{n, \lambda}\left[f_{n}\right]}{F_{n}(\sqrt{\lambda} ; \alpha)} V_{n}\left[K_{\mu_{n}}(\sqrt{\lambda} \cdot)\right]+V_{n}\left[\Phi_{n, \lambda}\left[f_{n}\right]\right] .
$$

Let $\Re(\sqrt{\lambda}) \leq 1$. Then Propositions 3.16 and 3.17 yield

$$
\begin{aligned}
\left\|\frac{c_{n, \lambda}\left[f_{n}\right]}{F_{n}(\sqrt{\lambda} ; \alpha)} V_{n}\left[K_{\mu_{n}}(\sqrt{\lambda} \cdot)\right]\right\|_{L^{p}(\Omega)} & \leq \frac{\left|c_{n, \lambda}\left[f_{n}\right]\right|}{\left|F_{n}(\sqrt{\lambda} ; \alpha)\right|}\left\|V_{n}\left[K_{\mu_{n}}(\sqrt{\lambda} \cdot)\right]\right\|_{L^{p}(\Omega)} \\
& \leq \frac{C}{\left|F_{n}(\sqrt{\lambda} ; \alpha)\right|}|\lambda|^{-1+\frac{1}{q}-\frac{\Re\left(\mu_{n}\right)}{2}-\frac{1}{p}}\left\|f_{n}\right\|_{L^{q}(\Omega)},
\end{aligned}
$$

and

$$
\left\|V_{n}\left[\Phi_{n, \lambda}\left[f_{n}\right]\right]\right\|_{L^{p}(\Omega)} \leq \frac{C}{|\lambda|^{1+\frac{1}{p}-\frac{1}{q}}}\left\|f_{n}\right\|_{L^{q}(\Omega)} .
$$

Thus the estimate (194) holds. The estimate (195) also follows from Propositions 3.16 and 3.17 in the similar manner. The proof is complete. 


\subsubsection{Estimates for vorticity}

In order to solve the nonlinear problem $\left(\mathrm{INS}_{\alpha}\right)$ we need the derivative estimate for the resolvent $\left(\lambda+\left.A_{\alpha}\right|_{\mathcal{P}_{n} L_{\sigma}^{2}}\right)^{-1}$. By the divergence free condition and the no-slip boundary condition it suffices to study the estimates for the vorticity. To this end we first estimate $\Phi_{n, \lambda}\left[f_{n}\right]$.

Lemma 3.21 Let $\lambda \in \Sigma_{\pi-\epsilon}$ for some $\epsilon \in\left(0, \frac{\pi}{2}\right)$ and let $f_{n} \in \mathcal{P}_{n} C_{0, \sigma}^{\infty}(\Omega)$. Then it follows that

$$
\left\|\Phi_{n, \lambda}\left[f_{n}\right]\right\|_{L^{p}(\Omega)} \leq \frac{C}{|\lambda|^{\frac{1}{2}}}\left\|f_{n}\right\|_{L^{p}(\Omega)}, \quad 1 \leq p \leq \infty .
$$

Here the constant $C$ depends only on $\alpha, n, \epsilon$, and $p$.

Proof. The definition (58) for $\Phi_{n, \lambda}\left[f_{n}\right]$ leads to a decomposition $\Phi_{n, \lambda}\left[f_{n}\right]=\sum_{l=1}^{4} \Phi_{n, \lambda}^{(l)}\left[f_{n}\right]$, where

$$
\begin{aligned}
& \Phi_{n, \lambda}^{(1)}\left[f_{n}\right](r)=-K_{\mu_{n}}(\sqrt{\lambda} r) \int_{1}^{r} I_{\mu_{n}}(\sqrt{\lambda} s) g_{n} \mathrm{~d} s, \\
& \Phi_{n, \lambda}^{(2)}\left[f_{n}\right](r)=-\sqrt{\lambda} K_{\mu_{n}}(\sqrt{\lambda} r) \int_{1}^{r} I_{\mu_{n}+1}(\sqrt{\lambda} s) f_{\theta, n} s \mathrm{~d} s, \\
& \Phi_{n, \lambda}^{(3)}\left[f_{n}\right](r)=I_{\mu_{n}}(\sqrt{\lambda} r) \int_{r}^{\infty} K_{\mu_{n}}(\sqrt{\lambda} s) j_{n} \mathrm{~d} s, \\
& \Phi_{n, \lambda}^{(4)}\left[f_{n}\right](r)=\sqrt{\lambda} I_{\mu_{n}}(\sqrt{\lambda} r) \int_{r}^{\infty} K_{\mu_{n}-1}(\sqrt{\lambda} s) f_{\theta, n} s \mathrm{~d} s .
\end{aligned}
$$

(i) Estimate of $\Phi_{n, \lambda}^{(1)}\left[f_{n}\right]$ : By changing the order of the integration we have from (340) and (341),

$$
\begin{aligned}
\left\|\Phi_{n, \lambda}^{(1)}\left[f_{n}\right]\right\|_{L^{1}(\Omega)} \leq & 2 \pi \int_{1}^{\infty} \int_{s}^{\infty}\left|K_{\mu_{n}}(\sqrt{\lambda} r)\right| r \mathrm{~d} r\left|I_{\mu_{n}}(\sqrt{\lambda} s)\right|\left|g_{n}\right| \mathrm{d} s \\
\leq & C \int_{1}^{\max \left\{\frac{1}{\Re(\sqrt{\lambda})}, 1\right\}}\left(|\lambda|^{-1}+|\lambda|^{-\frac{\Re\left(\mu_{n}\right)+1}{2}} s^{1-\Re\left(\mu_{n}\right)}\right)|\sqrt{\lambda} s|^{\Re\left(\mu_{n}\right)}\left|g_{n}\right| \mathrm{d} s \\
& +C \int_{\max \left\{\frac{1}{\Re(\sqrt{\lambda})}, 1\right\}}^{\infty}|\lambda|^{-\frac{3}{4}} s^{\frac{1}{2}} e^{-s \Re(\sqrt{\lambda})}|\sqrt{\lambda} s|^{-\frac{1}{2}} e^{s \Re(\sqrt{\lambda})}\left|g_{n}\right| \mathrm{d} s \\
\leq & C|\lambda|^{-\frac{1}{2}}\left\|g_{n}\right\|_{L^{1}(\Omega)} .
\end{aligned}
$$

Here we have used the fact $\Re\left(\mu_{n}\right) \geq 1$ and thus $|\lambda|^{-1} s^{-1}|\sqrt{\lambda} s|^{\Re\left(\mu_{n}\right)} \leq C|\lambda|^{-\frac{1}{2}}$ if $1 \leq s \leq$ $1 / \Re(\sqrt{\lambda})$. Next we give the estimate of $L^{\infty}$ norm. Let $\Re(\sqrt{\lambda}) \leq r^{-1}$. Then Lemma A.2 and (342) imply

$$
\left|\Phi_{n, \lambda}^{(1)}\left[f_{n}\right](r)\right| \leq C|\sqrt{\lambda} r|^{-\Re\left(\mu_{n}\right)}|\lambda|^{\frac{\Re\left(\mu_{n}\right)}{2}} r^{1+\Re\left(\mu_{n}\right)}\left\|g_{n}\right\|_{L^{\infty}(\Omega)} \leq C|\lambda|^{-\frac{1}{2}}\left\|g_{n}\right\|_{L^{\infty}(\Omega)} .
$$

If $\Re(\sqrt{\lambda}) \geq r^{-1}$ then we have from Lemma A.3 and (343),

$$
\begin{aligned}
\left|\Phi_{n, \lambda}^{(1)}\left[f_{n}\right](r)\right| & \leq C|\sqrt{\lambda} r|^{-\frac{1}{2}} e^{-r \Re(\sqrt{\lambda})}\left(|\lambda|^{-\frac{1}{2}}+|\lambda|^{-\frac{3}{4}} r^{-\frac{1}{2}} e^{r \Re(\sqrt{\lambda})}\right)\left\|g_{n}\right\|_{L^{\infty}(\Omega)} \\
& \leq C|\lambda|^{-\frac{1}{2}}\left\|g_{n}\right\|_{L^{\infty}(\Omega)} .
\end{aligned}
$$


The estimates (199), (200), and (201) imply that $\Phi_{n, \lambda}^{(1)}[\cdot]$ is bounded from $L^{1}(\Omega)^{2}$ to $L^{1}(\Omega)$ and from $L^{\infty}(\Omega)^{2}$ to $L^{\infty}(\Omega)$ with the estimate $C|\lambda|^{-1 / 2}$ of its operator norm. Hence, by the interpolation theorem $\Phi_{n, \lambda}^{(1)}[\cdot]$ is bounded from $L^{p}(\Omega)^{2}$ to $L^{p}(\Omega)$ for all $1 \leq p \leq \infty$ with the same estimate.

(ii) Estimate of $\Phi_{n, \lambda}^{(2)}\left[f_{n}\right]$ : The proof is parallel to that for $\Phi_{n, \lambda}^{(1)}\left[f_{n}\right]$. Thus we omit the details.

(iii) Estimate of $\Phi_{n, \lambda}^{(3)}\left[f_{n}\right]$ : By changing the order of the integration we have from (342) and (343),

$$
\begin{aligned}
& \left\|\Phi_{n, \lambda}^{(3)}\left[f_{n}\right]\right\|_{L^{1}(\Omega)} \leq 2 \pi \int_{1}^{\infty} \int_{1}^{s}\left|I_{\mu_{n}}(\sqrt{\lambda} r)\right| r \mathrm{~d} r\left|K_{\mu_{n}}(\sqrt{\lambda} s)\right|\left|j_{n}\right| \mathrm{d} s \\
& \leq C \int_{1}^{\max \left\{\frac{1}{\Re(\sqrt{\lambda})}, 1\right\}}|\lambda|^{\frac{\Re\left(\mu_{n}\right)}{2}} s^{2+\Re\left(\mu_{n}\right)}|\sqrt{\lambda} s|^{-\Re\left(\mu_{n}\right)}\left|j_{n}\right| \mathrm{d} s \\
& \quad+C \int_{\max \left\{\frac{1}{\Re(\sqrt{\lambda})}, 1\right\}}^{\infty} s\left(|\lambda|^{-\frac{1}{2}}+|\lambda|^{-\frac{3}{4}} s^{-\frac{1}{2}} e^{s \Re(\sqrt{\lambda})}\right)|\sqrt{\lambda} s|^{-\frac{1}{2}} e^{-s \Re(\sqrt{\lambda})}\left|j_{n}\right| \mathrm{d} s \\
& \leq C|\lambda|^{-\frac{1}{2}}\left\|j_{n}\right\|_{L^{1}(\Omega)} .
\end{aligned}
$$

Let $\Re(\sqrt{\lambda}) \geq r^{-1}$. Then Lemma A.3 leads to

$$
\begin{aligned}
\left|\Phi_{n, \lambda}^{(3)}\left[f_{n}\right](r)\right| & \leq C|\sqrt{\lambda} r|^{-\frac{1}{2}} e^{r \Re(\sqrt{\lambda})} \int_{r}^{\infty}|\sqrt{\lambda} s|^{-\frac{1}{2}} e^{-s \Re(\sqrt{\lambda})} \mathrm{d} s\left\|j_{n}\right\|_{L^{\infty}(\Omega)} \\
& \leq C|\lambda|^{-\frac{1}{2}}\left\|j_{n}\right\|_{L^{\infty}(\Omega)} .
\end{aligned}
$$

On the other hand, if $\Re(\sqrt{\lambda}) \leq r^{-1}$ then Lemma A.2 and Lemma A.3 yield

$$
\begin{aligned}
&\left|\Phi_{n, \lambda}^{(3)}\left[f_{n}\right](r)\right| \leq C \mid\left.\sqrt{\lambda} r\right|^{\Re\left(\mu_{n}\right)}\left(\int_{r}^{\frac{1}{\Re(\sqrt{\lambda})}}|\sqrt{\lambda} s|^{-\Re\left(\mu_{n}\right)} \mathrm{d} s\right. \\
&\left.+\int_{\frac{1}{\Re(\sqrt{\lambda})}}^{\infty}|\sqrt{\lambda} s|^{-\frac{1}{2}} e^{-s \Re(\sqrt{\lambda})} \mathrm{d} s\right)\left\|j_{n}\right\|_{L^{\infty}(\Omega)} \\
& \leq C|\lambda|^{-\frac{1}{2}}\left\|j_{n}\right\|_{L^{\infty}(\Omega)} .
\end{aligned}
$$

As in the proof for $\Phi_{n, \lambda}^{(1)}[\cdot]$ above, the estimates (202), (203), and (204) show that $\Phi_{n, \lambda}^{(3)}[\cdot]$ is bounded from $L^{p}(\Omega)^{2}$ to $L^{p}(\Omega)$ for all $1 \leq p \leq \infty$ with the estimate $C|\lambda|^{-1 / 2}$ of its operator norm.

(iv) Estimate of $\Phi_{n, \lambda}^{(4)}\left[f_{n}\right]$ : The proof is parallel to that for $\Phi_{n, \lambda}^{(3)}\left[f_{n}\right]$. Thus we omit the details. The proof of Lemma 3.21 is complete.

Next we estimate the $L^{p}$ norm of the modified Bessel function $K_{\mu}$.

Lemma 3.22 Let $1 \leq p \leq \infty$ and let $\lambda \in \Sigma_{\pi-\epsilon}$ for some $\epsilon \in\left(0, \frac{\pi}{2}\right)$. Then there is a positive constant $C=C(\alpha, n, \epsilon, p)$ such that the following statements hold. If $\Re(\sqrt{\lambda}) \leq 1$ then

$$
\begin{aligned}
\left\|K_{\mu_{n}}(\sqrt{\lambda} \cdot)\right\|_{L^{p}(\Omega)} \leq \frac{C}{|\lambda|^{\frac{\Re\left(\mu_{n}\right)}{2}+\frac{1}{p}-\frac{1}{2}}}, & & 1 \leq p \leq 2, \\
\left\|K_{\mu_{n}}(\sqrt{\lambda} \cdot)\right\|_{L^{p}(\Omega)} \leq \frac{C}{|\lambda|^{\frac{\Re\left(\mu_{n}\right)}{2}}}, & & 2 \leq p \leq \infty .
\end{aligned}
$$


On the other hand, if $\Re(\sqrt{\lambda}) \geq 1$ then

$$
\left\|K_{\mu_{n}}(\sqrt{\lambda} \cdot)\right\|_{L^{p}(\Omega)} \leq \frac{C e^{-\Re(\sqrt{\lambda})}}{|\lambda|^{\frac{1}{4}+\frac{1}{2 p}}}, \quad 1 \leq p \leq \infty .
$$

Proof. Let $\Re(\sqrt{\lambda}) \leq 1$ and $1 \leq p<\infty$. Then Lemma A.2 and Lemma A.3 imply

$$
\begin{aligned}
\left\|K_{\mu_{n}}(\sqrt{\lambda} \cdot)\right\|_{L^{p}(\Omega)}^{p} & \leq C^{p} \int_{1}^{\frac{1}{\Re(\sqrt{\lambda})}}|\sqrt{\lambda} r|^{-p \Re\left(\mu_{n}\right)} r \mathrm{~d} r+C^{p} \int_{\frac{1}{\Re(\sqrt{\lambda})}}^{\infty}|\sqrt{\lambda} r|^{-\frac{p}{2}} e^{-p r \Re(\sqrt{\lambda})} r \mathrm{~d} r \\
& \leq C^{p}|\lambda|^{-\frac{p \Re\left(\mu_{n}\right)}{2}} \int_{1}^{\frac{1}{\Re(\sqrt{\lambda})}} r^{1-p \Re\left(\mu_{n}\right)} \mathrm{d} r+\frac{C^{p}}{p|\lambda|}
\end{aligned}
$$

Thus, using $\Re\left(\mu_{n}\right)>|n| \geq 1$ and $\Re(\sqrt{\lambda}) \leq 1$, we obtain (205) and (206). The estimate for the case $p=\infty$ follows in the similar manner. Next we consider the case $\Re(\sqrt{\lambda}) \geq 1$. Then Lemma A.3 yields for $1 \leq p<\infty$,

$$
\begin{aligned}
\left\|K_{\mu_{n}}(\sqrt{\lambda} \cdot)\right\|_{L^{p}(\Omega)}^{p} \leq C^{p} \int_{1}^{\infty}|\sqrt{\lambda} r|^{-\frac{p}{2}} e^{-p r \Re(\sqrt{\lambda})} r \mathrm{~d} r & \leq \frac{C^{p}}{|\lambda|^{\frac{p}{4}}} \int_{1}^{\infty} r^{1-\frac{p}{2}} e^{-p r \Re(\sqrt{\lambda})} \mathrm{d} r \\
& \leq \frac{C^{p} e^{-p \Re(\sqrt{\lambda})}}{|\lambda|^{\frac{p}{4}+\frac{1}{2}}}
\end{aligned}
$$

The estimate for the case $p=\infty$ directly follows from Lemma A.3. The proof is complete.

We are now in position to prove the estimates for the vorticity $\operatorname{rot}\left(\lambda+\left.A_{\alpha}\right|_{\mathcal{P}_{n} L_{\sigma}^{2}}\right)^{-1} f_{n}$, and hence, for the derivative $\nabla\left(\lambda+\left.A_{\alpha}\right|_{\mathcal{P}_{n} L_{\sigma}^{2}}\right)^{-1} f_{n}$.

Theorem 3.23 Let $1 \leq q \leq \infty$ and let $\lambda \in \rho\left(-\left.A_{\alpha}\right|_{\mathcal{P}_{n} L_{\sigma}^{2}}\right) \cap \Sigma_{\pi-\epsilon}$ for some $\epsilon \in\left(0, \frac{\pi}{2}\right)$. Then there is a positive constant $C=C(\alpha, n, \epsilon, q)$ such that the following statements hold. Let $f_{n} \in \mathcal{P}_{n} C_{0, \sigma}^{\infty}(\Omega)$.

(1) If $\Re(\sqrt{\lambda}) \leq 1$ and $1 \leq q \leq 2$ then

$$
\left\|\operatorname{rot}\left(\lambda+\left.A_{\alpha}\right|_{\mathcal{P}_{n} L_{\sigma}^{2}}\right)^{-1} f_{n}\right\|_{L^{q}(\Omega)} \leq \frac{C}{|\lambda|^{\frac{1}{2}}}\left(\frac{|\lambda|^{-\frac{\Re\left(\mu_{n}\right)}{2}}}{\left|F_{n}(\sqrt{\lambda} ; \alpha)\right|}+1\right)\left\|f_{n}\right\|_{L^{q}(\Omega)} .
$$

(2) If $\Re(\sqrt{\lambda}) \leq 1$ and $2 \leq q \leq \infty$ then

$$
\left\|\operatorname{rot}\left(\lambda+\left.A_{\alpha}\right|_{\mathcal{P}_{n} L_{\sigma}^{2}}\right)^{-1} f_{n}\right\|_{L^{q}(\Omega)} \leq \frac{C}{|\lambda|^{1-\frac{1}{q}}}\left(\frac{|\lambda|^{-\frac{\Re\left(\mu_{n}\right)}{2}}}{\left|F_{n}(\sqrt{\lambda} ; \alpha)\right|}+1\right)\left\|f_{n}\right\|_{L^{q}(\Omega)} .
$$

(3) If $\Re(\sqrt{\lambda}) \geq 1$ and $1 \leq q \leq \infty$ then

$$
\left\|\operatorname{rot}\left(\lambda+\left.A_{\alpha}\right|_{\mathcal{P}_{n} L_{\sigma}^{2}}\right)^{-1} f_{n}\right\|_{L^{q}(\Omega)} \leq \frac{C}{|\lambda|^{\frac{1}{2}}}\left(\frac{|\lambda|^{-\frac{3}{4}} e^{-\Re(\sqrt{\lambda})}}{\left|F_{n}(\sqrt{\lambda} ; \alpha)\right|}+1\right)\left\|f_{n}\right\|_{L^{q}(\Omega)} .
$$

Moreover, except for the end point cases $q=1, \infty$ the above statements (1), (2), and (3) hold also for $\left\|\nabla\left(\lambda+\left.A_{\alpha}\right|_{\mathcal{P}_{n} L_{\sigma}^{2}}\right)^{-1} f_{n}\right\|_{L^{q}(\Omega)}$. 
Proof. We recall that (79) and (35) imply

$$
\operatorname{rot}\left(\lambda+\left.A_{\alpha}\right|_{\mathcal{P}_{n} L_{\sigma}^{2}}\right)^{-1} f_{n}=-\frac{c_{n, \lambda}\left[f_{n}\right]}{F_{n}(\sqrt{\lambda} ; \alpha)} K_{\mu_{n}}(\sqrt{\lambda} r) e^{i n \theta}+\Phi_{n, \lambda}\left[f_{n}\right](r) e^{i n \theta}
$$

in the polar coordinates. Hence, (210), (211), and (212) follow from Proposition 3.16, Lemma 3.21, and Lemma 3.22. Then the estimate of $\left\|\nabla\left(\lambda+\left.A_{\alpha}\right|_{\mathcal{P}_{n} L_{\sigma}^{2}}\right)^{-1} f_{n}\right\|_{L^{q}(\Omega)}$ for $1<q<\infty$ is derived from the inequality

$$
\|\nabla h\|_{L^{q}(\Omega)} \leq C\|\operatorname{rot} h\|_{L^{q}(\Omega)} \quad \text { for } h \in W_{0}^{1, q}(\Omega)^{2} \cap L_{\sigma}^{q}(\Omega) .
$$

The proof is complete.

\subsection{Resolvent estimates in $\mathcal{Q}_{n} L_{\sigma}^{2}(\Omega)$ and $\mathcal{P}_{ \pm 1} L_{\sigma}^{2}(\Omega)$ by energy method}

The resolvent estimates in Theorems 3.19 and 3.23 highly depend on the Fourier mode $n$. To overcome this difficulty we use the energy method for the resolvent estimates in $\mathcal{Q}_{n} L_{\sigma}^{2}(\Omega)$ in Section 3.4.1. The key observation here is that the Hardy-type inequality does hold in $\mathcal{Q}_{n} L_{\sigma}^{2}(\Omega)$ as long as $|n| \geq 1$, which follows from Lemma 2.2. In Section 3.4.2 we also apply the energy argument to the resolvent problem in $\mathcal{P}_{ \pm 1} L_{\sigma}^{2}(\Omega)$, which is used to investigate the zero points of $F_{n}(\sqrt{\lambda} ; \alpha)$ for $|n|=1$ and $|\alpha| \ll 1$ later in Section 3.6.

\subsubsection{Estimates in $\mathcal{Q}_{n} L_{\sigma}^{2}(\Omega)$ for $n \geq 1$ by energy method}

The main result of this section is the following theorem.

Theorem 3.24 Let $n$ be any positive integer satisfying $|\alpha|<n$. Then the following statements hold.

(1) The spectrum $\sigma\left(-\left.A_{\alpha}\right|_{\mathcal{Q}_{n} L_{\sigma}^{2}}\right)$ is included in the set

$$
\left\{z \in \mathbb{C}|| \Im(z) \mid \leq-\frac{|\alpha|}{n-|\alpha|} \Re(z)\right\} .
$$

(2) The perturbed Stokes semigroup $\left\{e^{-t A_{\alpha}}\right\}_{t \geq 0}$ in $\mathcal{Q}_{n} L_{\sigma}^{2}(\Omega)$ satisfies the estimates

$$
\begin{aligned}
& \left\|e^{-t A_{\alpha}} f\right\|_{L^{2}(\Omega)} \leq\|f\|_{L^{2}(\Omega)}, \quad t>0, \quad f \in \mathcal{Q}_{n} L_{\sigma}^{2}(\Omega), \\
& \left\|\nabla e^{-t A_{\alpha}} f\right\|_{L^{2}(\Omega)} \leq C t^{-\frac{1}{2}}\|f\|_{L^{2}(\Omega)}, \quad t>0, \quad f \in \mathcal{Q}_{n} L_{\sigma}^{2}(\Omega) \text {. }
\end{aligned}
$$

Here the constant $C$ depends only on $\alpha$ and $n$. Moreover, for $1<q \leq 2 \leq p<\infty$ it follows that

$$
\left\|e^{-t A_{\alpha}} f\right\|_{L^{p}(\Omega)} \leq C t^{-\frac{1}{q}+\frac{1}{p}}\|f\|_{L^{q}(\Omega)}, \quad t>0, \quad f \in \mathcal{Q}_{n} L_{\sigma}^{2}(\Omega) \cap L^{q}(\Omega)^{2},
$$

where the constant $C$ depends only on $\alpha, n$, $p$, and $q$.

Remark 3.25 By the density argument we have from (216) and (218),

$$
\lim _{t \rightarrow \infty}\left\|e^{-t A_{\alpha}} f\right\|_{L^{2}(\Omega)}=0 \quad \text { for all } f \in \mathcal{Q}_{n} L_{\sigma}^{2}(\Omega) .
$$


Proof of Theorem 3.24. To prove (215) let us compute the numerical range of $-\left.A_{\alpha}\right|_{\mathcal{Q}_{n} L_{\sigma}^{2}}$, denoted by $W\left(-\left.A_{\alpha}\right|_{\mathcal{Q}_{n} L_{\sigma}^{2}}\right)$ :

$$
\begin{aligned}
& W\left(-\left.A_{\alpha}\right|_{\mathcal{Q}_{n} L_{\sigma}^{2}}\right) \\
& =\left\{\left\langle-A_{\alpha} v, v\right\rangle_{L^{2}(\Omega)} \mid v \in D_{L_{\sigma}^{2}}\left(A_{\alpha}\right) \cap \mathcal{Q}_{n} L_{\sigma}^{2}(\Omega),\|v\|_{L^{2}(\Omega)}=1\right\} .
\end{aligned}
$$

From $-A_{\alpha} v=\mathbb{P} \Delta v-\alpha \mathbb{P}\left(U^{\perp} \operatorname{rot} v\right)$ we have

$$
\left\langle-A_{\alpha} v, v\right\rangle_{L^{2}(\Omega)}=-\|\nabla v\|_{L^{2}(\Omega)}^{2}-\alpha\left\langle U^{\perp} \operatorname{rot} v, v\right\rangle_{L^{2}(\Omega)},
$$

and hence, the Schwartz inequality and $|U(x)| \leq|x|^{-1}$ imply

$$
\left|\Im\left\langle-A_{\alpha} v, v\right\rangle_{L^{2}(\Omega)}\right|=|\alpha|\left|\Im\left\langle U^{\perp} \operatorname{rot} v, v\right\rangle_{L^{2}(\Omega)}\right| \leq|\alpha|\|\operatorname{rot} v\|_{L^{2}(\Omega)}\left\||x|^{-1} v\right\|_{L^{2}(\Omega)} .
$$

Since $v \in W_{0}^{1,2}(\Omega)^{2} \cap L_{\sigma}^{2}(\Omega)$ we have $\|\operatorname{rot} v\|_{L^{2}(\Omega)}=\|\nabla v\|_{L^{2}(\Omega)}$, while from the fact the function $|x|^{-1}$ is radially symmetric the velocity $v \in \mathcal{Q}_{n} L_{\sigma}^{2}(\Omega)$ satisfies

$$
\begin{aligned}
\left\|\left.x\right|^{-1} v\right\|_{L^{2}(\Omega)}^{2}=\sum_{|l| \geq n+1}\left\||x|^{-1} \mathcal{P}_{l} v\right\|_{L^{2}(\Omega)}^{2} & =\sum_{|l| \geq n+1} \int_{0}^{2 \pi} \int_{1}^{\infty} \frac{1}{r^{2}}\left(\left|v_{r, l}\right|^{2}+\left|v_{\theta, l}\right|^{2}\right) r \mathrm{~d} r \mathrm{~d} \theta \\
& \leq \sum_{|l| \geq n+1} \frac{1}{(|l|-1)^{2}}\left\|\nabla \mathcal{P}_{l} v\right\|_{L^{2}(\Omega)}^{2} .
\end{aligned}
$$

Here we have used Lemma 2.2 in the last line. Then the identity (28) yields

$$
\left\|\left.x\right|^{-1} v\right\|_{L^{2}(\Omega)}^{2} \leq \frac{1}{n^{2}}\|\nabla v\|_{L^{2}(\Omega)}^{2} .
$$

Collecting (222) and (223), we have

$$
\left|\Im\left\langle-A_{\alpha} v, v\right\rangle_{L^{2}(\Omega)}\right| \leq \frac{|\alpha|}{n}\|\nabla v\|_{L^{2}(\Omega)}^{2}, \quad v \in D_{L_{\sigma}^{2}}\left(A_{\alpha}\right) \cap \mathcal{Q}_{n} L_{\sigma}^{2}(\Omega), \quad n \geq 1 .
$$

The similar calculation yields

$$
\begin{aligned}
\Re\left\langle-A_{\alpha} v, v\right\rangle_{L^{2}(\Omega)} & =-\|\nabla v\|_{L^{2}(\Omega)}^{2}-\alpha \Re\left\langle U^{\perp} \operatorname{rot} v, v\right\rangle_{L^{2}(\Omega)} \\
& \leq-\left(1-\frac{|\alpha|}{n}\right)\|\nabla v\|_{L^{2}(\Omega)}^{2}, \quad v \in D_{L_{\sigma}^{2}}\left(A_{\alpha}\right) \cap \mathcal{Q}_{n} L_{\sigma}^{2}(\Omega), \quad n \geq 1 .
\end{aligned}
$$

Combining (224) and (225), we obtain for $|\alpha|<n$,

$$
\begin{aligned}
\left|\Im\left\langle-A_{\alpha} v, v\right\rangle_{L^{2}(\Omega)}\right| & \leq \frac{|\alpha|}{n}\left(-\frac{n}{n-|\alpha|} \Re\left\langle-A_{\alpha} v, v\right\rangle_{L^{2}(\Omega)}\right) \\
& =-\frac{|\alpha|}{n-|\alpha|} \Re\left\langle-A_{\alpha} v, v\right\rangle_{L^{2}(\Omega)},
\end{aligned}
$$

which gives the estimate of the numerical range of $-\left.A_{\alpha}\right|_{\mathcal{Q}_{n} L_{\sigma}^{2}}$. Since we have already known the sectoriality of $-A_{\alpha}$, the spectrum $\sigma\left(-\left.A_{\alpha}\right|_{\mathcal{Q}_{n} L_{\sigma}^{2}}\right)$ is included in the set given in (215) by [27, V-3, Theorem 3.2], and the estimate (225) also implies that $\left\{e^{-t A_{\alpha}}\right\}_{t \geq 0}$ is a 
contraction semigroup in $\mathcal{Q}_{n} L_{\sigma}^{2}(\Omega)$ when $|\alpha|<n$. To derive the derivative estimates for the semigroup let us consider the resolvent problem for $\lambda \in \rho\left(-\left.A_{\alpha}\right|_{\mathcal{Q}_{n} L_{\sigma}^{2}}\right)$ :

$$
\lambda v+A_{\alpha} v=f, \quad v \in D_{L_{\sigma}^{2}}\left(A_{\alpha}\right) \cap \mathcal{Q}_{n} L_{\sigma}^{2}(\Omega), \quad f \in \mathcal{Q}_{n} L_{\sigma}^{2}(\Omega) .
$$

From (224) and (225) we have

$$
\begin{aligned}
& |\Im(\lambda)|\|v\|_{L^{2}(\Omega)}^{2} \leq \frac{|\alpha|}{n}\|\nabla v\|_{L^{2}(\Omega)}^{2}+\|f\|_{L^{2}(\Omega)}\|v\|_{L^{2}(\Omega)}, \\
& \Re(\lambda)\|v\|_{L^{2}(\Omega)}^{2}+\left(1-\frac{|\alpha|}{n}\right)\|\nabla v\|_{L^{2}(\Omega)}^{2} \leq\|f\|_{L^{2}(\Omega)}\|v\|_{L^{2}(\Omega)} .
\end{aligned}
$$

In particular, we have for $|\alpha|<n$,

$$
\left(|\Im(\lambda)|+\frac{|\alpha|}{n-|\alpha|} \Re(\lambda)\right)\|v\|_{L^{2}(\Omega)}^{2} \leq \frac{n}{n-|\alpha|}\|f\|_{L^{2}(\Omega)}\|v\|_{L^{2}(\Omega)},
$$

which implies

$$
\left\|\left(\lambda+\left.A_{\alpha}\right|_{\mathcal{Q}_{n} L_{\sigma}^{2}}\right)^{-1} f\right\|_{L^{2}(\Omega)} \leq \frac{1}{|\Im(\lambda)|+\frac{|\alpha|}{n-|\alpha|} \Re(\lambda)} \frac{n}{n-|\alpha|}\|f\|_{L^{2}(\Omega)},
$$

when $\lambda$ belongs to the sector $\left\{z \in \mathbb{C}|| \Im(z) \mid>-\frac{|\alpha|}{n-|\alpha|} \Re(z)\right\}$. Moreover, in this sector we have from (229) and (230),

$$
\begin{aligned}
& \left(1-\frac{|\alpha|}{n}\right)\left\|\nabla\left(\lambda+\left.A_{\alpha}\right|_{\mathcal{Q}_{n} L_{\sigma}^{2}}\right)^{-1} f\right\|_{L^{2}(\Omega)}^{2} \\
& \leq \frac{1}{|\Im(\lambda)|+\frac{|\alpha|}{n-|\alpha|} \Re(\lambda)} \frac{n}{n-|\alpha|}\|f\|_{L^{2}(\Omega)}^{2} \\
& \quad+\max \{-\Re(\lambda), 0\}\left(\frac{1}{|\Im(\lambda)|+\frac{|\alpha|}{n-|\alpha|} \Re(\lambda)} \frac{n}{n-|\alpha|}\right)^{2}\|f\|_{L^{2}(\Omega)}^{2} .
\end{aligned}
$$

Now let us recall the representation of $\left.e^{-t A_{\alpha}}\right|_{\mathcal{Q}_{n} L_{\sigma}^{2}}$ in the Dunford integral

$$
\left.e^{-t A_{\alpha}}\right|_{\mathcal{Q}_{n} L_{\sigma}^{2}}=\frac{1}{2 \pi i} \int_{\gamma} e^{t \lambda}\left(\lambda+\left.A_{\alpha}\right|_{\mathcal{Q}_{n} L_{\sigma}^{2}}\right)^{-1} \mathrm{~d} \lambda, \quad t>0
$$

Here $\gamma=\gamma_{\kappa}$ is the curve $\{\lambda \in \mathbb{C}|| \arg \lambda|=\eta,| \lambda \mid \geq \kappa\} \cup\{\lambda \in \mathbb{C}|| \arg \lambda|\leq \eta,| \lambda \mid=\kappa\}$ for some $\eta \in\left(\frac{\pi}{2}, \pi\right)$ and $0<\kappa \ll 1$, oriented counterclockwise. We take $\eta$ close to $\frac{\pi}{2}$ so that $\gamma$ belongs to the sector $\left\{z \in \mathbb{C}|| \Im(z) \mid>-\frac{|\alpha|}{n-|\alpha|} \Re(z)\right\}$, and we denote by $\gamma_{0}$ the limit curve obtained by taking $\kappa=0$. Then for $f \in \mathcal{Q}_{n} L_{\sigma}^{2}(\Omega)$ we have from (231) and by taking $\kappa \rightarrow 0$,

$$
\begin{aligned}
\left\|\nabla e^{-t A_{\alpha}} f\right\|_{L^{2}(\Omega)} & \leq \frac{1}{2 \pi} \int_{\gamma_{0}}\left\|e^{t \lambda} \nabla\left(\lambda+A_{\alpha}\right)^{-1} f\right\|_{L^{2}(\Omega)}|\mathrm{d} \lambda| \\
& \leq C \int_{0}^{\infty} r^{-\frac{1}{2}} e^{-c t r} \mathrm{~d} r\|f\|_{L^{2}(\Omega)} \leq C t^{-\frac{1}{2}}\|f\|_{L^{2}(\Omega)},
\end{aligned}
$$

which proves (217). Finally we will show (218). To this end let us consider the adjoint operator $\left.A_{\alpha}\right|_{\mathcal{Q}_{n} L_{\sigma}^{2}} ^{*}$ of $\left.A_{\alpha}\right|_{\mathcal{Q}_{n} L_{\sigma}^{2}}$ in $\mathcal{Q}_{n} L_{\sigma}^{2}(\Omega)$. It is easy to see that $\left.A_{\alpha}\right|_{\mathcal{Q}_{n} L_{\sigma}^{2}} ^{*}=\left.A_{\alpha}^{*}\right|_{\mathcal{Q}_{n} L_{\sigma}^{2}}$ 
holds, and since $A_{\alpha} v=A v+\alpha \mathbb{P}(U \cdot \nabla v+v \cdot \nabla U)$ we have a representation of $\left.A_{\alpha}\right|_{\mathcal{Q}_{n} L_{\sigma}^{2}} ^{*}$ in $\mathcal{Q}_{n} L_{\sigma}^{2}(\Omega)$ such as

$$
\begin{aligned}
D\left(\left.A_{\alpha}\right|_{\mathcal{Q}_{n} L_{\sigma}^{2}} ^{*}\right) & =W^{2,2}(\Omega)^{2} \cap W_{0}^{1,2}(\Omega)^{2} \cap \mathcal{Q}_{n} L_{\sigma}^{2}(\Omega), \\
\left.A_{\alpha}\right|_{\mathcal{Q}_{n} L_{\sigma}^{2}} ^{*} u & =A u-\alpha \mathbb{P}\left(U \cdot \nabla u+\sum_{j=1,2} U_{j} \nabla u_{j}\right), \quad u \in D\left(\left.A_{\alpha}\right|_{\mathcal{Q}_{n} L_{\sigma}^{2}} ^{*}\right) .
\end{aligned}
$$

As in the derivations of (225), we have

$$
\begin{aligned}
\Re\left\langle-A_{\alpha}^{*} u, u\right\rangle_{L^{2}(\Omega)} & =-\|\nabla u\|_{L^{2}(\Omega)}^{2}+\alpha \Re\left\langle\sum_{j=1,2} U_{j} \nabla u_{j}, u\right\rangle_{L^{2}(\Omega)} \\
& \leq-\|\nabla u\|_{L^{2}(\Omega)}^{2}+|\alpha|\|\nabla u\|_{L^{2}(\Omega)}\left\||x|^{-1} u\right\|_{L^{2}(\Omega)} \\
& \leq-\left(1-\frac{|\alpha|}{n}\right)\|\nabla u\|_{L^{2}(\Omega)}^{2} .
\end{aligned}
$$

Here we have used (223) in the last line. Let $u \in D\left(\left.A_{\alpha}\right|_{\mathcal{Q}_{n} L_{\sigma}^{2}} ^{*}\right)$ be the solution to the resolvent equation

$$
\lambda u+A_{\alpha}^{*} u=g, \quad g \in \mathcal{Q}_{n} L_{\sigma}^{2}(\Omega),
$$

for $\lambda \in\left\{z \in \mathbb{C}|| \Im(z) \mid>-\frac{|\alpha|}{n-|\alpha|} \Re(z)\right\}$. Since $\bar{\lambda}$ belongs to $\rho\left(-\left.A_{\alpha}\right|_{\mathcal{Q}_{n} L_{\sigma}^{2}}\right)$ we already know $\lambda \in \rho\left(-\left.A_{\alpha}^{*}\right|_{\mathcal{Q}_{n} L_{\sigma}^{2}}\right)$. Moreover, the estimate (230) implies that there is $\epsilon_{0}=$ $\epsilon_{0}(n, \alpha) \in(0, \pi / 2)$ such that $\Sigma_{\pi-\epsilon_{0}} \subset\left\{z \in \mathbb{C}|| \Im(z) \mid>-\frac{|\alpha|}{n-|\alpha|} \Re(z)\right\}$ and

$$
\|u\|_{L^{2}(\Omega)}=\left\|\left(\lambda+A_{\alpha}^{*}\right)^{-1} g\right\|_{L^{2}(\Omega)} \leq \frac{C}{|\lambda|}\|g\|_{L^{2}(\Omega)}, \quad \lambda \in \Sigma_{\pi-\epsilon_{0}} .
$$

Here the constant $C$ depends only on $\alpha$ and $n$. Hence (235) and (237) yield

$$
\begin{aligned}
\left(1-\frac{|\alpha|}{n}\right)\|\nabla u\|_{L^{2}(\Omega)}^{2} \leq \Re\left\langle A_{\alpha}^{*} u, u\right\rangle_{L^{2}(\Omega)} & =-\Re(\lambda)\|u\|_{L^{2}(\Omega)}^{2}+\Re\langle g, u\rangle_{L^{2}(\Omega)} \\
& \leq \frac{C}{|\lambda|}\|g\|_{L^{2}(\Omega)}^{2}, \quad \lambda \in \Sigma_{\pi-\epsilon_{0}} .
\end{aligned}
$$

Now we have proved the estimate

$$
\left\|\nabla\left(\lambda+\left.A_{\alpha}\right|_{\mathcal{Q}_{n} L_{\sigma}^{2}} ^{*}\right)^{-1} g\right\|_{L^{2}(\Omega)} \leq \frac{C}{|\lambda|^{\frac{1}{2}}}\|g\|_{L^{2}(\Omega)}, \quad \lambda \in \Sigma_{\pi-\epsilon_{0}} .
$$

Let us back to the estimate of $\left(\lambda+\left.A_{\alpha}\right|_{\mathcal{Q}_{n} L_{\sigma}^{2}}\right)^{-1}$. Let $\lambda \in \Sigma_{\pi-\epsilon_{0}}$. Let $1<q<2$ and $\frac{1}{q}+\frac{1}{q^{\prime}}=1$. Then we have for any $f \in \mathcal{Q}_{n} L_{\sigma}^{2}(\Omega) \cap L^{q}(\Omega)^{2}$ and $g \in \mathcal{Q}_{n} L_{\sigma}^{2}(\Omega)$,

$$
\begin{aligned}
\left|\left\langle\left(\lambda+A_{\alpha}\right)^{-1} f, g\right\rangle_{L^{2}(\Omega)}\right| & =\left|\left\langle f,\left(\bar{\lambda}+A_{\alpha}^{*}\right)^{-1} g\right\rangle_{L^{2}(\Omega)}\right| \\
& \leq\|f\|_{L^{q}(\Omega)}\left\|\left(\bar{\lambda}+A_{\alpha}^{*}\right)^{-1} g\right\|_{L^{q^{\prime}}(\Omega)} \\
& \leq C\|f\|_{L^{q}(\Omega)}\left\|\nabla\left(\bar{\lambda}+A_{\alpha}^{*}\right)^{-1} g\right\|_{L^{2}(\Omega)}^{1-\frac{2}{q^{\prime}}}\left\|\left(\bar{\lambda}+A_{\alpha}^{*}\right)^{-1} g\right\|_{L^{2}(\Omega)}^{\frac{2}{q^{\prime}}} \\
& \leq \frac{C}{|\lambda|^{\frac{3}{2}-\frac{1}{q}}}\|f\|_{L^{q}(\Omega)}\|g\|_{L^{2}(\Omega)}
\end{aligned}
$$


Here we have use the Hölder inequality in the second line, the Gagliardo-Nirenberg inequality in the third line, and (237) and (238) in the last line. By the duality the estimate (238) implies that

$$
\left\|\left(\lambda+A_{\alpha}\right)^{-1} f\right\|_{L^{2}(\Omega)} \leq \frac{C}{|\lambda|^{\frac{3}{2}-\frac{1}{q}}}\|f\|_{L^{q}(\Omega)}, \quad \lambda \in \Sigma_{\pi-\epsilon_{0}}
$$

for $f \in \mathcal{Q}_{n} L_{\sigma}^{2}(\Omega) \cap L^{q}(\Omega)^{2}$. Therefore, as in the calculation in (233), we have

$$
\left\|e^{-t A_{\alpha}} f\right\|_{L^{2}(\Omega)} \leq C \int_{0}^{\infty} r^{-\frac{3}{2}+\frac{1}{q}} e^{-c t r} \mathrm{~d} r\|f\|_{L^{q}(\Omega)} \leq C t^{-\frac{1}{q}+\frac{1}{2}}\|f\|_{L^{q}(\Omega)}
$$

for all $f \in \mathcal{Q}_{n} L_{\sigma}^{2}(\Omega) \cap L^{q}(\Omega)^{2}$. Here the constant $C$ depends only on $\alpha$, $n$, and $q$. Then for $1<q<2 \leq p<\infty$ and $f \in \mathcal{Q}_{n} L_{\sigma}^{2}(\Omega) \cap L^{q}(\Omega)^{2}$ we have from the semigroup property,

$$
\begin{aligned}
\left\|e^{-t A_{\alpha}} f\right\|_{L^{p}(\Omega)} \leq C\left\|\nabla e^{-t A_{\alpha}} f\right\|_{L^{2}(\Omega)}^{1-\frac{2}{p}}\left\|e^{-t A_{\alpha}} f\right\|_{L^{2}(\Omega)}^{\frac{2}{p}} & \leq C t^{-\frac{1}{2}\left(1-\frac{2}{p}\right)}\left\|e^{-\frac{t}{2} A_{\alpha}} f\right\|_{L^{2}(\Omega)} \\
& \leq C t^{-\frac{1}{q}+\frac{1}{p}}\|f\|_{L^{q}(\Omega)}
\end{aligned}
$$

where we have also used the Gagliardo-Nirenberg inequality, (233), and (241). The proof is complete.

\subsubsection{Estimates in $\mathcal{P}_{n} L_{\sigma}^{2}(\Omega)$ for $|n|=1$ by energy method}

For $T>0$ set

$$
\beta(T)=\int_{0}^{T} \frac{1}{\tau} e^{-\frac{1}{\tau}} \mathrm{d} \tau
$$

Note that there are positive constants $C_{1}$ and $C_{2}$ such that

$$
C_{1} \log T \leq \beta(T) \leq C_{2} \log T, \quad \text { for all } T \geq e .
$$

Lemma 3.26 Let $|n|=1$ and let $v \in W_{0}^{1,2}(\Omega)^{2} \cap \mathcal{P}_{n} L_{\sigma}^{2}(\Omega)$. For any $T>0$ it follows that

$$
\left|\left\langle U^{\perp} \operatorname{rot} v, v\right\rangle_{L^{2}(\Omega)}\right| \leq \frac{1}{T}\|\nabla v\|_{L^{2}(\Omega)}\|v\|_{L^{2}(\Omega)}+\beta(T)\|\nabla v\|_{L^{2}(\Omega)}^{2} .
$$

Proof. We firstly assume that $v \in \mathcal{P}_{n} C_{0, \sigma}^{\infty}(\Omega)$ with $|n|=1$ and then extend $v$ by zero to the whole space $\mathbb{R}^{2}$. Let $v=v_{r, n} e^{i n \theta} \mathbf{e}_{r}+v_{\theta, n} e^{i n \theta} \mathbf{e}_{\theta}$ and $\operatorname{rot} v=w_{n} e^{i n \theta}$ in the polar coordinates. From the identity $\left|U^{\perp} \cdot v\right|=r^{-1}\left|v_{r, n}\right|$ in the polar coordinates we have

$$
\left|\left\langle U^{\perp} \operatorname{rot} v, v\right\rangle_{L^{2}(\Omega)}\right| \leq \int_{0}^{2 \pi} \int_{1}^{\infty} \frac{1}{r}\left|w_{n}(r)\right|\left|v_{r, n}(r)\right| r \mathrm{~d} r \mathrm{~d} \theta
$$

Then for any $T>0$ we see $\frac{1}{r}=\frac{1}{r}\left(1-e^{-\frac{r}{T}}\right)+\frac{1}{r} e^{-\frac{r}{T}} \leq \frac{1}{T}+\frac{1}{r} e^{-\frac{r}{T}}$, which gives

$$
\begin{aligned}
\left|\left\langle U^{\perp} \operatorname{rot} v, v\right\rangle_{L^{2}(\Omega)}\right| \leq & \frac{1}{T}\|\operatorname{rot} v\|_{L^{2}(\Omega)}\|v\|_{L^{2}(\Omega)} \\
& +\|\operatorname{rot} v\|_{L^{2}(\Omega)}\left(\int_{0}^{2 \pi} \int_{1}^{\infty}\left|\frac{1}{r} e^{-\frac{r}{T}} v_{r, n}(r)\right|^{2} r \mathrm{~d} r \mathrm{~d} \theta\right)^{\frac{1}{2}} .
\end{aligned}
$$


By applying the mean-value theorem we have

$$
\left|\frac{1}{r} e^{-\frac{r}{T}} v_{r, n}(r)\right| \leq \int_{0}^{1} e^{-\frac{r}{T}}\left|\left(v_{r, n}\right)^{\prime}(\sigma r)\right| \mathrm{d} \sigma,
$$

and hence the Minkowskii inequality and the condition $\left(v_{r, n}\right)^{\prime}(r)=0$ for $0 \leq r \leq 1$ imply

$$
\begin{aligned}
\left(\int_{0}^{2 \pi} \int_{1}^{\infty}\left|\frac{1}{r} e^{-\frac{r}{T}} v_{r, n}(r)\right|^{2} r \mathrm{~d} r \mathrm{~d} \theta\right)^{\frac{1}{2}} & \leq \int_{0}^{1}\left(\int_{0}^{2 \pi} \int_{1}^{\infty} e^{-\frac{2 r}{T}}\left|\left(v_{r, n}\right)^{\prime}(\sigma r)\right|^{2} r \mathrm{~d} r \mathrm{~d} \theta\right)^{\frac{1}{2}} \mathrm{~d} \sigma \\
& =\int_{0}^{1} \frac{1}{\sigma}\left(\int_{0}^{2 \pi} \int_{1}^{\infty} e^{-\frac{2 \tau}{T \sigma}}\left|\left(v_{r, n}\right)^{\prime}(\tau)\right|^{2} \tau \mathrm{d} \tau \mathrm{d} \theta\right)^{\frac{1}{2}} \mathrm{~d} \sigma \\
& \leq \int_{0}^{1} \frac{1}{\sigma} e^{-\frac{1}{T \sigma}} \mathrm{d} \sigma\left(\int_{0}^{2 \pi} \int_{1}^{\infty}\left|\left(v_{r, n}\right)^{\prime}(\tau)\right|^{2} \tau \mathrm{d} \tau \mathrm{d} \theta\right)^{\frac{1}{2}} \\
& \leq \beta(T)\|\nabla v\|_{L^{2}(\Omega)} .
\end{aligned}
$$

Here we have used Lemma 2.2 in the last line. Thus, the inequality (245) follows for any $v \in \mathcal{P}_{n} C_{0, \sigma}^{\infty}(\Omega)$ by the identity $\|\nabla v\|_{L^{2}(\Omega)}=\|\operatorname{rot} v\|_{L^{2}(\Omega)}$, and then for general $v \in$ $W_{0}^{1,2}(\Omega)^{2} \cap \mathcal{P}_{n} L_{\sigma}^{2}(\Omega)$ by the density argument. The proof is complete.

Theorem 3.27 Let $|n|=1$. Fix any $T>0$ and let $|\alpha| \beta(T)<1$. Then the spectrum $\sigma\left(-\left.A_{\alpha}\right|_{\mathcal{P}_{n} L_{\sigma}^{2}}\right)$ is included in the set

$$
\left\{z \in \mathbb{C}|| \Im(z) \mid \leq-\frac{3|\alpha| \beta(T)}{1-|\alpha| \beta(T)} \Re(z)+\frac{3|\alpha|^{3} \beta(T)}{2 T^{2}(1-|\alpha| \beta(T))^{2}}+\frac{\alpha^{2}}{2 T^{2} \beta(T)}\right\} .
$$

Proof. Let us estimate the numerical range

$$
W\left(-\left.A_{\alpha}\right|_{\mathcal{P}_{n} L_{\sigma}^{2}}\right)=\left\{\left\langle-A_{\alpha} v, v\right\rangle_{L^{2}(\Omega)} \mid v \in D_{L_{\sigma}^{2}}\left(A_{\alpha}\right) \cap \mathcal{P}_{n} L_{\sigma}^{2}(\Omega),\|v\|_{L^{2}(\Omega)}=1\right\} .
$$

By using Lemma 3.26 we have from $\|v\|_{L^{2}(\Omega)}=1$,

$$
\begin{aligned}
\left|\Im\left\langle-A_{\alpha} v, v\right\rangle_{L^{2}(\Omega)}\right| & =\left|\alpha \Im\left\langle U^{\perp} \operatorname{rot} v, v\right\rangle_{L^{2}(\Omega)}\right| \\
& \leq \frac{|\alpha|}{T}\|\nabla v\|_{L^{2}(\Omega)}+|\alpha| \beta(T)\|\nabla v\|_{L^{2}(\Omega)}^{2} \\
& \leq \frac{3}{2}|\alpha| \beta(T)\|\nabla v\|_{L^{2}(\Omega)}^{2}+\frac{|\alpha|}{2 T^{2} \beta(T)}
\end{aligned}
$$

and

$$
\begin{aligned}
\Re\left\langle-A_{\alpha} v, v\right\rangle_{L^{2}(\Omega)} & \leq-\|\nabla v\|_{L^{2}(\Omega)}^{2}+\left|\alpha \Re\left\langle U^{\perp} \operatorname{rot} v, v\right\rangle_{L^{2}(\Omega)}\right| \\
& \leq-(1-|\alpha| \beta(T))\|\nabla v\|_{L^{2}(\Omega)}^{2}+\frac{|\alpha|}{T}\|\nabla v\|_{L^{2}(\Omega)} \\
& \leq-\frac{1-|\alpha| \beta(T)}{2}\|\nabla v\|_{L^{2}(\Omega)}^{2}+\frac{\alpha^{2}}{2 T^{2}(1-|\alpha| \beta(T))} .
\end{aligned}
$$

Combining (248) and (249), we observe that $W\left(-\left.A_{\alpha}\right|_{\mathcal{P}_{n} L_{\sigma}^{2}}\right)$ is included in the set (246), and hence, so is true for the spectrum $\sigma\left(-\left.A_{\alpha}\right|_{\mathcal{P}_{n} L_{\sigma}^{2}}\right)$. The proof is complete. 
The next corollary shows that when $|\alpha|$ is sufficiently small the standard energy method provides a bound of the spectrum except for an exponentially small region (with respect to $|\alpha|)$ near the origin. However, it should be emphasized here that this lack of the information on the spectrum in the exponentially small region is nothing but the essential difficulty in obtaining the stability result as in Theorems 1.1 and 1.3 , for it reflects the absence of the Hardy inequality such as (9) in the two-dimensional case.

Corollary 3.28 Let $|n|=1$. Then for any $\kappa \in\left(0, \frac{1}{2}\right)$ there are constants $\tilde{\delta}_{\kappa}, c_{\kappa} \in(0,1)$ such that if $|\alpha| \leq \tilde{\delta}_{\kappa}$ then the spectrum $\sigma\left(-\left.A_{\alpha}\right|_{\mathcal{P}_{n} L_{\sigma}^{2}}\right)$ is included in the set

$$
\left\{z \in \mathbb{C}|| \Im(z) \mid \leq-\kappa \Re(z)+\kappa \alpha^{2} e^{-\frac{c_{\kappa}}{|\alpha|}}\right\} .
$$

Proof. In Theorem 3.27 let us take $T=e^{\frac{1}{N|\alpha|}}$ with $N \gg 1$ and $|\alpha| \leq N^{-2}$. Then we have from (244),

$$
0<\frac{C_{1}}{N} \leq|\alpha| \beta(T) \leq \frac{C_{2}}{N} \ll \frac{1}{8},
$$

if $N$ is large enough depending on the numerical constant $C_{2}$. Therefore, by Theorem 3.27 the spectrum is included in the set

$$
\begin{gathered}
\left\{z \in \mathbb{C}|\Re(z) \leq 0,| \Im(z) \mid \leq-\frac{6 C_{2}}{N} \Re(z)+\alpha^{2} e^{-\frac{2}{N|\alpha|}}\right\} \\
\cup\left\{z \in \mathbb{C}|\Re(z)>0,| \Im(z) \mid \leq-\frac{3 C_{1}}{N} \Re(z)+\alpha^{2} e^{-\frac{2}{N|\alpha|}}\right\},
\end{gathered}
$$

and thus, for $N \geq \frac{6 C_{2}}{\kappa}$ it is included in the set

$$
\begin{gathered}
\left\{z \in \mathbb{C}|\Re(z) \leq 0,| \Im(z) \mid \leq-\kappa \Re(z)+\alpha^{2} e^{-\frac{2}{N|\alpha|}}\right\} \\
\cup\left\{z \in \mathbb{C}|\Re(z)>0,| \Im(z) \mid \leq-\kappa \Re(z)+\frac{\kappa N}{3 C_{1}} \alpha^{2} e^{-\frac{2}{N|\alpha|}}\right\} .
\end{gathered}
$$

Since $\kappa N \geq 6 C_{2} \geq 3 C_{1}$ we conclude that

$$
\sigma\left(-\left.A_{\alpha}\right|_{\mathcal{P}_{n} L_{\sigma}^{2}}\right) \subset\left\{z \in \mathbb{C}|| \Im(z) \mid \leq-\kappa \Re(z)+\frac{\kappa N}{3 C_{1}} \alpha^{2} e^{-\frac{2}{N|\alpha|}}\right\} .
$$

By the choice of $N$ and $|\alpha|$ we have $e^{-\frac{1}{N|\alpha|}} \leq e^{-N}$, which leads to

$$
\frac{N}{3 C_{1}} e^{-\frac{1}{N|\alpha|}} \leq \frac{N}{3 C_{1}} e^{-N} \leq 1
$$

if $N$ is large enough. Then (252) implies that

$$
\sigma\left(-\left.A_{\alpha}\right|_{\mathcal{P}_{n} L_{\sigma}^{2}}\right) \subset\left\{z \in \mathbb{C}|| \Im(z) \mid \leq-\kappa \Re(z)+\kappa \alpha^{2} e^{-\frac{1}{N|\alpha|}}\right\} .
$$

Here $N=N(\kappa)$ is taken large enough depending only on $\kappa$ and the numerical constants $C_{1}, C_{2}$. Thus the assertion of Corollary 3.28 holds with $c_{\kappa}=N^{-1}$ and $\tilde{\delta}_{\kappa}=N^{-2}$. The proof is complete. 


\subsection{Estimates of $\left\{e^{-t A_{\alpha}}\right\}_{t \geq 0}$ under nonzero condition on $F_{n}(\sqrt{\lambda} ; \alpha)$}

In the next lemma we study the asymptotic behavior of $F_{n}(\sqrt{\lambda} ; \alpha)$ for $|\lambda| \ll 1$. The condition $\alpha \neq 0$ is essentially used, which ensures the property $\mu_{n}=\mu_{n}(\alpha) \notin \mathbb{Z}$.

Lemma 3.29 Let $\alpha \in \mathbb{R} \backslash\{0\}$ and fix $n \in \mathbb{Z} \backslash\{0\}$ and $\epsilon \in\left(0, \frac{\pi}{2}\right)$. Then there is a constant $\delta=\delta(\alpha, n, \epsilon) \in(0,1)$ such that the following statements hold.

(1) For $\lambda \in \Sigma_{\pi-\epsilon} \cap\{z \in \mathbb{C}|| z \mid \leq \delta\}$ the function $F_{n}(\sqrt{\lambda} ; \alpha)$ is expanded as

$$
\left(\mu_{n}+|n|-2\right) F_{n}(\sqrt{\lambda} ; \alpha)=\frac{\pi \lambda^{-\frac{\mu_{n}}{2}}}{2^{1+\mu_{n}} \sin \left(\mu_{n} \pi\right)}\left(\frac{1}{\Gamma\left(-\mu_{n}+1\right)}+o(1)\right), \quad 0<|\lambda| \ll 1 .
$$

(2) For $\lambda \in \Sigma_{\pi-\epsilon} \cap\left\{z \in \mathbb{C}|| z \mid \geq \delta^{-1}\right\}$ the function $F_{n}(\sqrt{\lambda} ; \alpha)$ is expanded as

$$
F_{n}(\sqrt{\lambda} ; \alpha)=\sqrt{\frac{\pi}{2}} \lambda^{-\frac{3}{4}} e^{-\sqrt{\lambda}}\left(1+O\left(|\lambda|^{-\frac{1}{2}}\right)\right), \quad|\lambda| \gg 1 .
$$

Remark 3.30 Let $\epsilon \in\left(0, \frac{\pi}{2}\right)$. Combining the expansion (254) and Theorem 3.24 with Proposition 3.2, we can conclude that for any $\alpha \in \mathbb{R} \backslash\{0\}$ there is $r_{\alpha, \epsilon}>0$ such that $\Sigma_{\pi-\epsilon} \cap B_{r_{\alpha, \epsilon}}(0) \subset \rho\left(-A_{\alpha}\right)$. This fact will be essentially used in the proof of Theorem 1.6 below.

Proof of Lemma 3.29. (1) Firstly we note that $K_{\mu_{n}}(\sqrt{\lambda})$ has an expansion such as

$$
K_{\mu_{n}}(\sqrt{\lambda})=\frac{\pi \lambda^{-\frac{\mu_{n}}{2}}}{2^{1+\mu_{n}} \sin \left(\mu_{n} \pi\right)}\left(\frac{1}{\Gamma\left(-\mu_{n}+1\right)}+O\left(|\lambda|^{\frac{1}{2}}\right)\right), \quad 0<|\lambda| \ll 1
$$

by the identity (53) and the definition of $I_{\mu_{n}}$ in (52). Then, in view of (345), it suffices to show

$$
\sqrt{\lambda} \int_{1}^{\infty} s^{2-|n|} K_{\mu_{n}-1}(s \sqrt{\lambda}) \mathrm{d} s=o\left(|\lambda|^{-\frac{\Re\left(\mu_{n}\right)}{2}}\right), \quad 0<|\lambda| \ll 1 .
$$

We apply Lemmas A.2 and A.3 to obtain for $\lambda \in \Sigma_{\pi-\epsilon}$ with $|\lambda| \ll 1$,

$$
\begin{aligned}
& \left|\sqrt{\lambda} \int_{1}^{\infty} s^{2-|n|} K_{\mu_{n}-1}(s \sqrt{\lambda}) \mathrm{d} s\right| \\
& \leq C|\lambda|^{\frac{1}{2}}\left(\int_{1}^{\frac{1}{\Re(\sqrt{\lambda})}} s^{2-|n|}|\sqrt{\lambda} s|^{-\Re\left(\mu_{n}\right)+1} \mathrm{~d} s+\int_{\frac{1}{\Re(\sqrt{\lambda})}}^{\infty} s^{2-|n|}|\sqrt{\lambda} s|^{-\frac{1}{2}} e^{-s \Re(\sqrt{\lambda})} \mathrm{d} s\right) \\
& \leq C|\lambda|^{-\frac{\Re\left(\mu_{n}\right)}{2}}+1 \int_{1}^{\frac{1}{\Re(\sqrt{\lambda})}} s^{3-|n|-\Re\left(\mu_{n}\right)} \mathrm{d} s+C|\lambda|^{-1+\frac{|n|}{2}}
\end{aligned}
$$

Since $\Re\left(\mu_{n}\right)>|n| \geq 1$ for $\alpha \neq 0$, the estimate (258) implies (257). Hence (254) holds.

(2) By applying (330) we obtain for $|\lambda| \gg 1$,

$$
F_{n}(\sqrt{\lambda} ; \alpha)=\sqrt{\frac{\pi}{2}} \lambda^{-\frac{1}{4}} \int_{1}^{\infty} s^{\frac{1}{2}-|n|} e^{-s \sqrt{\lambda}}\left(1+k_{\mu_{n}}(s \sqrt{\lambda})\right) \mathrm{d} s .
$$

A direct calculation shows that

$$
\int_{1}^{\infty} s^{\frac{1}{2}-|n|} e^{-s \sqrt{\lambda}} \mathrm{d} s=\lambda^{-\frac{1}{2}} e^{-\sqrt{\lambda}}\left(1+O\left(|\lambda|^{-\frac{1}{2}}\right), \quad|\lambda| \gg 1,\right.
$$


while from $\left|k_{\mu_{n}}(s \sqrt{\lambda})\right| \leq C|\lambda|^{-\frac{1}{2}} s^{-1}$,

$$
\begin{aligned}
\left|\int_{1}^{\infty} s^{\frac{1}{2}-|n|} e^{-s \sqrt{\lambda}} k_{\mu_{n}}(s \sqrt{\lambda}) \mathrm{d} s\right| & \leq C|\lambda|^{-\frac{1}{2}} \int_{1}^{\infty} s^{-\frac{1}{2}-|n|} e^{-s \Re(\sqrt{\lambda})} \mathrm{d} s \\
& \leq C|\lambda|^{-1} e^{-\Re(\sqrt{\lambda})} .
\end{aligned}
$$

Hence (255) follows from (259), (260), and (261). The proof is complete.

Proof of Theorem 1.6. Fix $\alpha \in \mathbb{R} \backslash\{0\}$. Let $m=m_{\alpha}$ be the integer satisfying $|\alpha|<m \leq$ $|\alpha|+1$, and set $\theta_{\alpha} \in\left(0, \frac{\pi}{2}\right)$ as $\theta_{\alpha}=\operatorname{Arctan} \frac{|\alpha|}{m-|\alpha|}$. We recall that $A_{\alpha}$ is diagonalized as

$$
A_{\alpha}=\left.\left.A\right|_{L_{\sigma}^{2}, \text { rad }} \oplus A_{\alpha}\right|_{\mathcal{Q}_{m} L_{\sigma}^{2}} \oplus\left(\left.\oplus_{1 \leq|n| \leq m} A_{\alpha}\right|_{\mathcal{P}_{n} L_{\sigma}^{2}}\right) .
$$

For the part $\left.A\right|_{L_{\sigma}^{2} \text {,rad }}$ we have nothing to prove, for it is just the Stokes operator in the invariant subspace $\mathcal{P}_{0} L_{\sigma}^{2}(\Omega)$, which is a nonnegative self-adjoint operator satisfying all estimates of (16), (7), and (8). As for the part $\left.A_{\alpha}\right|_{\mathcal{Q}_{m} L_{\sigma}^{2}}$, since $|\alpha|<m$ holds by the choice of $m$, the statement (1) of Theorem 3.24 implies that the sector $\Sigma_{\pi-\theta_{\alpha}}$ is included in the resolvent set $\rho\left(-\left.A_{\alpha}\right|_{\mathcal{Q}_{m} L_{\sigma}^{2}}\right)$. Moreover, the resolvent estimate (230) for $-\left.A_{\alpha}\right|_{\mathcal{Q}_{m} L_{\sigma}^{2}}$ in $\Sigma_{\pi-\theta_{\alpha}}$ yields that

$$
\left\|\left(\lambda+\left.A_{\alpha}\right|_{\mathcal{Q}_{m} L_{\sigma}^{2}}\right)^{-1} v\right\|_{L^{2}(\Omega)} \leq \frac{C}{|\lambda|}\|v\|_{L^{2}(\Omega)}, \quad \lambda \in \Sigma_{\pi-\epsilon}, \quad v \in \mathcal{Q}_{m} L_{\sigma}^{2}(\Omega)
$$

for any $\epsilon \in\left(\theta_{\alpha}, \frac{\pi}{2}\right)$, where $C$ depends only on $\alpha$ and $\epsilon$. We have already proved the estimates (7) and (8) for $\left\{\left.e^{-t A_{\alpha}}\right|_{\mathcal{Q}_{m} L_{\sigma}^{2}}\right\}_{t \geq 0}$ in Theorem 3.24.

It remains to consider the part $\left.A_{\alpha}\right|_{\mathcal{P}_{n} L_{\sigma}^{2}}$ with $1 \leq|n| \leq m$. If the assumption (15) holds for some $\epsilon_{0} \in\left(\theta_{\alpha}, \frac{\pi}{2}\right)$ then the sector $\Sigma_{\pi-\epsilon_{0}}$ is included in the resolvent set $\rho\left(-\left.A_{\alpha}\right|_{\mathcal{P}_{n} L_{\sigma}^{2}}\right)$ with $1 \leq|n| \leq m$ by Propositions 2.12 and 3.2. Thus it suffices to show the estimates such as (16), (7), and (8) for $\left.A_{\alpha}\right|_{\mathcal{P}_{n} L_{\sigma}^{2}}$ with $1 \leq|n| \leq m$. Let $1 \leq q<p \leq \infty$ or $1<q \leq p \leq \infty$, and let $f_{n} \in \mathcal{P}_{n} C_{0, \sigma}^{\infty}(\Omega)$. By Theorem 3.19 and Lemma 3.29 there is $\delta \in(0,1)$ such that

$$
\left\|\left(\lambda+\left.A_{\alpha}\right|_{\mathcal{P}_{n} L_{\sigma}^{2}}\right)^{-1} f_{n}\right\|_{L^{p}(\Omega)} \leq \frac{C}{|\lambda|^{1+\frac{1}{p}-\frac{1}{q}}}\left\|f_{n}\right\|_{L^{q}(\Omega)},
$$

holds for all $\lambda \in \Sigma_{\pi-\epsilon_{0}}$ satisfying either $|\lambda| \leq \delta$ or $|\lambda| \geq \delta^{-1}$. On the other hand, since $\left|F_{n}(\sqrt{\lambda} ; \alpha)\right|$ is continuous with respect to $\lambda$ in $\mathbb{C} \backslash \overline{\mathbb{R}_{-}}$and since there are no zero points of $F_{n}(\sqrt{\lambda} ; \alpha)$ in $\bar{\Sigma}_{\pi-\epsilon_{0}}$ by the assumption, there is $\kappa>0$ such that

$$
\left|F_{n}(\sqrt{\lambda} ; \alpha)\right| \geq \kappa \quad \text { for all } \lambda \in \bar{\Sigma}_{\pi-\epsilon_{0}} \text { satisfying } \delta \leq|\lambda| \leq \delta^{-1} .
$$

Hence we conclude from Theorem 3.19 that (263) holds for all $\lambda \in \Sigma_{\pi-\epsilon_{0}}$. In particular, we obtain (263) with $p=q=2$ for all $f_{n} \in \mathcal{P}_{n} L_{\sigma}^{2}(\Omega)$, which proves (16), and thus the $C_{0}$-analyic semigroup $\left\{\left.e^{-t A_{\alpha}}\right|_{\mathcal{P}_{n} L_{\sigma}^{2}}\right\}_{t \geq 0}$ defines a bounded semigroup in $\mathcal{P}_{n} L_{\sigma}^{2}(\Omega)$, i.e.,

$$
\left\|e^{-t A_{\alpha}} f_{n}\right\|_{L^{2}(\Omega)} \leq C\left\|f_{n}\right\|_{L^{2}(\Omega)}, \quad t>0, \quad f_{n} \in \mathcal{P}_{n} L_{\sigma}^{2}(\Omega) .
$$

The similar uniform bound is valid also in $L_{\sigma}^{q}(\Omega)$ with $1<q<\infty$. To obtain the $L^{p}-L^{q}$ estimates of $\left\{\left.e^{-t A_{\alpha}}\right|_{\mathcal{P}_{n} L_{\sigma}^{2}}\right\}_{t \geq 0}$ for $1 \leq q<p \leq \infty$ we use a representation of $\left.e^{-t A_{\alpha}}\right|_{\mathcal{P}_{n} L_{\sigma}^{2}}$ in the Dunford integral

$$
\left.e^{-t A_{\alpha}}\right|_{\mathcal{P}_{n} L_{\sigma}^{2}}=\frac{1}{2 \pi i} \int_{\gamma} e^{t \lambda}\left(\lambda+\left.A_{\alpha}\right|_{\mathcal{P}_{n} L_{\sigma}^{2}}\right)^{-1} \mathrm{~d} \lambda, \quad t>0
$$


Here $\gamma=\gamma_{b}$ is the curve $\{\lambda \in \mathbb{C}|| \arg \lambda|=\eta,| \lambda \mid \geq b\} \cup\{\lambda \in \mathbb{C}|| \arg \lambda|\leq \eta,| \lambda \mid=b\}$ for some $\eta \in\left(\frac{\pi}{2}, \pi\right)$ and $0<b \ll 1$, oriented counterclockwise, and $\eta$ is taken so that $\gamma$ belongs to the sector $\Sigma_{\pi-\epsilon_{0}}$. We denote by $\gamma_{0}$ the limit curve obtained by taking $b=0$. Then (263) yields for any $f_{n} \in \mathcal{P}_{n} C_{0, \sigma}^{\infty}(\Omega)$ and $1 \leq q<p \leq \infty$,

$$
\begin{aligned}
\left\|e^{-t A_{\alpha}} f_{n}\right\|_{L^{p}(\Omega)} & \leq C \int_{\gamma_{0}}|\lambda|^{-1-\frac{1}{p}+\frac{1}{q}} e^{t \Re(\lambda)}|\mathrm{d} \lambda|\left\|f_{n}\right\|_{L^{q}(\Omega)} \\
& \leq C \int_{0}^{\infty} r^{-1-\frac{1}{p}+\frac{1}{q}} e^{-t r \cos \epsilon_{0}} \mathrm{~d} r\left\|f_{n}\right\|_{L^{q}(\Omega)} \\
& \leq C t^{-\frac{1}{q}+\frac{1}{p}}\left\|f_{n}\right\|_{L^{q}(\Omega)}, \quad t>0 .
\end{aligned}
$$

By the density argument the estimate (267) is extended to all $f_{n} \in \mathcal{P}_{n} L_{\sigma}^{q}(\Omega)$, and the estimate (7) has been proved. Arguing similarly, we have from Theorem 3.23 and Lemma 3.29 that

$$
\left\|\nabla\left(\lambda+\left.A_{\alpha}\right|_{\mathcal{P}_{n} L_{\sigma}^{2}}\right)^{-1} f_{n}\right\|_{L^{2}(\Omega)} \leq \frac{C}{|\lambda|^{\frac{1}{2}}}\left\|f_{n}\right\|_{L^{2}(\Omega)}, \quad \lambda \in \Sigma_{\pi-\epsilon_{0}}, \quad f_{n} \in \mathcal{P}_{n} L_{\sigma}^{2}(\Omega),
$$

which leads to

$$
\left\|\nabla e^{-t A_{\alpha}} f_{n}\right\|_{L^{2}(\Omega)} \leq C t^{-\frac{1}{2}}\left\|f_{n}\right\|_{L^{2}(\Omega)}, \quad t>0
$$

by using (266) as in the derivation of (267). Then (8) with $q \in[1,2)$ follows from (267) and (269) due to the semigroup property. The proof of Theorem 1.6 is complete.

\subsection{Analysis of zero points of $F_{n}(z ; \alpha)$ with $|n|=1$ for $|\alpha| \ll 1$}

The aim of this section is to show the nonexistence of zero points of $F_{n}(\sqrt{\lambda} ; \alpha)$ for the case $|n|=1$ and $|\alpha| \ll 1$ in a certain sector, which leads to Theorem 1.3 by virtue of Theorem 1.6. By Corollary 3.28 we have already shown that there are no zero points of $F_{n}(\sqrt{\lambda} ; \alpha)$ in the domain $\left\{\lambda \in \mathbb{C}|| \Im(\lambda) \mid>-\kappa \Re(\lambda)+\kappa \alpha^{2} e^{-\frac{c_{\kappa}}{|\alpha|}}\right\}$ for $\kappa \in\left(0, \frac{1}{2}\right)$ and $|\alpha| \leq \tilde{\delta}_{\kappa} \ll 1$. Hence it suffices to focus on a small domain close to the origin at most, e.g., in some algebraic order such as $|\lambda| \leq|\alpha|^{k}$ for some $k>0$. To this end we need a delicate asymptotic analysis based on the known asymptotic expansion of the modified Bessel function. Let us recall that $\mu_{n}(\alpha)=\left(n^{2}+i \alpha n\right)^{\frac{1}{2}}$, which satisfies $\Re\left(\mu_{n}(\alpha)\right)>|n|$ for $\alpha \neq 0$. We start from the following lemma.

Lemma 3.31 Let $|n|=1$ and set $\zeta_{n}=\zeta_{n}(\alpha)=\mu_{n}(\alpha)-1$. Fix $\epsilon \in\left(0, \frac{\pi}{2}\right)$. Then for any $z \in \Sigma_{\pi-\epsilon}$ it follows that

$$
\begin{aligned}
K_{\mu_{n}}(z)= & \frac{\Gamma\left(1+\zeta_{n}\right)}{z}\left(\frac{z}{2}\right)^{-\zeta_{n}} \\
- & \frac{\pi}{2} \sum_{m=0}^{\infty} \frac{1}{(m+1) ! \Gamma\left(m+1-\zeta_{n}\right) \Gamma\left(m+2+\zeta_{n}\right)} \\
& \quad \times \frac{\Gamma\left(m+2+\zeta_{n}\right)-(m+1) \Gamma\left(m+1-\zeta_{n}\right)}{\sin \left(\zeta_{n} \pi\right)}\left(\frac{z}{2}\right)^{2 m+1-\zeta_{n}} \\
- & \frac{\pi}{2} \sum_{m=0}^{\infty} \frac{1}{m ! \Gamma\left(m+2+\zeta_{n}\right)} \frac{1}{\sin \left(\zeta_{n} \pi\right)}\left\{\left(\frac{z}{2}\right)^{2 m+1-\zeta_{n}}-\left(\frac{z}{2}\right)^{2 m+1+\zeta_{n}}\right\} .
\end{aligned}
$$


In particular, if $|\alpha| \ll \frac{1}{2}$ then for any $z \in \Sigma_{\pi / 2}$ with $|z| \leq \frac{1}{2}$ the following expansion holds:

$$
K_{\mu_{n}}(z)=\frac{\Gamma\left(1+\zeta_{n}\right)}{z}\left(\frac{z}{2}\right)^{-\zeta_{n}}+R_{n, \alpha}^{(1)}(z)
$$

with

$$
\left|R_{n, \alpha}^{(1)}(z)\right| \leq-C|z|^{1-\Re\left(\zeta_{n}\right)} \log |z| .
$$

Here $C$ is a positive constant independent of $\alpha$ and $z$.

Proof. By the definition of $I_{\mu}(z)$ in (52) and the relation (53) we see

$$
\begin{aligned}
& K_{\mu_{n}}(z) \\
& =\frac{\pi}{2} \frac{1}{\sin \left(\mu_{n} \pi\right)}\left\{\sum_{m=0}^{\infty} \frac{1}{m ! \Gamma\left(-\mu_{n}+m+1\right)}\left(\frac{z}{2}\right)^{2 m-\mu_{n}}-\sum_{m=0}^{\infty} \frac{1}{m ! \Gamma\left(\mu_{n}+m+1\right)}\left(\frac{z}{2}\right)^{2 m+\mu_{n}}\right\} \\
& =-\frac{\pi}{2} \frac{1}{\sin \left(\zeta_{n} \pi\right) \Gamma\left(-\zeta_{n}\right)}\left(\frac{z}{2}\right)^{-1-\zeta_{n}} \\
& -\frac{\pi}{2} \frac{1}{\sin \left(\zeta_{n} \pi\right)} \sum_{m=0}^{\infty}\left\{\frac{1}{(m+1) ! \Gamma\left(m+1-\zeta_{n}\right)}\left(\frac{z}{2}\right)^{2 m+1-\zeta_{n}}-\frac{1}{m ! \Gamma\left(m+2+\zeta_{n}\right)}\left(\frac{z}{2}\right)^{2 m+1+\zeta_{n}}\right\}
\end{aligned}
$$

Then (270) follows by using the identity

$$
\Gamma(-s) \Gamma(1+s)=-\frac{\pi}{\sin (\pi s)}
$$

for the first term in the right-hand side of (273). The estimates (272) is derived from the fact $\lim _{\alpha \rightarrow 0} \zeta_{n}(\alpha)=0$ by Lemma B.1 and the estimates for $0<\left|\zeta_{n}\right| \leq \frac{1}{4}$ such as

$$
\begin{aligned}
& \left|\frac{\Gamma\left(m+2+\zeta_{n}\right)-(m+1) \Gamma\left(m+1-\zeta_{n}\right)}{\sin \left(\zeta_{n} \pi\right)}\right| \\
& =\left|\frac{\left(m+1+\zeta_{n}\right) \Gamma\left(m+1+\zeta_{n}\right)-(m+1) \Gamma\left(m+1-\zeta_{n}\right)}{\sin \left(\zeta_{n} \pi\right)}\right| \leq C(m+1) !,
\end{aligned}
$$

and

$$
\begin{aligned}
\left|\left(\frac{z}{2}\right)^{2 m+1-\zeta_{n}}\right| & \leq C|z|^{2 m+1-\Re\left(\zeta_{n}\right)}, \\
\left|\frac{1}{\sin \left(\zeta_{n} \pi\right)}\left\{\left(\frac{z}{2}\right)^{2 m+1-\zeta_{n}}-\left(\frac{z}{2}\right)^{2 m+1+\zeta_{n}}\right\}\right| & \leq C|z|^{2 m+1-\Re\left(\zeta_{n}\right)}\left|\log \frac{z}{2}\right| .
\end{aligned}
$$

Here, the first inequality can be shown by using the expression of the Gamma function $\Gamma(\xi)=\int_{0}^{\infty} e^{-t} t^{\xi-1} \mathrm{~d} t$ for $\Re(\xi)>0$. In the last inequality the function $\log z=\log |z|+$ $i \arg z,-\pi<\arg z<\pi$, is the principal branch of the logarithm of $z$ in $\Sigma_{\pi-\epsilon}$. The proof is complete.

Lemma 3.32 Let $|n|=1$ and set $\zeta_{n}(\alpha)=\mu_{n}(\alpha)-1$. If $|\alpha| \ll \frac{1}{2}$ then for any $z \in \Sigma_{\pi / 2}$ the following identity holds:

$$
\int_{0}^{\infty} \frac{1}{t^{\zeta_{n}}\left(t^{2}+1\right)}\left(1+\frac{2 t}{z\left(t^{2}+1\right)}\right) e^{-\frac{z}{2}\left(t+\frac{1}{t}\right)} \mathrm{d} t=\frac{1}{z} \frac{\frac{\pi}{2} \zeta_{n}}{\sin \left(\frac{\pi}{2} \zeta_{n}\right)}+R_{n, \alpha}^{(2)}(z),
$$


where $R_{n, \alpha}^{(2)}(z)$ satisfies

$$
\left|R_{n, \alpha}^{(2)}(z)\right| \leq C .
$$

Here $C$ is a positive constant independent of $\alpha$ and $z$ such that $|\alpha| \ll \frac{1}{2}$ and $z \in \Sigma_{\pi / 2}$.

Proof. We rewrite the left-hand side of (274) as

$$
\text { (L.H.S) of (274) }=\frac{2}{z} \int_{0}^{\infty} \frac{t^{1-\zeta_{n}}}{\left(t^{2}+1\right)^{2}} \mathrm{~d} t+R_{n, \alpha}^{(2)}(z),
$$

where

$$
R_{n, \alpha}^{(2)}(z)=\frac{2}{z} \int_{0}^{\infty} \frac{t^{1-\zeta_{n}}}{\left(t^{2}+1\right)^{2}}\left(e^{-\frac{z}{2}\left(t+\frac{1}{t}\right)}-1\right) \mathrm{d} t+\int_{0}^{\infty} \frac{1}{t^{\zeta_{n}}\left(t^{2}+1\right)} e^{-\frac{z}{2}\left(t+\frac{1}{t}\right)} \mathrm{d} t .
$$

Then we observe that

$$
\begin{aligned}
2 \int_{0}^{\infty} \frac{t^{1-\zeta_{n}}}{\left(t^{2}+1\right)^{2}} \mathrm{~d} t & =\int_{0}^{\infty} \frac{\tau^{-\frac{\zeta n}{2}}}{(1+\tau)^{2}} \mathrm{~d} \tau=B\left(1-\frac{\zeta_{n}}{2}, 1+\frac{\zeta_{n}}{2}\right) \\
& =\frac{\Gamma\left(1-\frac{\zeta_{n}}{2}\right) \Gamma\left(1+\frac{\zeta_{n}}{2}\right)}{\Gamma(2)} \\
& =\Gamma\left(1-\frac{\zeta_{n}}{2}\right) \Gamma\left(\frac{\zeta_{n}}{2}\right) \frac{\zeta_{n}}{2}=\frac{\frac{\pi}{2} \zeta_{n}}{\sin \left(\frac{\pi}{2} \zeta_{n}\right)}
\end{aligned}
$$

Here $B(x, y)=\int_{0}^{1} t^{x-1}(1-t)^{y-1} \mathrm{~d} t$ is the Beta function and $\Gamma(x)$ is the Gamma function, while we have used the well-known formulas

$$
B(x, y)=\frac{\Gamma(x) \Gamma(y)}{\Gamma(x+y)}, \quad \Gamma(1+x)=x \Gamma(x), \quad \Gamma(x) \Gamma(1-x)=\frac{\pi}{\sin (\pi x)} .
$$

It remains to estimate $R_{n, \alpha}^{(2)}(z)$. When $\Re(z)>0$ we have

$$
\left|e^{-\frac{z}{2}\left(t+\frac{1}{t}\right)}\right| \leq 1, \quad\left|e^{-\frac{z}{2}\left(t+\frac{1}{t}\right)}-1\right| \leq|z|\left(t+\frac{1}{t}\right),
$$

which yields for $|\alpha| \ll \frac{1}{2}$,

$$
\left|R_{n, \alpha}^{(2)}(z)\right| \leq 3 \int_{0}^{\infty} t^{-\Re\left(\zeta_{n}\right)}\left(t^{2}+1\right)^{-1} \mathrm{~d} t \leq C,
$$

since $\zeta_{n}(\alpha) \rightarrow 0$ as $\alpha \rightarrow 0$. In particular, the constant $C$ is independent of $\alpha$ and $z$ such that $|\alpha| \ll \frac{1}{2}$ and $z \in \Sigma_{\pi / 2}$. The proof is complete.

Lemma 3.33 There is $\delta>0$ such that for any $\zeta \in \mathbb{C}$ with $|\zeta| \leq \delta$ we have

$$
\log \left(\frac{\sin \left(\frac{\zeta}{2} \pi\right)}{\frac{\zeta}{2} \pi} \Gamma(1+\zeta)\right)=-\zeta \gamma(\zeta),
$$

where $\gamma(\zeta)$ satisfies

$$
\gamma(\zeta)=\gamma+O(|\zeta|), \quad|\zeta| \rightarrow 0
$$

with the Euler constant $\gamma=0.57721 \cdots$. 
Proof. When $|\zeta|$ is small enough both $\frac{\sin \left(\frac{\zeta}{2} \pi\right)}{\frac{\zeta}{2} \pi}$ and $\Gamma(1+\zeta)$ are close to 1 , which gives

$$
\log \left(\frac{\sin \left(\frac{\zeta}{2} \pi\right)}{\frac{\zeta}{2} \pi} \Gamma(1+\zeta)\right)=\log \left(\frac{\sin \left(\frac{\zeta}{2} \pi\right)}{\frac{\zeta}{2} \pi}\right)+\log (\Gamma(1+\zeta)) .
$$

Now let us recall that product formulas

$$
\begin{aligned}
\sin z & =z \prod_{m=1}^{\infty}\left(1-\frac{z^{2}}{m^{2} \pi^{2}}\right), \\
\frac{1}{\Gamma(1+\zeta)} & =(1+\zeta) e^{\gamma(1+\zeta)} \prod_{m=1}^{\infty}\left(1+\frac{1+\zeta}{m}\right) e^{-\frac{1+\zeta}{m}},
\end{aligned}
$$

which shows for $|\zeta| \ll 1$,

$$
\begin{aligned}
& \log \left(\frac{\sin \left(\frac{\zeta}{2} \pi\right)}{\frac{\zeta}{2} \pi}\right)=\sum_{m=1}^{\infty} \log \left(1-\frac{\zeta^{2}}{4 m^{2}}\right) \\
& \log (\Gamma(1+\zeta))=-\log (1+\zeta)-\gamma(1+\zeta)-\sum_{m=1}^{\infty}\left\{\log \left(1+\frac{1+\zeta}{m}\right)-\frac{1+\zeta}{m}\right\}
\end{aligned}
$$

Next we use the expansion

$$
\log (1+z)=\sum_{k=1}^{\infty} \frac{(-1)^{k-1}}{k} z^{k}, \quad|z|<1 .
$$

Firstly (279) and (281) yield

$$
\left|\log \left(\frac{\sin \left(\frac{\zeta}{2} \pi\right)}{\frac{\zeta}{2} \pi}\right)\right| \leq \sum_{m=1}^{\infty}\left(\frac{|\zeta|^{2}}{4 m^{2}}+O\left(\frac{|\zeta|^{4}}{m^{4}}\right)\right) \leq C|\zeta|^{2} .
$$

To estimate (280) we also recall the formula for the Euler constant

$$
\gamma=\sum_{m=1}^{\infty}\left\{\frac{1}{m}-\log \left(1+\frac{1}{m}\right)\right\}
$$

which implies

$$
\log (\Gamma(1+\zeta))=-\log (1+\zeta)-\gamma \zeta-\sum_{m=1}^{\infty}\left\{\log \left(1+\frac{\zeta}{m+1}\right)-\frac{\zeta}{m}\right\} .
$$

Then (281) and (283) lead to

$$
\begin{aligned}
\log (\Gamma(1+\zeta)) & =-\log (1+\zeta)-\gamma \zeta-\sum_{m=1}^{\infty}\left\{\frac{\zeta}{m+1}-\frac{\zeta}{m}+O\left(\frac{|\zeta|^{2}}{(m+1)^{2}}\right)\right\} \\
& =-\log (1+\zeta)-\gamma \zeta+\zeta+O\left(|\zeta|^{2}\right) \\
& =-\gamma \zeta+O\left(|\zeta|^{2}\right) .
\end{aligned}
$$

Collecting (278), (282), and (284), we obtain (276)-(277). The proof is complete.

We are now in position to prove the nonexistence of zero points of $F_{n}(z ; \alpha)$ with $|n|=1$ and $|\alpha| \ll 1$ in an algebraically small region $|z| \leq|\alpha|^{2}$. 
Proposition 3.34 Let $|n|=1$. Then for any $\epsilon \in\left(0, \frac{\pi}{2}\right)$ there is $\delta_{\epsilon}>0$ such that if $|\alpha| \leq \delta_{\epsilon}$ then $F_{n}(z ; \alpha) \neq 0$ for $z \in \Sigma_{\frac{\pi}{2}-\epsilon} \cap B_{\alpha^{2}}(0)$. Here $B_{r}(0)=\{z \in \mathbb{C}|| z \mid<r\}$.

Proof. We will prove the assertion by a contradiction argument. Let $|n|=1$ and assume that there exists $z_{0} \in \Sigma_{\frac{\pi}{2}-\epsilon} \cap B_{\alpha^{2}}(0)$ such that $F_{n}\left(z_{0} ; \alpha\right)=0$. Set $\zeta_{n}=\zeta_{n}(\alpha)=\mu_{n}(\alpha)-1$. Note that $\lim _{\alpha \rightarrow 0} \zeta_{n}=0$ holds by (347). From the hypothesis $F_{n}\left(z_{0} ; \alpha\right)=0$, Corollary A.8 implies

$$
K_{\mu_{n}}\left(z_{0}\right)=\int_{0}^{\infty} \frac{1}{t^{\mu_{n}-1}\left(t^{2}+1\right)}\left(1+\frac{2 t}{z_{0}\left(t^{2}+1\right)}\right) e^{-\frac{z_{0}}{2}\left(t+\frac{1}{t}\right)} \mathrm{d} t
$$

Then Lemma 3.31 and Lemma 3.32 yield

$$
\frac{\Gamma\left(1+\zeta_{n}\right)}{z_{0}}\left(\frac{z_{0}}{2}\right)^{-\zeta_{n}}=\frac{1}{z_{0}} \frac{\frac{\pi}{2} \zeta_{n}}{\sin \left(\frac{\pi}{2} \zeta_{n}\right)}-R_{n, \alpha}^{(1)}\left(z_{0}\right)+R_{n, \alpha}^{(2)}\left(z_{0}\right) .
$$

That is, for small $|\alpha|$,

$$
\begin{aligned}
& \left\{\frac{\sin \left(\frac{\pi}{2} \zeta_{n}\right)}{\frac{\pi}{2} \zeta_{n}} \Gamma\left(1+\zeta_{n}\right)\left(\frac{z_{0}}{2}\right)^{-\zeta_{n}}-1\right\} \frac{1}{z_{0}}=R_{n, \alpha}^{(3)}\left(z_{0}\right), \\
& \left|R_{n, \alpha}^{(3)}\left(z_{0}\right)\right|=\left|\frac{\sin \left(\frac{\pi}{2} \zeta_{n}\right)}{\frac{\pi}{2} \zeta_{n}}\left(-R_{n, \alpha}^{(1)}\left(z_{0}\right)+R_{n, \alpha}^{(2)}\left(z_{0}\right)\right)\right| \leq C
\end{aligned}
$$

with $C$ independent of $|\alpha| \ll 1$ and $\left|z_{0}\right| \leq \alpha^{2} \leq \frac{1}{2}$. Here we have used

$$
\frac{1}{2} \leq\left|\frac{\sin \left(\frac{\pi}{2} \zeta_{n}\right)}{\frac{\pi}{2} \zeta_{n}}\right| \leq 2
$$

for sufficiently small $\left|\zeta_{n}\right|$. Let $\gamma\left(\zeta_{n}\right)$ be the function in Lemma 3.33, and set

$$
\tilde{z}_{0}=\tilde{z}_{0}\left(\zeta_{n}\right)=\frac{e^{\gamma\left(\zeta_{n}\right)}}{2} z_{0}
$$

Since $\gamma\left(\zeta_{n}\right)=\gamma+O\left(\left|\zeta_{n}\right|\right)$ for $\left|\zeta_{n}\right| \ll 1$ and $z_{0} \in \Sigma_{\frac{\pi}{2}-\epsilon}$, we have

$$
c_{1} \leq\left|\frac{\tilde{z}_{0}}{z_{0}}\right| \leq c_{2}, \quad\left|\arg \tilde{z}_{0}\right|<\frac{\pi}{2}-\frac{\epsilon}{2}
$$

for some positive constants $c_{1}$ and $c_{2}$ independent of $|\alpha| \ll 1$ and $\left|z_{0}\right| \leq \alpha^{2}$. Using the identity

$$
\frac{\sin \left(\frac{\pi}{2} \zeta_{n}\right)}{\frac{\pi}{2} \zeta_{n}} \Gamma\left(1+\zeta_{n}\right)\left(\frac{z_{0}}{2}\right)^{-\zeta_{n}}=\left(\frac{e^{\gamma\left(\zeta_{n}\right)}}{2} z_{0}\right)^{-\zeta_{n}}=\tilde{z}_{0}^{-\zeta_{n}},
$$

we have from (285) and (287),

$$
\left(\tilde{z}_{0}^{-\zeta_{n}}-1\right) \frac{1}{\tilde{z}_{0}}=R_{n, \alpha}^{(4)}\left(z_{0}\right), \quad\left|R_{n, \alpha}^{(4)}\left(z_{0}\right)\right| \leq C
$$

with $C$ independent of $|\alpha| \ll 1$ and $\left|z_{0}\right| \leq \alpha^{2}$. We set

$$
g\left(\zeta_{n}, \tilde{z}_{0}\right)=-\Re\left(\zeta_{n}\right) \log \left|\tilde{z}_{0}\right|+\Im\left(\zeta_{n}\right) \theta\left(\tilde{z}_{0}\right), \quad \theta\left(\tilde{z}_{0}\right)=\arg \tilde{z}_{0} .
$$


The identity (289) is also written as $\log \left|\tilde{z}_{0}\right|=-\frac{1}{\Re\left(\zeta_{n}\right)} g\left(\zeta_{0}, \tilde{z}_{0}\right)+\frac{\Im\left(\zeta_{n}\right)}{\Re\left(\zeta_{n}\right)} \theta\left(\tilde{z}_{0}\right)$, which yields

$$
\tilde{z}_{0}^{-\zeta_{n}}=e^{-\zeta_{n} \log \tilde{z}_{0}}=e^{g\left(\zeta_{n}, \tilde{z}_{0}\right)} e^{i \omega\left(\zeta_{n}, \tilde{z}_{0}\right)},
$$

where

$$
\omega\left(\zeta_{n}, \tilde{z}_{0}\right)=-\left(\Re\left(\zeta_{n}\right)+\frac{\Im\left(\zeta_{n}\right)^{2}}{\Re\left(\zeta_{n}\right)}\right) \theta\left(\tilde{z}_{0}\right)+\frac{\Im\left(\zeta_{n}\right)}{\Re\left(\zeta_{n}\right)} g\left(\zeta_{n}, \tilde{z}_{0}\right) .
$$

Note that Lemma B.1 implies

$$
\frac{\Im\left(\zeta_{n}\right)^{2}}{\Re\left(\zeta_{n}\right)}=2+O\left(|\alpha|^{2}\right), \quad|\alpha| \ll 1 .
$$

(i) Case $\left|g\left(\zeta_{n}, \tilde{z}_{0}\right)\right| \leq \kappa\left|\Im\left(\zeta_{n}\right)\right|\left|\theta\left(\tilde{z}_{0}\right)\right|$ for $0<\kappa \ll \frac{1}{2}$ : In this case we have from (347), (287), and (292),

$$
\left|\omega\left(\zeta_{n}, \tilde{z}_{0}\right)\right| \leq\left(\Re\left(\zeta_{n}\right)+(1+\kappa) \frac{\Im\left(\zeta_{n}\right)^{2}}{\Re\left(\zeta_{n}\right)}\right)\left|\theta\left(\tilde{z}_{0}\right)\right|<\pi,
$$

if $|\alpha|$ and $\kappa$ are sufficiently small. Here the smallness of $\kappa$ can be chosen depending only on $\epsilon$ as long as $|\alpha| \leq \delta_{\epsilon} \ll 1$. We fix this $\kappa$ in the argument below. The estimate (293) implies that $e^{i \omega\left(\zeta_{n}, \tilde{z}_{0}\right)}$ can be close to 1 only when $\omega\left(\zeta_{n}, \tilde{z}_{0}\right)$ is close to 0 . On the other hand, the identity (289) and the present condition (i) lead to

$$
\left|-\Re\left(\zeta_{n}\right) \log \right| \tilde{z}_{0}|| \leq(1+\kappa)\left|\Im\left(\zeta_{n}\right)\right|\left|\theta\left(\tilde{z}_{0}\right)\right|,
$$

and hence, Lemma B.1 and (287) give

$$
\left|\theta\left(\tilde{z}_{0}\right)\right| \geq-\frac{1}{1+\kappa}\left|\frac{\Re\left(\zeta_{n}\right)}{\Im\left(\zeta_{n}\right)}\right| \log \left|\tilde{z}_{0}\right| \geq-c|\alpha| \log |\alpha|,
$$

as long as $\left|\tilde{z}_{0}\right| \leq c_{2}\left|z_{0}\right| \leq c_{2} \alpha^{2}$. Thus for $|\alpha| \ll 1$ we have from (291),

$$
\begin{aligned}
\left|\omega\left(\zeta_{n}, \tilde{z}_{0}\right)\right| & \geq\left(\Re\left(\zeta_{n}\right)+\frac{\Im\left(\zeta_{n}\right)^{2}}{\Re\left(\zeta_{n}\right)}\right)\left|\theta\left(\tilde{z}_{0}\right)\right|-\left|\frac{\Im\left(\zeta_{n}\right)}{\Re\left(\zeta_{n}\right)}\right|\left|g\left(\zeta_{n}, \tilde{z}_{0}\right)\right| \\
& \geq\left(\Re\left(\zeta_{n}\right)+(1-\kappa) \frac{\Im\left(\zeta_{n}\right)^{2}}{\Re\left(\zeta_{n}\right)}\right)\left|\theta\left(\tilde{z}_{0}\right)\right| \\
& \geq\left|\theta\left(\tilde{z}_{0}\right)\right| \geq-c|\alpha| \log |\alpha| .
\end{aligned}
$$

Here we have also used Lemma B.1, (292), and (294). Since $\frac{3}{4} \leq e^{g\left(\zeta_{n}, \tilde{z}_{0}\right)} \leq \frac{5}{4}$ in the present case (i), the identity (290) combined with the estimates (293) and (295) implies

$$
\begin{aligned}
\left|\tilde{z}_{0}^{-\zeta_{n}}-1\right| & =\left|e^{g\left(\zeta_{n}, \tilde{z}_{0}\right)} \cos \left(\omega\left(\zeta_{n}, \tilde{z}_{0}\right)\right)-1+i e^{g\left(\zeta_{n}, \tilde{z}_{0}\right)} \sin \left(\omega\left(\zeta_{n}, \tilde{z}_{0}\right)\right)\right| \\
& \geq-c^{\prime}|\alpha| \log |\alpha|
\end{aligned}
$$

for some $c^{\prime}>0$ independent of $|\alpha| \ll 1$ and $\left|\tilde{z}_{0}\right| \leq c_{2} \alpha^{2}$. Therefore, we have

$$
\left|\left(\tilde{z}_{0}^{-\zeta_{n}}-1\right) \frac{1}{\tilde{z}_{0}}\right| \geq \frac{-c^{\prime}|\alpha| \log |\alpha|}{c_{2}|\alpha|^{2}}=-\frac{c^{\prime}}{c_{2}|\alpha|} \log |\alpha|,
$$

which goes to $\infty$ as $|\alpha| \rightarrow 0$ and thus contradicts with (288) if $|\alpha|$ is small enough. 
(ii) Case $\left|g\left(\zeta_{n}, \tilde{z}_{0}\right)\right| \geq \kappa\left|\Im\left(\zeta_{n}\right)\right|\left|\theta\left(\tilde{z}_{0}\right)\right|$, where $\kappa \in\left(0, \frac{1}{2}\right)$ is fixed as in (i): We further divide into two cases: (1) $\left|\theta\left(\tilde{z}_{0}\right)\right| \leq-\frac{1}{2} \frac{\Re\left(\zeta_{n}\right)}{\left|\Im\left(\zeta_{n}\right)\right|} \log \left|\tilde{z}_{0}\right|$ and (2) $\left|\theta\left(\tilde{z}_{0}\right)\right|>-\frac{1}{2} \frac{\Re\left(\zeta_{n}\right)}{\left|\Im\left(\zeta_{n}\right)\right|} \log \left|\tilde{z}_{0}\right|$. In the case (1) we have from (289),

$$
\left|g\left(\zeta_{n} ; \tilde{z}_{0}\right)\right|>-\Re\left(\zeta_{n}\right) \log \left|\tilde{z}_{0}\right|-\left|\Im\left(\zeta_{n}\right)\right|\left|\theta\left(\tilde{z}_{0}\right)\right| \geq-\frac{1}{2} \Re\left(\zeta_{n}\right) \log \left|\tilde{z}_{0}\right| .
$$

The triangle inequality and (290) yield from (298) and (347),

$$
\begin{aligned}
\left|\tilde{z}_{0}^{-\zeta_{n}}-1\right| \geq|| \tilde{z}_{0}^{-\zeta_{n}}|-1|=\left|e^{g\left(\zeta_{n}, \tilde{z}_{0}\right)}-1\right| & \geq \frac{1}{e} \min \left\{1,\left|g\left(\zeta_{n}, \tilde{z}_{0}\right)\right|\right\} \\
& \geq \frac{1}{e} \min \left\{1,-\frac{1}{2} \Re\left(\zeta_{n}\right) \log \left|\tilde{z}_{0}\right|\right\} \\
& \geq-c \alpha^{2} \log |\alpha|,
\end{aligned}
$$

where we have used $\left|\tilde{z}_{0}\right| \leq c_{2} \alpha^{2}$. Hence, we have for $|\alpha| \ll 1$ and $\left|z_{0}\right| \leq \alpha^{2}$,

$$
\left|\left(\tilde{z}_{0}^{-\zeta_{n}}-1\right) \frac{1}{\tilde{z}_{0}}\right| \geq \frac{-c \alpha^{2} \log |\alpha|}{c_{2}|\alpha|^{2}}=-\frac{c}{c_{2}} \log |\alpha|,
$$

which goes to $\infty$ as $\alpha \rightarrow 0$, and we have arrived at the contradiction with (288) if $|\alpha|$ is small enough. Next we consider the case (2) of (ii). In this case we simply compute

$$
\left.\left|g\left(\zeta_{n}, \tilde{z}_{0}\right)\right| \geq \kappa\left|\Im\left(\zeta_{n}\right)\right|\left|\theta\left(\tilde{z}_{0}\right)\right| \geq-\frac{\kappa}{2} \Re\left(\zeta_{n}\right) \log \mid \tilde{z}_{0}\right) \mid .
$$

Then, exactly as in the case (1), we can derive the lower bound

$$
\left|\left(\tilde{z}_{0}^{-\zeta_{n}}-1\right) \frac{1}{\tilde{z}_{0}}\right| \geq-\frac{c \kappa}{c_{2}} \log |\alpha|,
$$

which contradicts with (288) for $|\alpha| \ll 1$. Collecting these, we conclude that there are no zero points of $F_{n}(z ; \alpha)$ with $|n|=1$ in $\Sigma_{\frac{\pi}{2}-\epsilon} \cap B_{\alpha^{2}}(0)$ if $|\alpha| \leq \delta_{\epsilon} \ll 1$. The proof is complete.

Proof of Theorem 1.5 (4). Fix arbitrary $\epsilon \in\left(0, \frac{\pi}{2}\right)$. Since $|\alpha|$ is assumed to be small, by Theorem 1.6 it suffices to show that there are no zero points of $F_{n}(\sqrt{\lambda} ; \alpha)$ in $\bar{\Sigma}_{\pi-\epsilon} \backslash\{0\}$ for $|n|=1$. Let $\delta_{\epsilon / 3} \in(0,1)$ be the number in Proposition 3.34. If $|\alpha| \leq \delta_{\epsilon / 3}$ then $F_{n}(\sqrt{\lambda} ; \alpha) \neq 0$ for any $\lambda \in \bar{\Sigma}_{\pi-\epsilon} \backslash\{0\}$ with $|\lambda| \leq \alpha^{4}$, since we have $\sqrt{\lambda} \in \Sigma_{\frac{\pi}{2}-\frac{\epsilon}{3}}$ and $|\sqrt{\lambda}| \leq \alpha^{2}$ in this case. On the other hand, Corollary 3.28 implies that the set

$$
\begin{aligned}
D(\alpha) & :=\left\{\lambda \in \mathbb{C}|| \Im(\lambda) \mid>-\kappa \Re(\lambda)+\kappa \alpha^{2} e^{-\frac{c_{\kappa}}{|\alpha|}}\right\}, \\
\kappa & =\frac{1}{2} \min \left\{|\tan (\pi-\epsilon)|, \frac{1}{2}\right\},
\end{aligned}
$$

is included in the resolvent set $\rho\left(-\left.A_{\alpha}\right|_{\mathcal{P}_{n} L_{\sigma}^{2}}\right)$ for $|n|=1$ and $|\alpha| \leq \tilde{\delta}_{\kappa}$. Hence, by Proposition 3.2 we conclude that $F_{n}(\sqrt{\lambda} ; \alpha) \neq 0$ for all $\lambda \in D(\alpha)$ if $|n|=1$ and $|\alpha| \leq \tilde{\delta}_{\kappa}$. The definition of $D(\alpha)$ in (303) shows that there is $\delta_{\epsilon}^{\prime} \in\left(0, \delta_{\epsilon / 3}\right]$ such that if $|\alpha| \leq \delta_{\epsilon}^{\prime}$ then

$$
\bar{\Sigma}_{\pi-\epsilon} \backslash\{0\} \subset D(\alpha) \cup B_{\alpha^{4}}(0) .
$$

Therefore, $F_{n}(\sqrt{\lambda} ; \alpha) \neq 0$ for $\lambda \in \bar{\Sigma}_{\pi-\epsilon} \backslash\{0\}$ if $|\alpha| \leq \delta_{\epsilon}^{\prime}$ and $|n|=1$. The proof is complete.

Proof of Theorem 1.3. In the proof of Theorem 1.5 (4) above we have just proved that the assumption (15) of Theorem 1.6 holds for sufficiently small $|\alpha|$. Hence Theorem 1.3 is a direct consequence of Theorem 1.6. The proof is complete. 


\section{Nonlinear stability for the case $|\alpha| \ll 1$}

In this section we will prove Theorem 1.1 by using the estimates for the perturbed Stokes semigroup obtained in Theorem 1.3.

Proof of Theorem 1.1. (Existence) Firstly let us assume that $v_{0} \in L_{\sigma}^{2}(\Omega) \cap L^{q}(\Omega)^{2}$ for some $q \in\left[\frac{3}{2}, 2\right]$. We will solve $\left(\mathrm{INS}_{\alpha}\right)$ by applying the contraction mapping theorem. To this end we introduce the space-time norm

$$
\|v\|_{X, q}=\sum_{k=0,1} \sup _{t>0}(1+t)^{\frac{1}{q}-\frac{1}{2}} t^{\frac{k}{2}}\left\|\nabla^{k} v(t)\right\|_{L^{2}(\Omega)},
$$

and we set

$$
\begin{aligned}
& X_{R_{2}, R_{q}, q}=\left\{v \in C\left([0, \infty) ; L_{\sigma}^{2}(\Omega)\right) \cap C\left((0, \infty) ; W_{0}^{1,2}(\Omega)^{2}\right) \mid\right. \\
&\left.\lim _{t \rightarrow 0} t^{\frac{1}{2}}\|\nabla v(t)\|_{L^{2}(\Omega)}=0, \quad\|v\|_{X, 2} \leq R_{2}, \quad\|v\|_{X, q} \leq R_{q}\right\}
\end{aligned}
$$

for fixed $R_{2}, R_{q}>0$. We also set for $v \in X_{R_{2}, R_{q}, q}$,

$$
\begin{aligned}
H\left[v ; v_{0}\right](t) & =e^{-t A_{\alpha}} v_{0}-\int_{0}^{t} e^{-(t-s) A_{\alpha}} \mathbb{P}(v(s) \cdot \nabla v(s)) \mathrm{d} s, \\
H_{0}\left[v_{0}\right](t) & =H\left[0 ; v_{0}\right](t)=e^{-t A_{\alpha}} v_{0} .
\end{aligned}
$$

By Theorem 1.3 we have

$$
\left\|H_{0}\left[v_{0}\right]\right\|_{X, q} \leq C\left(\left\|v_{0}\right\|_{L^{2}(\Omega)}+\left\|v_{0}\right\|_{L^{q}(\Omega)}\right),
$$

where the constant $C$ depends only on $\alpha$ and $q$. Moreover, since $D_{L_{\sigma}^{2}}\left(A_{\alpha}\right)$ is dense in $L_{\sigma}^{2}(\Omega)$ and

$$
\left\|e^{-t A_{\alpha}} f\right\|_{W_{0}^{1,2}(\Omega)} \leq C\left\|e^{-t A_{\alpha}} f\right\|_{D_{L_{\sigma}^{2}}\left(A_{\alpha}\right)} \leq C\|f\|_{D_{L_{\sigma}^{2}}\left(A_{\alpha}\right)},
$$

we have from (7) that

$$
\lim _{t \rightarrow 0} t^{\frac{1}{2}}\left\|\nabla e^{-t A_{\alpha}} v_{0}\right\|_{L^{2}(\Omega)}=0, \quad v_{0} \in L_{\sigma}^{2}(\Omega) .
$$

Next we estimate the nonlinear term. Let $v, w \in X_{R_{2}, R_{q}, q}$. Then for $k=0,1$ we have from Theorem 1.3,

$$
\begin{aligned}
& \left\|\nabla^{k} \int_{0}^{t} e^{-(t-s) A_{\alpha}} \mathbb{P}(v(s) \cdot \nabla w(s)) \mathrm{d} s\right\|_{L^{2}(\Omega)} \\
& \leq C \int_{0}^{t}(t-s)^{-\frac{1}{4}-\frac{k}{2}}\|\mathbb{P}(v(s) \cdot \nabla w(s))\|_{L^{\frac{4}{3}(\Omega)}} \mathrm{d} s .
\end{aligned}
$$

We recall that the Helmholtz projection is bounded also from $L^{p}(\Omega)^{2}$ to $L_{\sigma}^{p}(\Omega)$ for $1<p<$ $\infty$; [43]. Then the Hölder inequality and the Gagliardo-Nirenberg inequality yield

$$
\begin{aligned}
\|\mathbb{P}(v(s) \cdot \nabla w(s))\|_{L^{\frac{4}{3}}(\Omega)} & \leq C\|v(s)\|_{L^{4}(\Omega)}\|\nabla w(s)\|_{L^{2}(\Omega)} \\
& \leq C\|v(s)\|_{L^{2}(\Omega)}^{\frac{1}{2}}\|\nabla v(s)\|_{L^{2}(\Omega)}^{\frac{1}{2}}\|\nabla w(s)\|_{L^{2}(\Omega)},
\end{aligned}
$$


and thus, by the definition of the norm $\|\cdot\|_{X, q}$ we have

$$
\begin{aligned}
& \left\|\nabla^{k} \int_{0}^{t} e^{-(t-s) A_{\alpha}} \mathbb{P}(v(s) \cdot \nabla w(s)) \mathrm{d} s\right\|_{L^{2}(\Omega)} \\
& \leq C \int_{0}^{t}(t-s)^{-\frac{1}{4}-\frac{k}{2}} s^{-\frac{3}{4}}(1+s)^{-\left(\frac{1}{q}-\frac{1}{2}\right)} \mathrm{d} s\|v\|_{X, 2}\|w\|_{X, q} .
\end{aligned}
$$

Then we see

$$
\int_{0}^{t}(t-s)^{-\frac{1}{4}-\frac{k}{2}} s^{-\frac{3}{4}}(1+s)^{-\left(\frac{1}{q}-\frac{1}{2}\right)} \mathrm{d} s \leq C t^{-\frac{k}{2}}
$$

while for $t>1$,

$$
\begin{aligned}
\int_{0}^{t}(t-s)^{-\frac{1}{4}-\frac{k}{2}} s^{-\frac{3}{4}}(1+s)^{-\left(\frac{1}{q}-\frac{1}{2}\right)} \mathrm{d} s \leq & C t^{-\frac{1}{4}-\frac{k}{2}} \int_{0}^{\frac{t}{2}} s^{-\frac{3}{4}}(1+s)^{-\left(\frac{1}{q}-\frac{1}{2}\right)} \mathrm{d} s \\
& +C(1+t)^{-\left(\frac{1}{q}-\frac{1}{2}\right)} \int_{\frac{t}{2}}^{t}(t-s)^{-\frac{1}{4}-\frac{k}{2}} s^{-\frac{3}{4}} \mathrm{~d} s \\
\leq & C t^{-\frac{k}{2}-\frac{1}{q}+\frac{1}{2}}
\end{aligned}
$$

Hence we obtain

$$
\begin{aligned}
& \sum_{k=0,1} \sup _{t>0}(1+t)^{\frac{1}{q}-\frac{1}{2}} t^{\frac{k}{2}}\left\|\nabla^{k} \int_{0}^{t} e^{-(t-s) A_{\alpha}} \mathbb{P}(v(s) \cdot \nabla w(s)) \mathrm{d} s\right\|_{L^{2}(\Omega)} \\
& \quad \leq C_{0}\|v\|_{X, 2}\|w\|_{X, q}
\end{aligned}
$$

Here the constant $C_{0}$ is taken depending only on $\alpha$ if $q \in\left[\frac{3}{2}, 2\right]$. On the other hand, from (311) with $k=1$ and (312) we also have

$$
\begin{aligned}
& \limsup _{t \rightarrow 0} t^{\frac{1}{2}}\left\|\nabla \int_{0}^{t} e^{-(t-s) A_{\alpha}} \mathbb{P}(v(s) \cdot \nabla w(s)) \mathrm{d} s\right\|_{L^{2}(\Omega)} \\
& \leq C\|v\|_{X, 2} \limsup _{t \rightarrow 0} \sup _{0<s<t} s^{\frac{1}{2}}\|\nabla w(s)\|_{L^{2}(\Omega)}=0,
\end{aligned}
$$

which implies, together with (310), that

$$
\lim _{t \rightarrow 0} t^{\frac{1}{2}}\left\|\nabla H\left[v ; v_{0}\right](t)\right\|_{L^{2}(\Omega)}=0, \quad v \in X_{R_{2}, R_{q}, q}, \quad v_{0} \in L_{\sigma}^{2}(\Omega) .
$$

By using Theorem 1.3 it is not difficult to see that $H\left[v ; v_{0}\right]$ belongs to $C\left([0, \infty) ; L_{\sigma}^{2}(\Omega)\right)$ and to $C\left((0, \infty) ; W_{0}^{1,2}(\Omega)^{2}\right)$ for all $v \in X_{R_{2}, R_{q}, q}$ and $v_{0} \in L_{\sigma}^{2}(\Omega)$, where the behavior $\lim _{t \rightarrow 0} t^{1 / 2}\|\nabla v(t)\|_{L^{2}(\Omega)}=0$ has to be used for the nonlinear term in order to show the continuity in $L_{\sigma}^{2}(\Omega)$ at $t=0$. This argument is standard and thus the details are omitted here. Now let us take $R_{2}=2\left\|H\left[0 ; v_{0}\right]\right\|_{X, 2}$ and $R_{q}=2\left\|H\left[0 ; v_{0}\right]\right\|_{X, q}$. By (309) if $\left\|v_{0}\right\|_{L^{2}(\Omega)}$ is small enough then $C_{0} R_{2} \leq \frac{1}{8}$ holds, where $C_{0}$ is the number in (314). Then, collecting (310), (314), and (316), we have proved that $H\left[\cdot ; v_{0}\right]$ is a mapping from $X_{R_{2}, R_{q}, q}$ to $X_{R_{2}, R_{q}, q}$. Furthermore, since the nonlinear term is a bilinear form, the estimate (314) and the choice of $R_{2}$ imply that $H\left[\cdot ; v_{0}\right]$ is a contraction mapping on $X_{R_{2}, R_{q}, q}$. Hence there exists a unique $v \in X_{R_{2}, R_{q}, q}$ such that $v(t)=H\left[v ; v_{0}\right](t)$ for $t \geq 0$, which is the solution to $\left(\mathrm{INS}_{\alpha}\right)$. Note that the smallness is required only for $\left\|v_{0}\right\|_{L^{2}(\Omega)}$ here. By considering the 
case $q=2$ above, we have shown that for any $v_{0} \in L_{\sigma}^{2}(\Omega)$ with $\left\|v_{0}\right\|_{L^{2}(\Omega)} \ll 1$ there exists a unique solution $v \in C\left([0, \infty) ; L_{\sigma}^{2}(\Omega)\right)$ to $\left(\operatorname{INS}_{\alpha}\right)$ satisfying

$$
\sup _{t \geq 0}\|v(t)\|_{L^{2}(\Omega)}+\sup _{t>0} t^{\frac{1}{2}}\|\nabla v(t)\|_{L^{2}(\Omega)} \leq C\left\|v_{0}\right\|_{L^{2}(\Omega)} \ll 1 .
$$

Finally let us prove (6) for this solution $v$. For each $N \gg 1$ let us take $v_{0, N} \in C_{0, \sigma}^{\infty}(\Omega)$ so that $\left\|v_{0}-v_{0, N}\right\|_{L^{2}(\Omega)} \leq N^{-1}$. Since $\left\|v_{0}^{(N)}\right\|_{L^{2}(\Omega)}$ is also sufficiently small for sufficiently large $N$, the above argument shows that we can find the unique solution $v^{(N)} \in$ $X_{R_{2}^{(N)}, R_{3 / 2}^{(N)}, 3 / 2}$ to $\left(\mathrm{INS}_{\alpha}\right)$ with $v_{0}$ replaced by $v_{0, N}$, where $R_{2}^{(N)}=2\left\|H\left[0 ; v_{0}^{(N)}\right]\right\|_{X, 2} \leq \frac{1}{4 C_{0}}$ and $R_{3 / 2}^{(N)}=2\left\|H\left[0 ; v_{0}^{(N)}\right]\right\|_{X, 3 / 2}$. Then (309) and the bilinear estimate (314) with $q=2$ imply that

$$
\begin{aligned}
\left\|v-v^{(N)}\right\|_{X, 2} & \leq\left\|H\left[0 ; v_{0}\right]-H\left[0 ; v_{0, N}\right]\right\|_{X, 2}+\left\|H[v ; 0]-H\left[v^{(N)} ; 0\right]\right\|_{X, 2} \\
& \leq C\left\|v_{0}-v_{0, N}\right\|_{L^{2}(\Omega)}+C_{0}\left(R_{2}+R_{2}^{(N)}\right)\left\|v-v^{(N)}\right\|_{X, 2} \\
& \leq \frac{C}{N}+\frac{1}{2}\left\|v-v^{(N)}\right\|_{X, 2}
\end{aligned}
$$

and hence,

$$
\left\|v(t)-v^{(N)}(t)\right\|_{L^{2}(\Omega)}+t^{\frac{1}{2}}\left\|\nabla\left(v(t)-v^{(N)}(t)\right)\right\|_{L^{2}(\Omega)} \leq \frac{2 C}{N}, \quad t>0 .
$$

On the other hand, since $v^{(N)} \in X_{R_{2}^{(N)}, R_{3 / 2}^{(N)}, 3 / 2}$ we have

$$
\lim _{t \rightarrow \infty}\left(\left\|v^{(N)}(t)\right\|_{L^{2}(\Omega)}+t^{\frac{1}{2}}\left\|\nabla v^{(N)}(t)\right\|_{L^{2}(\Omega)}\right)=0 .
$$

Combining (318) with (319), we obtain (6) by taking $N \rightarrow \infty$ after $t \rightarrow \infty$.

(Uniqueness) Let $w \in C\left([0, \infty) ; L_{\sigma}^{2}(\Omega)\right)$ be another solution to $\left(\mathrm{INS}_{\alpha}\right)$ satisfying $t^{1 / 2} \nabla w(t) \in$ $L^{\infty}\left(0, \infty ; L^{2}(\Omega)\right)$. It suffices to show $v(t)=w(t)$ for $t \in\left(0, T_{0}\right]$ with $T_{0} \ll 1$, since we can repeat the same argument for $t>T_{0}$.

Step 1. $\lim _{t \rightarrow 0}\|H[w ; 0](t)\|_{L^{2}(\Omega)}=0$ : To prove this we observe that $\left\{e^{-t A_{\alpha}}\right\}_{t \geq 0}$ defines a $C_{0}$-analytic semigroup in $L_{\sigma}^{p}(\Omega)$ for any $p \in(1, \infty)$ as the perturbation of the Stokes semigroup $\left\{e^{-t A}\right\}_{t \geq 0}$ in $L_{\sigma}^{p}(\Omega)$. This is easily shown by using the regularity $U \in L^{\infty}(\Omega)^{2}$, and the details are omitted here. Using this fact one can show that $H[w ; 0](t)$ converges to 0 weakly in $L_{\sigma}^{2}(\Omega)$ by the density argument. On the other hand, since $e^{-t A_{\alpha}} v_{0}$ converges to $v_{0}$ in $L_{\sigma}^{2}(\Omega)$ as $t \rightarrow 0$, the assumption $w \in C\left([0, \infty) ; L_{\sigma}^{2}(\Omega)\right)$ implies that $H[w ; 0](t)=$ $w(t)-e^{-t A_{\alpha}} v_{0}$ actually converges to 0 strongly in $L_{\sigma}^{2}(\Omega)$ as $t \rightarrow 0$. Thus we have proved the claim.

Step 2. $\lim _{t \rightarrow 0} t^{1 / 4}\|w(t)\|_{L^{4}(\Omega)}=0$ : From the integral equation and the regularity assumption of

$$
w \in C\left([0, \infty) ; L_{\sigma}^{2}(\Omega)\right), \quad t^{1 / 2} \nabla w(t) \in L^{\infty}\left(0, \infty ; L^{2}(\Omega)\right),
$$

we can show as in (314) that

$$
H[w ; 0](t) \in W_{0}^{1,2}(\Omega)^{2}, \quad t>0, \quad t^{1 / 2} \nabla H[w ; 0](t) \in L^{\infty}\left(0,1 ; L^{2}(\Omega)\right) .
$$


Then, by the interpolation and Step 1 we have

$$
\begin{aligned}
t^{\frac{1}{4}}\|H[w ; 0](t)\|_{L^{4}(\Omega)} & \leq C\|H[w ; 0](t)\|_{L^{2}(\Omega)}^{\frac{1}{2}}\left(t^{\frac{1}{2}}\|\nabla H[w ; 0](t)\|_{L^{2}(\Omega)}\right)^{\frac{1}{2}} \\
& \rightarrow 0 \quad(t \rightarrow 0) .
\end{aligned}
$$

On the other hand, as in (310), it is straightforward to see $\lim _{t \rightarrow 0} t^{1 / 4}\left\|e^{-t A_{\alpha}} v_{0}\right\|_{L^{4}(\Omega)}=0$, which shows the claim by (320).

Step 3. $v(t)=w(t)$ : Now let us compute the difference $z(t)=v(t)-w(t)$, where $v(t)$ is the solution to $\left(\mathrm{INS}_{\alpha}\right)$ constructed in the existence proof above. In particular, $v$ satisfies $\lim _{t \rightarrow 0} t^{1 / 2}\|\nabla v(t)\|_{L^{2}(\Omega)}=0$. Then $z(t)$ satisfies the integral equation

$$
z(t)=-\int_{0}^{t} e^{-(t-s) A_{\alpha}} \mathbb{P}(z(s) \cdot \nabla v(s)) \mathrm{d} s-\int_{0}^{t} e^{-(t-s) A_{\alpha}} \mathbb{P}(w(s) \cdot \nabla z(s)) \mathrm{d} s .
$$

Thus, by using Theorem 1.3 as above and also using the inequality of the form

$$
\|\mathbb{P}(f \cdot \nabla g)\|_{L^{\frac{4}{3}(\Omega)}} \leq C\|f\|_{L^{4}(\Omega)}\|\nabla g\|_{L^{2}(\Omega)},
$$

we have for $t \in\left(0, T_{0}\right]$,

$$
\begin{aligned}
t^{\frac{1}{4}}\|z(t)\|_{L^{4}(\Omega)}+t^{\frac{1}{2}}\|\nabla z(t)\|_{L^{2}(\Omega)} \leq & C \sup _{0<t<T_{0}} t^{\frac{1}{2}}\|\nabla v(t)\|_{L^{2}(\Omega)} \sup _{0<t<T_{0}} t^{\frac{1}{4}}\|z(t)\|_{L^{4}(\Omega)} \\
& +C \sup _{0<t<T_{0}} t^{\frac{1}{4}}\|w(t)\|_{L^{4}(\Omega)} \sup _{0<t<T_{0}} t^{\frac{1}{2}}\|\nabla z(t)\|_{L^{2}(\Omega)} .
\end{aligned}
$$

Hence, if $T_{0}$ is small enough we conclude that $z(t)=0$ in $\left(0, T_{0}\right]$, as desired. The proof of Theorem 1.1 is complete.

\section{A Formula of modified Bessel functions}

In this appendix we collect some basic results on the modified Bessel functions of second kind. Let $I_{\mu}(z)$ and $K_{\mu}(z)$ be the modified Bessel functions of order $\mu$ defined as (52) and (10), respectively.

Lemma A.1 It follows that

$$
\begin{aligned}
2 \frac{\mathrm{d} I_{\mu}}{\mathrm{d} z}(z) & =I_{\mu-1}(z)+I_{\mu+1}(z), \\
\frac{2 \mu}{z} I_{\mu}(z) & =I_{\mu-1}(z)-I_{\mu+1}(z), \\
\frac{\mathrm{d} I_{\mu}}{\mathrm{d} z}(z) & =-\frac{\mu}{z} I_{\mu}(z)+I_{\mu-1}(z)=\frac{\mu}{z} I_{\mu}(z)+I_{\mu+1}(z),
\end{aligned}
$$

and that

$$
\begin{aligned}
2 \frac{\mathrm{d} K_{\mu}}{\mathrm{d} z}(z) & =-K_{\mu-1}(z)-K_{\mu+1}(z), \\
\frac{2 \mu}{z} K_{\mu}(z) & =-K_{\mu-1}(z)+K_{\mu+1}(z), \\
\frac{\mathrm{d} K_{\mu}}{\mathrm{d} z}(z) & =-\frac{\mu}{z} K_{\mu}(z)-K_{\mu-1}(z)=\frac{\mu}{z} K_{\mu}(z)-K_{\mu+1}(z) .
\end{aligned}
$$


Proof. These are well known; see, e.g., [1, Chapter 4]. We note that (324) follows from (322) and (323), and similarly, (325) follows from (326) and (327). The details are omitted here.

Lemma A.2 Let $\Re(\mu)>0$. Then for any $M>0$ there is a constant $C=C(M, \mu)>0$ such that if $|z| \leq M$ then

$$
\left|I_{\mu}(z)\right| \leq C|z|^{\Re(\mu)}, \quad\left|K_{\mu}(z)\right| \leq C|z|^{-\Re(\mu)} .
$$

Proof. The assertion immediately follows from the definition of $I_{\mu}$ in (52) and the relation (53), including the case $\mu=1,2, \cdots$. The proof is complete.

Lemma A.3 Let $\epsilon \in\left(0, \frac{\pi}{2}\right)$. Then there is a constant $C=C(\epsilon, \mu)>0$ such that if $z \in \Sigma_{\frac{\pi}{2}-\epsilon}$ and $|z| \geq 1$ then

$$
\left|I_{\mu}(z)\right| \leq C|\Re(z)|^{-\frac{1}{2}} e^{\Re(z)}, \quad\left|K_{\mu}(z)\right| \leq C|\Re(z)|^{-\frac{1}{2}} e^{-\Re(z)} .
$$

Moreover, for $|z| \gg 1$ the following expansion holds.

$$
\begin{aligned}
& I_{\mu}(z)=\frac{1}{\sqrt{2 \pi z}} e^{z}\left(1+l_{\mu}(z)\right), \quad K_{\mu}(z)=\sqrt{\frac{\pi}{2 z}} e^{-z}\left(1+k_{\mu}(z)\right), \\
& \left|l_{\mu}(z)\right|+\left|k_{\mu}(z)\right| \leq C|z|^{-1} .
\end{aligned}
$$

Proof. The estimate (329) follows from (328) and (330), while (330) is well known; see [1, Chapter 4.12]. The proof is complete.

Lemma A.4 Let $k=0,1$ and $\Re(\mu)>|n|$, and let $\epsilon \in\left(0, \frac{\pi}{2}\right)$. Then there is a constant $C=C(\epsilon, \mu, n)>0$ such that the following statements hold.

(1) If $\lambda \in \Sigma_{\pi-\epsilon}$ and $\Re(\sqrt{\lambda}) \geq \tau^{-1}$ then

$$
\int_{\tau}^{r} s^{1-k+|n|}\left|K_{\mu-k}(\sqrt{\lambda} s)\right| \mathrm{d} s \leq C|\lambda|^{-\frac{3}{4}} \tau^{\frac{1}{2}-k+|n|} e^{-\tau \Re(\sqrt{\lambda})} .
$$

(2) If $\lambda \in \Sigma_{\pi-\epsilon}$ and $r^{-1} \leq \Re(\sqrt{\lambda}) \leq \tau^{-1}$ then

$$
\int_{\tau}^{r} s^{1-k+|n|}\left|K_{\mu-k}(\sqrt{\lambda} s)\right| \mathrm{d} s \leq C\left(|\lambda|^{-1-\frac{|n|-k}{2}}+|\lambda|^{-\frac{\Re(\mu)-k}{2}-1} \tau^{n \mid-\Re(\mu)}\right) .
$$

(3) If $\lambda \in \Sigma_{\pi-\epsilon}$ and $\Re(\sqrt{\lambda}) \leq r^{-1}$ then

$$
\int_{\tau}^{r} s^{1-k+|n|}\left|K_{\mu-k}(\sqrt{\lambda} s)\right| \mathrm{d} s \leq C|\lambda|^{-\frac{\Re(\mu)-k}{2}} r^{2} \tau^{|n|-\Re(\mu)} .
$$

(4) If $\lambda \in \Sigma_{\pi-\epsilon}$ and $\Re(\sqrt{\lambda}) \geq r^{-1}$ then

$$
\int_{r}^{\infty} s^{1-k-|n|}\left|K_{\mu-k}(\sqrt{\lambda} s)\right| \mathrm{d} s \leq C|\lambda|^{-\frac{3}{4}} r^{\frac{1}{2}-k-|n|} e^{-r \Re(\sqrt{\lambda})} .
$$

(5) If $\lambda \in \Sigma_{\pi-\epsilon}$ and $\Re(\sqrt{\lambda}) \leq r^{-1}$ then

$$
\int_{r}^{\infty} s^{1-k-|n|}\left|K_{\mu-k}(\sqrt{\lambda} s)\right| \mathrm{d} s \leq C|\lambda|^{-\frac{\Re(\mu)-k}{2}} r^{2-|n|-\Re(\mu)} .
$$


Proof. (1) We have from Lemma A.3,

$$
\begin{aligned}
\int_{\tau}^{r} s^{1-k+|n|}\left|K_{\mu-k}(\sqrt{\lambda} s)\right| \mathrm{d} s & \leq C|\lambda|^{-\frac{1}{4}} \int_{\tau}^{\infty} s^{\frac{1}{2}-k+|n|} e^{-s \Re(\sqrt{\lambda})} \mathrm{d} s \\
& \leq C|\lambda|^{-\frac{3}{4}} \tau^{\frac{1}{2}-k+|n|} e^{-\tau \Re(\sqrt{\lambda})} .
\end{aligned}
$$

(2) We divide the integral into $\int_{\tau}^{\frac{1}{\Re(\sqrt{\lambda})}}$ and $\int_{\frac{1}{\Re(\sqrt{\lambda})}}^{r}$, and the second term is bounded from above by $C|\lambda|^{-1-\frac{|n|-k}{2}}$, due to (331). On the other hand, if $\lambda \in \Sigma_{\pi-\epsilon}$ and $s \Re(\sqrt{\lambda}) \leq 1$ then $|s \sqrt{\lambda}| \leq C$, where $C$ depends only on $\epsilon$. Hence, by using Lemma A.2 the first term is estimated as

$$
\begin{aligned}
\int_{\tau}^{\frac{1}{\Re(\sqrt{\lambda})}} s^{1-k+|n|}\left|K_{\mu-k}(\sqrt{\lambda} s)\right| \mathrm{d} s & \leq C \int_{\tau}^{\frac{1}{\Re(\sqrt{\lambda})}} s^{1-k+|n|}|\sqrt{\lambda} s|^{-\Re(\mu)+k} \mathrm{~d} s \\
& \leq C|\lambda|^{-\frac{\Re(\mu)-k}{2}} \int_{\tau}^{\frac{1}{\Re(\sqrt{\lambda})}} s^{1+|n|-\Re(\mu)} \mathrm{d} s \\
& \leq C|\lambda|^{-\frac{\Re(\mu)-k}{2}-1} \tau^{|n|-\Re(\mu)} .
\end{aligned}
$$

Here we have used the condition $\Re(\mu)>|n|$.

(3) The proof is very similar to (332), where Lemma A.2 is used. We omit the details.

(4) We have from Lemma A.3,

$$
\begin{aligned}
\int_{r}^{\infty} s^{1-k-|n|}\left|K_{\mu-k}(\sqrt{\lambda} s)\right| \mathrm{d} s C & \leq C|\lambda|^{-\frac{1}{4}} \int_{r}^{\infty} s^{\frac{1}{2}-k-|n|} e^{-s \Re(\sqrt{\lambda})} \mathrm{d} s \\
& \leq C|\lambda|^{-\frac{3}{4}} r^{\frac{1}{2}-k-|n|} e^{-r \Re(\sqrt{\lambda})} .
\end{aligned}
$$

(5) We divide the integral into $\int_{r}^{\frac{1}{\Re(\sqrt{\lambda})}}$ and $\int_{\frac{1}{\Re(\sqrt{\lambda})}}^{\infty}$, and the second term is bounded from above by $C|\lambda|^{\frac{|n|+k-1}{2}-\frac{1}{2}}$, due to (334). Since $\Re(\mu)>|n| \geq 1$ and $\Re(\sqrt{\lambda}) \leq r^{-1}$ we have $|\lambda|^{\frac{|n|+k-1}{2}-\frac{1}{2}}=|\lambda|^{-\frac{\Re(\mu)-k}{2}+\frac{\Re(\mu)+|n|-2}{2}} \leq C|\lambda|^{-\frac{\Re(\mu)-k}{2}} r^{-\Re(\mu)-|n|+2}$. Next we estimate the integral $\int_{r}^{\frac{1}{\Re(\sqrt{\lambda})}}$. Lemma A.3 yields

$$
\begin{aligned}
\int_{r}^{\frac{1}{\Re(\sqrt{\lambda})}} s^{1-k-|n|}\left|K_{\mu-k}(\sqrt{\lambda} s)\right| \mathrm{d} s & \leq C \int_{r}^{\frac{1}{\Re(\sqrt{\lambda})}} s^{1-k-|n|}|\sqrt{\lambda} s|^{-\Re(\mu)+k} \mathrm{~d} s \\
& \leq C|\lambda|^{-\frac{\Re(\mu)-k}{2}} r^{2-|n|-\Re(\mu)} .
\end{aligned}
$$

Here we have used the assumption $\Re(\mu)>1$ (for $|n|=1$ ). Collecting these, we obtain (335). The proof is complete.

Lemma A.5 Let $k=0,1$ and $\Re(\mu)>|n|$, and let $\epsilon \in\left(0, \frac{\pi}{2}\right)$. Then there is a constant $C=C(\epsilon, \mu, n)>0$ such that the following statements hold.

(1) If $\lambda \in \Sigma_{\pi-\epsilon}$ and $\Re(\sqrt{\lambda}) \leq \tau^{-1}$ then

$$
\int_{1}^{\tau} s^{1-k+|n|}\left|I_{\mu+k}(\sqrt{\lambda} s)\right| \mathrm{d} s \leq C|\lambda|^{\frac{\Re(\mu)+k}{2}} \tau^{2+|n|+\Re(\mu)} .
$$


(2) If $\lambda \in \Sigma_{\pi-\epsilon}$ and $\Re(\sqrt{\lambda}) \geq \tau^{-1}$ then

$$
\int_{1}^{\tau} s^{1-k+|n|}\left|I_{\mu+k}(\sqrt{\lambda} s)\right| \mathrm{d} s \leq C|\lambda|^{-\frac{3}{4}} \tau^{\frac{1}{2}-k+|n|} e^{\tau \Re(\sqrt{\lambda})} .
$$

(3) If $\lambda \in \Sigma_{\pi-\epsilon}$ and $\Re(\sqrt{\lambda}) \leq \tau^{-1}$ then

$$
\int_{r}^{\tau} s^{1-k-|n|}\left|I_{\mu+k}(\sqrt{\lambda} s)\right| \mathrm{d} s \leq C|\lambda|^{\Re(\mu)+k} \tau^{\Re(\mu)+2-|n|} .
$$

(4) If $\lambda \in \Sigma_{\pi-\epsilon}$ and $\Re(\sqrt{\lambda}) \geq \tau^{-1}$ then

$$
\int_{r}^{\tau} s^{1-k-|n|}\left|I_{\mu+k}(\sqrt{\lambda} s)\right| \mathrm{d} s \leq C\left(|\lambda|^{\frac{|n|+k}{2}-1}+|\lambda|^{-\frac{3}{4}} \tau^{\frac{1}{2}-k-|n|} e^{\tau \Re(\sqrt{\lambda})}\right) .
$$

Proof. (1) We have from Lemma A.2,

$$
\begin{aligned}
\int_{1}^{\tau} s^{1-k+|n|}\left|I_{\mu+k}(\sqrt{\lambda} s)\right| \mathrm{d} s & \leq C \int_{1}^{\tau} s^{1-k+|n|}|\sqrt{\lambda} s|^{\Re(\mu)+k} \mathrm{~d} s \\
& \leq C|\lambda|^{\Re(\mu)+k} \tau^{2+|n|+\Re(\mu)}
\end{aligned}
$$

as desired.

(2) We divide the integral into $\int_{1}^{\max \left\{\frac{1}{\Re(\sqrt{\lambda})}, 1\right\}}$ and $\int_{\max \left\{\frac{1}{\Re(\sqrt{\lambda})}, 1\right\}}^{\tau}$, and the first term is bounded from above by $C|\lambda|^{-\frac{2+|n|-k}{2}}$, due to (336). The second term is bounded from above by using Lemma A.3,

$$
\begin{aligned}
\int_{\max \left\{\frac{1}{\Re(\sqrt{\lambda})}, 1\right\}}^{\tau} s^{1-k+|n|}\left|I_{\mu+k}(\sqrt{\lambda} s)\right| \mathrm{d} s & \leq C|\lambda|^{-\frac{1}{4}} \int_{\frac{1}{\Re(\sqrt{\lambda})}}^{\tau} s^{\frac{1}{2}-k+|n|} e^{s \Re(\sqrt{\lambda})} \mathrm{d} s \\
& \leq C|\lambda|^{-\frac{3}{4}} \tau^{\frac{1}{2}-k+|n|} e^{\tau \Re(\mu)} .
\end{aligned}
$$

(3) As in the proof of (1), we have from Lemma A.2 and $\Re(\mu)>|n|$,

$$
\begin{aligned}
\int_{r}^{\tau} s^{1-k-|n|}\left|I_{\mu+k}(\sqrt{\lambda} s)\right| \mathrm{d} s & \leq C|\lambda|^{\frac{\Re(\mu)+k}{2}} \int_{r}^{\tau} s^{1-|n|+\Re(\mu)} \mathrm{d} s \\
& \leq C|\lambda|^{\frac{\Re(\mu)+k}{2}} \tau^{2+\Re(\mu)-|n|} .
\end{aligned}
$$

(4) We divide the integral into $\int_{r}^{\max \left\{\frac{1}{\Re(\sqrt{\lambda})}, r\right\}}$ and $\int_{\max \left\{\frac{1}{\Re(\sqrt{\lambda})}, r\right\}}^{\tau}$, and the first term is bounded from above by $C|\lambda|^{\frac{|n|+k}{2}-1}$, due to (338). As for the second term, we have from Lemma A.3,

$$
\begin{aligned}
\int_{\max \left\{\frac{1}{\Re(\sqrt{\lambda})}, r\right\}}^{\tau} s^{1-k-|n|}\left|I_{\mu+k}(\sqrt{\lambda} s)\right| \mathrm{d} s & \leq C|\lambda|^{-\frac{1}{4}} \int_{\frac{1}{\Re(\sqrt{\lambda})}}^{\tau} s^{\frac{1}{2}-k-|n|} e^{s \Re(\sqrt{\lambda})} \mathrm{d} s \\
& \leq C|\lambda|^{-1+\frac{k+|n|}{2}} \int_{1}^{\tau \Re(\sqrt{\lambda})} s^{\frac{1}{2}-k-|n|} e^{s} \mathrm{~d} s .
\end{aligned}
$$

For any $m>0$ there is $C=C_{m}>0$ such that $\int_{1}^{N} s^{-m} e^{s} \mathrm{~d} s \leq C_{m} N^{-m} e^{N}$ for all $N>1$. Therefore, the last term is bounded from above by

$$
C|\lambda|^{-1+\frac{k+|n|}{2}}|\tau \Re(\sqrt{\lambda})|^{\frac{1}{2}-k-|n|} e^{\tau \Re(\sqrt{\lambda})}=C|\lambda|^{-\frac{3}{4}} \tau^{\frac{1}{2}-k-|n|} e^{\tau \Re(\sqrt{\lambda})} .
$$

The proof is complete. 
Remark A.6 A similar argument as in the proof of Lemma A.4 and Lemma A.5 yields the following estimates for $\Re(\mu)>1$ and $k=0,1$.

(1) If $\lambda \in \Sigma_{\pi-\epsilon}$ and $\Re(\sqrt{\lambda}) \geq r^{-1}$ then

$$
\int_{r}^{\infty} s\left|K_{\mu-k}(\sqrt{\lambda} s)\right| \mathrm{d} s \leq C|\lambda|^{-\frac{3}{4}} r^{\frac{1}{2}} e^{-r \Re(\sqrt{\lambda})} .
$$

(2) If $\lambda \in \Sigma_{\pi-\epsilon}$ and $\Re(\sqrt{\lambda}) \leq r^{-1}$ then

$$
\int_{r}^{\infty} s\left|K_{\mu-k}(\sqrt{\lambda} s)\right| \mathrm{d} s \leq C\left(|\lambda|^{-1}+|\lambda|^{-\frac{\Re(\mu)+1}{2}} r^{1-\Re(\mu)}\right) .
$$

(3) If $\lambda \in \Sigma_{\pi-\epsilon}$ and $\Re(\sqrt{\lambda}) \leq r^{-1}$ then

$$
\int_{1}^{r}\left|I_{\mu}(\sqrt{\lambda} s)\right| s^{k} \mathrm{~d} s \leq C|\lambda|^{\Re(\mu)} r^{1+k+\Re(\mu)} .
$$

(4) If $\lambda \in \Sigma_{\pi-\epsilon}$ and $\Re(\sqrt{\lambda}) \geq r^{-1}$ then

$$
\int_{1}^{r}\left|I_{\mu}(\sqrt{\lambda} s)\right| s^{k} \mathrm{~d} s \leq C r^{k}\left(|\lambda|^{-\frac{1}{2}}+|\lambda|^{-\frac{3}{4}} r^{-\frac{1}{2}} e^{r \Re(\sqrt{\lambda})}\right) .
$$

Lemma A.7 Let $F_{n, \mu}$ be the function defined as

$$
F_{n, \mu}(z)=\int_{1}^{\infty} s^{1-|n|} K_{\mu}(s z) \mathrm{d} s, \quad \Re(z)>0, \quad \mu \neq 0 .
$$

Then it follows that

$$
(\mu+|n|-2) F_{n, \mu}(z)=K_{\mu}(z)-z \int_{1}^{\infty} s^{2-|n|} K_{\mu-1}(s z) \mathrm{d} s .
$$

Proof. The identity (327) implies

$$
K_{\mu}(s z)=-\frac{s z}{\mu} \frac{\mathrm{d} K_{\mu}}{\mathrm{d} z}(s z)-\frac{s z}{\mu} K_{\mu-1}(s z)=-\frac{s}{\mu} \frac{\mathrm{d}}{\mathrm{d} s} K_{\mu}(s z)-\frac{s z}{\mu} K_{\mu-1}(s z) .
$$

Hence, we have from the integration by parts,

$$
\begin{aligned}
& F_{n, \mu}(z) \\
& =\frac{1}{\mu} K_{\mu}(z)+\frac{2-|n|}{\mu} \int_{1}^{\infty} s^{1-|n|} K_{\mu}(s z) \mathrm{d} s-\frac{z}{\mu} \int_{1}^{\infty} s^{2-|n|} K_{\mu-1}(s z) \mathrm{d} s \\
& =\frac{1}{\mu} K_{\mu}(z)+\frac{2-|n|}{\mu} F_{n, \mu}(z)-\frac{z}{\mu} \int_{1}^{\infty} s^{2-|n|} K_{\mu-1}(s z) \mathrm{d} s
\end{aligned}
$$

which gives (345). The proof is complete.

Corollary A.8 Let $F_{n}$ be the function defined as (12). Let $|n|=1$. Then

$$
\left(\mu_{n}-1\right) F_{n}(z)=K_{\mu_{n}}(z)-\int_{0}^{\infty} \frac{1}{t^{\mu_{n}-1}\left(t^{2}+1\right)}\left(1+\frac{2 t}{z\left(t^{2}+1\right)}\right) e^{-\frac{z}{2}\left(t+\frac{1}{t}\right)} \mathrm{d} t
$$

Here $\mu_{n}=\mu_{n}(\alpha)$ is defined as (11). 
Proof. By Lemma A.7 with $|n|=1$ we have

$$
\left(\mu_{n}-1\right) F_{n}(z)=K_{\mu_{n}}(z)-z \int_{1}^{\infty} s K_{\mu_{n}-1}(s z) \mathrm{d} s .
$$

Then (10) and the Fubini theorem lead to

$$
\int_{1}^{\infty} s K_{\mu_{n}-1}(s z) \mathrm{d} s=\frac{1}{2} \int_{0}^{\infty} \int_{1}^{\infty} s e^{-\frac{s z}{2}\left(t+\frac{1}{t}\right)} \mathrm{d} s t^{-\mu_{n}} \mathrm{~d} t .
$$

Thus the assertion follows from

$$
\int_{1}^{\infty} s e^{-\frac{s z}{2}\left(t+\frac{1}{t}\right)} \mathrm{d} s=\frac{2 t}{z\left(t^{2}+1\right)}\left(1+\frac{2 t}{z\left(t^{2}+1\right)}\right) e^{-\frac{z}{2}\left(t+\frac{1}{t}\right)} .
$$

The proof is complete.

\section{B Behavior of $\mu_{n}(\alpha)$ for $\left|\frac{\alpha}{n}\right| \ll 1$}

In this appendix we state the asymptotic behavior of $\mu_{n}(\alpha)$ for $\left|\frac{\alpha}{n}\right| \ll 1$.

Lemma B.1 Set $\mu_{n}(\alpha)=\left(n^{2}+i \alpha n\right)^{\frac{1}{2}}$. Then $\mu_{n}(\alpha)$ satisfies the expansion for $\left|\frac{\alpha}{n}\right| \ll 1$ as follows.

$$
\begin{aligned}
\Re\left(\mu_{n}(\alpha)\right) & =|n|\left(1+\frac{\alpha^{2}}{8 n^{2}}+O\left(\left|\frac{\alpha}{n}\right|^{4}\right)\right), \\
\left|\Im\left(\mu_{n}(\alpha)\right)\right| & =\frac{|\alpha|}{2}\left(1+O\left(\left|\frac{\alpha}{n}\right|^{2}\right)\right) .
\end{aligned}
$$

Proof. The assertion easily follows from the representation

$$
\begin{gathered}
\Re\left(\mu_{n}(\alpha)\right)=|n| \sqrt{\frac{1+\sqrt{1+\frac{\alpha^{2}}{n^{2}}}}{2}}, \\
\left|\Im\left(\mu_{n}(\alpha)\right)\right|=|n| \sqrt{\frac{\sqrt{1+\frac{\alpha^{2}}{n^{2}}}-1}{2}} .
\end{gathered}
$$

The details are omitted here. The proof is complete.

Acknowledgements The author is partially supported by the Grant-in-Aid for Young Scientists (B) 25800079.

\section{References}

[1] Andrews, G. E., Askey, R., Roy, R.: Special functions. Encyclopedia of Mathematics and its Applications, 71. Cambridge University Press, Cambridge (1999)

[2] Borchers, W.: Zur Stabilität und Faktorisierungsmethode für die Navier-Stokes Gleichungen inkompressibler viskoser Flüssigkeiten. Habilitationsschrift, Universität Paderborn (1992) 
[3] Borchers, W., Miyakawa, T.: On stability of exterior stationary Navier-Stokes flows. Acta Math. 174, 311-382 (1995)

[4] Dan, W., Shibata, Y.: On the $L_{q}-L_{r}$ estimates of the Stokes semigroup in a twodimensional exterior domain. J. Math. Soc. Japan 51, 181-207 (1999)

[5] Dan, W., Shibata, Y.: Remark on the $L_{q}-L_{\infty}$ estimate of the Stokes semigroup in a 2-dimensional exterior domain. Pacific J. Math. 189, 223-239 (1999)

[6] Farwig, R., Galdi, G. P., Kyed, M.: Asymptotic structure of a Leray solution to the Navier-Stokes flow around a rotating body. Pacific J. Math. 253, 367-382 (2011)

[7] Farwig, R., Hishida, T.: Stationary Navier-Stokes flow around a rotating obstacle. Funkcial. Ekvac. 50, 371-403 (2007)

[8] Farwig, R., Hishida, T. Asymptotic profile of steady Stokes flow around a rotating obstacle. Manuscripta Math. 136, 315-338 (2011)

[9] Farwig, R., Hishida, T.: Leading term at infinity of steady Navier-Stokes flow around a rotating obstacle. Math. Nachr. 284, 2065-2077 (2011)

[10] Farwig, R., Neustupa, J.: On the spectrum of a Stokes-type operator arising from flow around a rotating body. Manuscripta Math. 122, 419-437 (2007)

[11] Farwig, R., Sohr, H.: Weighted $L^{q}$-theory for the Stokes resolvent in exterior domains. J. Math. Soc. Japan. 49, 251-288 (1997)

[12] Finn, R.: On the exterior stationary problem for the Navier-Stokes equations, and associated perturbation problems. Arch. Rational Mech. Anal. 19, 363-406 (1965)

[13] Galdi, G. P.: Steady flow of a Navier-Stokes fluid around a rotating obstacle. J. Elast. 71, 1-31 (2003)

[14] Galdi, G. P.: Stationary Navier-Stokes problem in a two-dimensional exterior domain. Handbook of Differential Equations, Stationary partial differential equations. Vol. I., M. Chipot and P. Quittner, eds., pp. 71-155. North-Holland, Amsterdam (2004)

[15] Galdi, G. P., Silvestre, A. L.: Strong solutions to the Navier-Stokes equations around a rotating obstacle. Arch. Ration. Mech. Anal. 176, 331-350 (2005)

[16] Galdi, G. P., Simader, C. G.: New estimates for the steady-state Stokes problem in exterior domains with applications to the Navier-Stokes problem. Differential Integral Equations. 7, 847-861 (1994)

[17] Gallay, Th., Maekawa, Y.: Long-time asymptotics for two-dimensional exterior flows with small circulation at infinity. Anal. PDE. 6, 973-991 (2013)

[18] Geissert, M., Heck, H., Hieber, M.: $L^{p}$-theory of the Navier-Stokes flowin the exterior of a moving or rotating obstacle. J. Reine Angew. Math. 596, 45-62 (2006)

[19] Hillairet, M., Wittwer, P.: On the existence of solutions to the planar exterior NavierStokes system. J. Differential Equations. 255, 2996-3019 (2013)

[20] Hishida, T.: An existence theorem for the Navier-Stokes flow in the exterior of a rotating obstacle. Arch. Rational Mech. Anal. 150, 307-348 (1999) 
[21] Hishida, T.: $L^{q}$ estimates of weak solutions to the stationary Stokes equations around a rotating body. J. Math. Soc. Japan. 58, 743-767 (2006)

[22] Hishida, T.: Asymptotic structure of steady Stokes flow around a rotating obstacle in two dimensions. Preprint, arXiv:1503.02321.

[23] Hishida, T., Shibata, Y.: $L^{p}-L^{q}$ estimate of the Stokes operator and Navier-Stokes flows in the exterior of a rotating obstacle. Arch. Ration. Mech. Anal. 193, 339-421 (2009)

[24] Hishida, T., Schonbek, M. E.: Stability of time-dependent Navier-Stokes flow and algebraic energy decay. Preprint, arXiv:1412.0204.

[25] Iftimie, D., Karch, G., Lacave, C.: Self-similar asymptotics of solutions to the NavierStokes system in two dimensional exterior domain. Preprint, arXiv:1107.2054.

[26] Iftimie, D., Karch, G., Lacave, C.: Asymptotics of solutions to the Navier-Stokes system in exterior domains. J. Lond. Math. Soc. (2) 90, 785-806 (2014)

[27] Kato, T.: Perturbation Theory for Linear Operators, second edition. Springer-Verlag (1976)

[28] Karch, G., Pilarczyk, D., Schonbek, M. E.: $L^{2}$-asymptotic stability of mild solutions to the Navier-Stokes system of equations in $\mathbb{R}^{3}$. Preprint, arXiv:1308.6667.

[29] Korolev, A., Šverák, V.: On the large-distance asymptotics of steady state solutions of the Navier-Stokes equations in 3D exterior domains. Ann. Inst. H. Poincaré Anal. Non Linéaire 28(2), 303-313 (2011)

[30] Kozono, H., Ogawa, T.: Two-dimensional Navier-Stokes flow in unbounded domains. Math. Ann. 297, 1-31 (1993)

[31] Kozono, H., Yamazaki, M.: Local and global unique solvability of the Navier-Stokes exterior problem with Cauchy data in the space $L^{n, \infty}$. Houston J. Math. 21, 755-799 (1995)

[32] Kozono, H., Yamazaki, M.: On a larger class of stable solutions to the Navier-Stokes equations in exterior domains. Math. Z. 228, 751-785 (1998)

[33] Kozono, H., Yamazaki, M.: Exterior problem for the stationary Navier-Stokes equations in the Lorentz space. Math. Ann. 310, 279-305 (1998)

[34] Ladyzhenskaya, O. A.: The Mathematical Theory of Viscous Incompressible Flow. Gordon and Breach, New York (1969)

[35] Maekawa, Y.: On asymptotic stability of global solutions in the weak $L^{2}$ space for the two-dimensional Navier-Stokes equations. To appear in Analysis: International mathematical journal of analysis and its applications.

[36] Maremonti, P., Solonnikov, V.: On nonstationary Stokes problem in exterior domains. Ann. Scuola Norm. Sup. Pisa Cl. Sci. 24, 395-449 (1997)

[37] Masuda, K.: Weak solutions of Navier-Stokes equations. Tohoku Math. J. 36, 623-646 (1984) 
[38] Nakatsuka, T.: On uniqueness of symmetric Navier-Stokes flows around a body in the plane. Adv. Differential Equations. 20, 193-212 (2015)

[39] Novotny, A., Padula, M.: Note on decay of solutions of steady Navier-Stokes equations in 3-D exterior domains. Differential and Integral Equations. 8, 1833-1842 (1995)

[40] Pileckas, K., Russo, R.: On the existence of vanishing at infinity symmetric solutions to the plane stationary exterior Navier-Stokes problem. Math. Ann. 352, 643-658 (2012)

[41] Russo, A.: A note on the exterior two-dimensional steady-state Navier-Stokes problem. J. Math. Fluid Mech. 11, 407-414 (2009)

[42] Silvestre, A. L.: On the Existence of Steady Flows of a Navier-Stokes Liquid Around a Moving Rigid Body. Math. Meth. Appl. Sci. 27, 1399-1409 (2004)

[43] Simader, C. G., Sohr, H.: A new approach to the Helmholtz decomposition and the Neumann problem in $L^{q}$-spaces for bounded and exterior domains. In: Mathematical Problems Relating to the Navier-Stokes Equations, pp. 1-35. Ser. Adv. Math. Appl. Sci., 11. World Sci. Publishing, River Edge (1992)

[44] Sohr, H.: The Navier-Stokes equations. An elementary functional analytic approach. Birkhäuser Advanced Texts: Basler Lehrbücher. Birkhäuser Verlag, Basel (2001)

[45] Yamazaki, M.: The Navier-Stokes equations in the weak- $L^{n}$ space with timedependent external force. Math. Ann. 317, 635-675 (2000)

[46] Yamazaki, M.: Unique existence of stationary solutions to the two-dimensional Navier-Stokes equations on exterior domains. Mathematical Analysis on the NavierStokes Equations and Related Topics, Past and Future - In memory of Professor Tetsuro Miyakawa, Gakuto International Series in Mathematical Sciences and Applications, Vol. 35, pp. 220-241. Gakkōtosho, Tokyo (2011) 